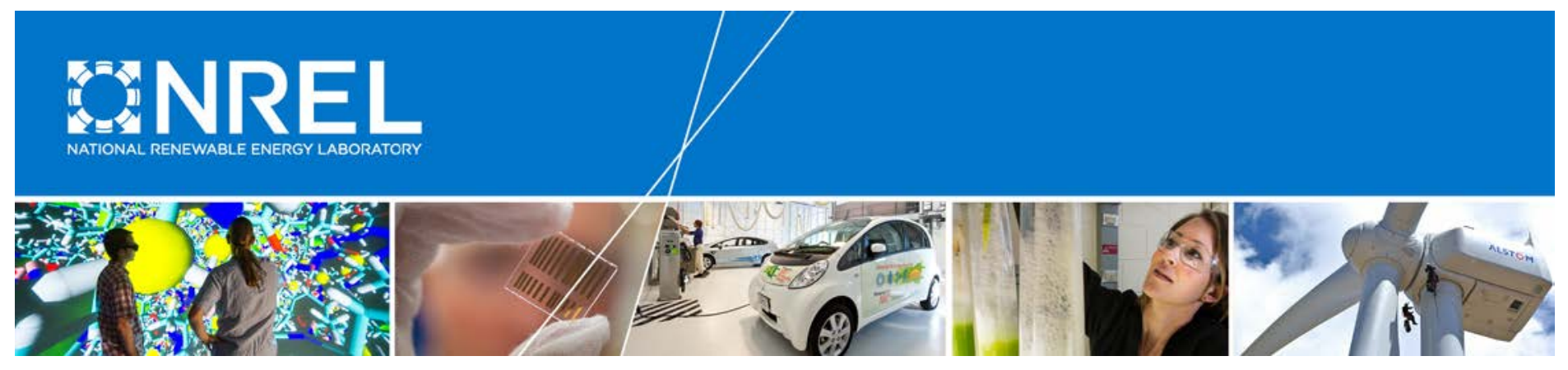

\title{
Compendium of Experimental Cetane Numbers
}

J. Yanowitz

Ecoengineering

M.A. Ratcliff, R.L. McCormick, and J.D. Taylor National Renewable Energy Laboratory

\section{M.J. Murphy}

Battelle

Based on the Compendium of Experimental Cetane Numbers, NREL/TP-5400-61693, August 2014

NREL is a national laboratory of the U.S. Department of Energy Office of Energy Efficiency \& Renewable Energy Operated by the Alliance for Sustainable Energy, LLC

This report is available at no cost from the National Renewable Energy Laboratory (NREL) at www.nrel.gov/publications.

Technical Report

NREL/TP-5400-67585

February 2017

Contract No. DE-AC36-08G028308 


\section{Compendium of Experimental Cetane Numbers}

J. Yanowitz

Ecoengineering

M.A. Ratcliff, R.L. McCormick, and J.D. Taylor National Renewable Energy Laboratory

M.J. Murphy

Battelle

Prepared under Task No. VTP2.9600

NREL is a national laboratory of the U.S. Department of Energy Office of Energy Efficiency \& Renewable Energy Operated by the Alliance for Sustainable Energy, LLC

This report is available at no cost from the National Renewable Energy Laboratory (NREL) at www.nrel.gov/publications.

National Renewable Energy Laboratory 15013 Denver West Parkway Golden, CO 80401

303-275-3000 • www.nrel.gov

\section{Technical Report}

NREL/TP-5400-67585

February 2017

Contract No. DE-AC36-08GO28308 


\section{NOTICE}

This report was prepared as an account of work sponsored by an agency of the United States government. Neither the United States government nor any agency thereof, nor any of their employees, makes any warranty, express or implied, or assumes any legal liability or responsibility for the accuracy, completeness, or usefulness of any information, apparatus, product, or process disclosed, or represents that its use would not infringe privately owned rights. Reference herein to any specific commercial product, process, or service by trade name, trademark, manufacturer, or otherwise does not necessarily constitute or imply its endorsement, recommendation, or favoring by the United States government or any agency thereof. The views and opinions of authors expressed herein do not necessarily state or reflect those of the United States government or any agency thereof.

This report is available at no cost from the National Renewable Energy Laboratory (NREL) at www.nrel.gov/publications.

Available electronically at SciTech Connect http:/www.osti.gov/scitech

Available for a processing fee to U.S. Department of Energy and its contractors, in paper, from:

U.S. Department of Energy

Office of Scientific and Technical Information

P.O. Box 62

Oak Ridge, TN 37831-0062

OSTI http://www.osti.gov

Phone: 865.576.8401

Fax: 865.576.5728

Email: reports@osti.gov

Available for sale to the public, in paper, from:

U.S. Department of Commerce

National Technical Information Service

5301 Shawnee Road

Alexandria, VA 22312

NTIS http://www.ntis.gov

Phone: 800.553 .6847 or 703.605 .6000

Fax: 703.605.6900

Email: orders@ntis.gov 


\section{Acknowledgments}

The authors would like to acknowledge the assistance of Jon Luecke and Lisa Fouts in using the ignition quality tester at the National Renewable Energy Laboratory and the assistance of Andre Boehman (Department of Mechanical Engineering, University of Michigan), Mac Haas (Department of Mechanical and Aerospace Engineering, Princeton University), Anthony Marchese (Department of Mechanical Engineering, Colorado State University), Jim Anderson, (Ford Motor Company) in locating cetane number data.

Joshua D. Taylor is currently with SABIC, Sugarland, Texas. 


\section{List of Acronyms}

ASTM

CAD

CFR

$\mathrm{CN}$

CVCC

DCN

FIT

HMN

IQT

NREL

PRF
ASTM International

crank angle degree

Cooperative Fuel Research

cetane number

constant-volume combustion chamber

derived cetane number

Fuel Ignition Tester

2,2,4,4,6,8,8-heptamethylnonane

Ignition Quality Tester

National Renewable Energy Laboratory

primary reference fuel 


\section{Executive Summary}

This report is an updated version of the 2014 Compendium of Experimental Cetane Number Data and presents a compilation of measured cetane numbers for pure chemical compounds. It includes all available single-compound cetane number data found in the scientific literature up until December 2016 as well as a number of previously unpublished values, most measured over the past decade at the National Renewable Energy Laboratory. This version of the compendium contains cetane values for 497 pure compounds, including 204 hydrocarbons and 293 oxygenates. One hundred seventy-five individual measurements are new to this version of the compendium, all of them collected using ASTM Method D6890, which utilizes an Ignition Quality Tester (IQT), a type of constant-volume combustion chamber. For many compounds, numerous measurements are included, often collected by different researchers using different methods. The text of this document is unchanged from the 2014 version, except for the numbers of compounds in Section 3.1; the appendices; Table 1, Primary Cetane Number Data Sources; and Table 2, Number of Measurements Included in Compendium.

Cetane number is a relative ranking of a fuel's autoignition characteristics for use in compression ignition engines. It is based on the amount of time between fuel injection and ignition, also known as ignition delay. The cetane number is typically measured either in a single-cylinder engine or a constant-volume combustion chamber. Values in the 2004 compendium derived from octane numbers have been removed and replaced with a brief analysis of the correlation between cetane numbers and octane numbers. The discussion on the accuracy and precision of the most commonly used methods for measuring cetane number has been expanded, and the data have been annotated extensively to provide additional information that will help the reader judge the relative reliability of individual results. 


\section{Table of Contents}

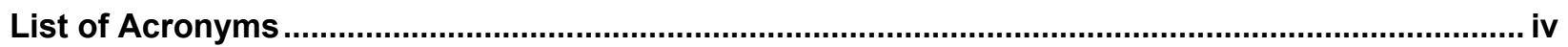

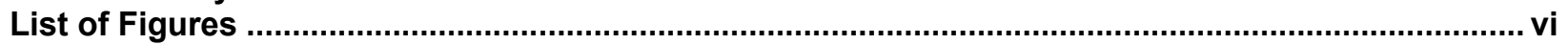

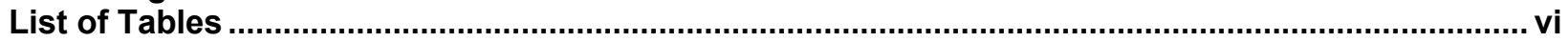

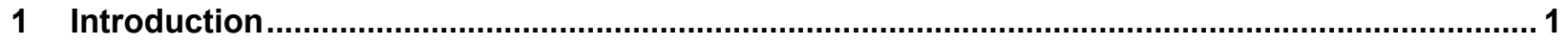

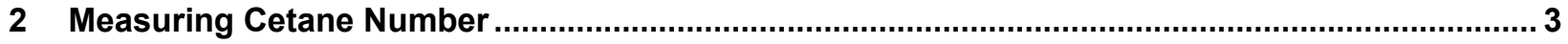

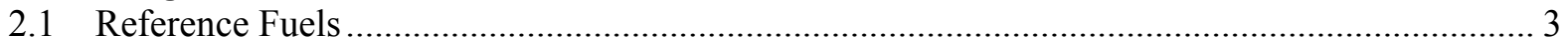

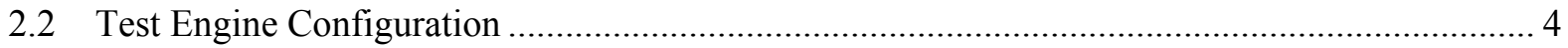

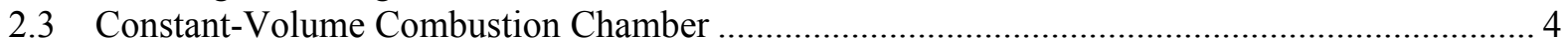

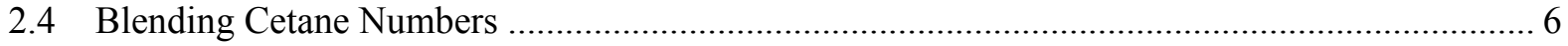

3 Cetane Number Data Quality

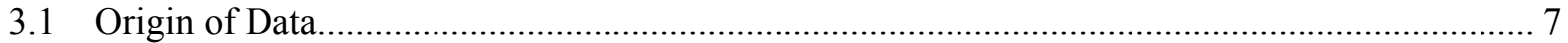

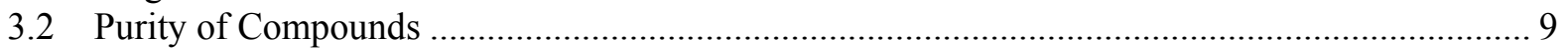

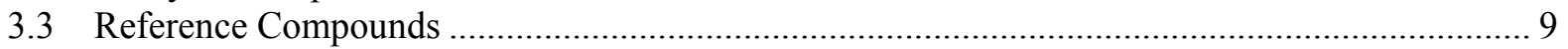

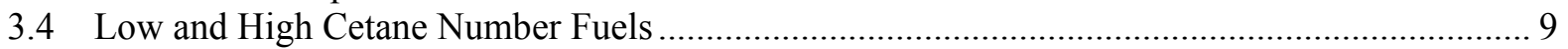

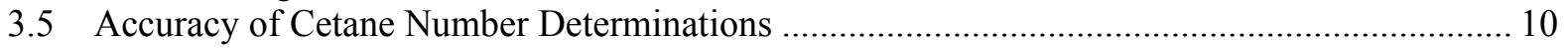

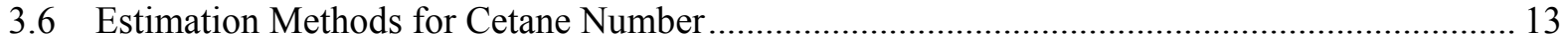

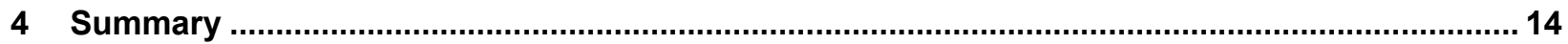

Appendix A. Compendium of Experimental Cetane Number Data ................................................... 15

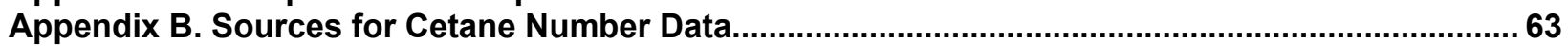

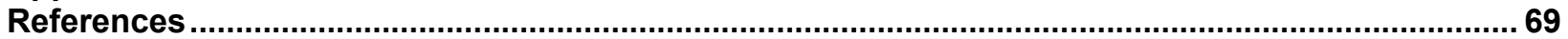

\section{List of Figures}

Figure 1. Structure of 1-hexadecene (cetene or ketene) ...................................................................... 3

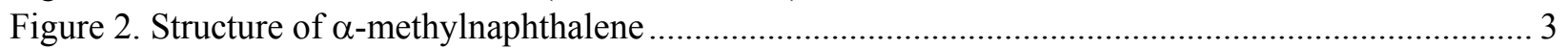

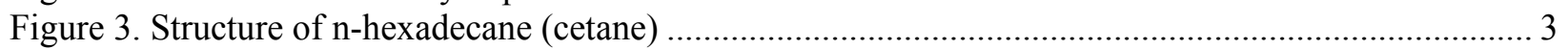

Figure 4. Structure of $2,2,4,4,6,8,8$-heptamethylnonane ................................................................ 4

Figure 5. ASTM repeatability of ASTM D613, ASTM D6890, and ASTM D7170 .............................. 11

Figure 6. ASTM reproducibility of ASTM D613, ASTM D6890, and ASTM D7170 ........................... 11

Figure 7. Comparison of different methods for measuring cetane number ......................................... 12

Figure 8. Correlation between cetane number and research octane number suggests cetane number for both oxygenates and hydrocarbons can be roughly estimated as $56-(0.39 \mathrm{x}$ research octane number).

\section{List of Tables}

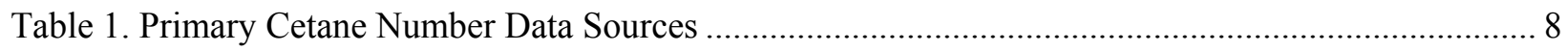

Table 2. Number of Measurements Included in Compendium ........................................................... 10

Table 3. Average Absolute Difference between DCN by IQT and Values Measured by Other Methods.. 13 


\section{Introduction}

Cetane number $(\mathrm{CN})$ is a relative measure of the time delay between the injection of fuel into the chamber and the start of combustion. Fuels for compression ignition engines must autoignite readily. If ignition does not occur promptly when the fuel is injected into the cylinder, premixed fuel and air accumulate such that when ignition does occur, the rate of burning is too rapid. The rapid burning produces high pressure rise rates that can result in engine knock that decreases efficiency and can damage the engine. Thus, the ability to rate the ignition quality of compression ignition fuels is important to diesel fuel formulation. Without adequate fuel ignition quality (a high enough cetane number), the engine will start with difficulty and run poorly.

This report presents the results of an exhaustive literature search for available experimental cetane number data for pure compounds as of December 2016 and briefly describes the process of compression ignition, the methods for measuring diesel fuel ignition quality, and the sources of uncertainty in cetane number data.

The authors anticipate that a revised and updated version of this report will be prepared every few years. Researchers are encouraged to share additional cetane number data on pure compounds as they are published for inclusion in future editions.

A compression ignition engine uses the heat of compression to ignite the fuel in the cylinders of the engine. Each cylinder is filled with air through the intake valve. The intake valve is then shut, and the motion of the piston reduces the volume of air, compressing and heating the air. At roughly the point of maximum compression, liquid fuel is injected into the cylinder through a nozzle. The fuel forms a spray of droplets that vaporize, mix with hot air, and then ignite. As the fuel burns, the gas in the cylinder heats and expands, driving the piston.

Combustion occurs in the gas phase. Thus, for a liquid fuel, the first steps toward ignition involve transitioning from a liquid to a gas. The time required for this transition is the "physical delay" in ignition and includes the amount of time required for a droplet of fuel to heat, vaporize, and mix with hot air in the cylinder.

The physical delay is influenced by $[1,2]$ :

- Density and temperature of the air in the cylinder

- Velocity and turbulence of the air

- Atomization, penetration, and shape of the spray

- The properties of the fuel, including:

$\circ$ Density

○ Viscosity

○ Surface tension

- Specific heat

$\circ$ Enthalpy of vaporization 


\section{○ Vapor pressure \\ - Vapor diffusivity.}

Following the physical processes of vaporization and air mixing, a sequence of chemical reactions occurs in which the gas-phase fuel reacts with oxygen. In order to ignite, the fuel must be heated to a temperature sufficient for some of the weaker bonds within the molecules to break and form radicals. The finite rate of these radical-forming oxidation reactions is responsible for the chemical delay in compression ignition. Once a sufficient concentration of free radicals is reached, rapid oxidation occurs (ignition).

Early work by Yu et al. [3] and more recent work focused on the IQT by Bogin and coworkers [4] were able to separate the effects of physical and chemical ignition delay. While large gradients in stoichiometry and temperature occur within the IQT at DCN conditions, the chemical ignition delay is a dominant factor over the physical ignition delay for determining the measured ignition delay time and correlated DCN measurement. 


\section{Measuring Cetane Number}

The earliest evaluations of diesel fuels were most likely audible; some fuels caused the engine to operate more smoothly than others. In time, quantitative scales were developed to more readily compare fuels.

\subsection{Reference Fuels}

During the 1930s, Boerlage and Broeze [5] of the Delft Laboratory in the Netherlands sought a procedure to determine the ignition quality of diesel fuel that was similar to the octane rating method for gasoline using two reference hydrocarbon fuels: 1-hexadecene and $\alpha$-methylnaphthalene. The first reference fuel, 1-hexadecene, also known as cetene or ketene, has a long, straight chain structure, as shown in Figure 1, and oxidizes relatively easily. This fuel was assigned a cetene (ketene) number of 100.

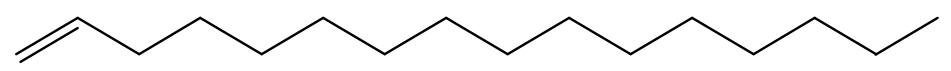

Figure 1. Structure of 1-hexadecene (cetene or ketene)

The second reference fuel, $\alpha$-methylnaphthalene, also known as 1-methylnaphthalene, has two aromatic rings, as shown in Figure 2, and is highly resistant to autoignition. This fuel was assigned a cetene number of 0 . The cetene number of a fuel was deemed to be the percent (by mass) of cetene in a blend of cetene and $\alpha$-methylnaphthalene that gave the same ignition performance as the fuel under test.<smiles>Cc1cccc2ccccc12</smiles>

Figure 2. Structure of $\alpha$-methylnaphthalene

Researchers in the United States found it was difficult to ensure all of the 1-hexadecene (cetene) had the double bond in the same position. Moreover, 1-hexadecene was prone to oxidation during storage. Because of the difficulty of preparing pure cetene, n-hexadecane (cetane) replaced cetene as the primary reference fuel (PRF) and was assigned a cetane rating of 100 . The cetane number of a PRF blend was defined as:

$$
\mathrm{CN}=\% \text { by volume } n \text {-hexadecane }+\% \text { by volume } \alpha \text {-methylnaphthalene }
$$

The structure of n-hexadecane (cetane) is shown in Figure 3.

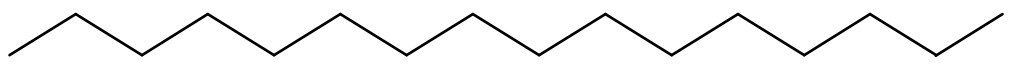

Figure 3. Structure of $\mathbf{n}$-hexadecane (cetane)

Comparison of the two ratings showed the following approximate relationship between cetane rating and cetene (ketene) rating [6]:

$$
\text { Cetane Rating }=0.875 \times \text { Cetene Rating }
$$


Because of experimental difficulties in working with $\alpha$-methylnaphthalene, a suspected carcinogen with a foul odor, the reference fuel for the lower end of the cetane number scale was also changed. In this case the new reference fuel was 2,2,4,4,6,8,8-heptamethylnonane (HMN), shown in Figure 4, with an assigned cetane number of 15.

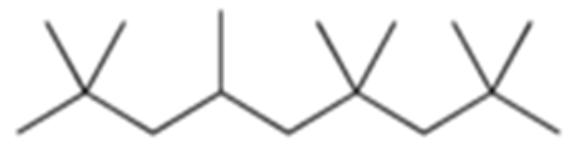

Figure 4. Structure of 2,2,4,4,6,8,8-heptamethylnonane

The ASTM International (ASTM) method for measuring cetane number is D613, Standard Test Method of Diesel Fuel Oil. The method was first published in 1941, and subsequently a key change was made, substituting $\mathrm{HMN}(\mathrm{CN}=15)$ for $\alpha$-methylnaphthalene $(\mathrm{CN}=0)$ as the PRF at the lower end of the scale.

The cetane number of a PRF blend is now defined as:

$$
\mathrm{CN}=\% \text { by volume } \mathrm{n} \text {-hexadecane }+0.15 \times(\% \text { by volume } \mathrm{HMN})
$$

\subsection{Test Engine Configuration}

Originally, the cetene rating scale called for finding the lowest compression ratio that would produce autoignition. This proved to be imprecise. Subsequently, Boerlage and Broeze [5] proposed a method using a single-cylinder diesel Cooperative Fuel Research (CFR) engine made by Waukesha. Testing was done by injecting the fuel 10 crank angle degrees (CAD) before top dead center and adjusting a plunger in the prechamber to obtain ignition at $1 \mathrm{CAD}$ after top dead center, for an ignition delay of $11 \mathrm{CAD}$. This method formed the basis for developing the ASTM D613 standard for cetane number, although the final method included a modified standard ignition delay of $13 \mathrm{CAD}$.

In the ASTM D613 test, the cetane number of a diesel fuel is determined by comparing its ignition delay in the standard CFR test engine with those for blends of reference fuels of known cetane number. The compression ratio is varied by adjusting a calibrated hand wheel to obtain the same ignition delay for the sample and for each of two bracketing reference fuels, permitting interpolation of cetane number in terms of the hand wheel readings. The ISO 5165 method, the international counterpart to ASTM D613, is essentially the same test using the same engine.

In Europe, a similar method, DIN 51773, uses a standard BASF engine for determining cetane number. Both the CFR and BASF methods vary effective compression ratios, thus varying the available energy to start combustion, but in different ways. The Waukesha CFR engine varies the physical volume of the combustion chamber, thereby changing the compression ratio of the engine and changing the amount of energy available for initiating combustion. The BASF engine, however, varies the amount of air allowed to enter the cylinder while maintaining the same physical compression ratio.

\subsection{Constant-Volume Combustion Chamber}

The first attempts at quantitatively measuring compression ignition fuel quality involved the use of a bench-top apparatus. Falk began to measure the compression autoignition temperature of 
fuels in 1906 [7]; however, Falk did not measure the variation of cylinder pressure with time and so did not notice any ignition delay. Later, in 1914, Dixon and Howard [8] recognized the existence of an ignition delay period.

Mullins [9] reports that in 1932, Helmore and Code-Holland developed a constant-volume apparatus specifically for testing diesel fuels. The apparatus was claimed to simulate diesel engine conditions, but it operated at atmospheric pressure and could only measure ignition delays longer than 200 milliseconds. Nonetheless, they observed some agreement with the behavior of test fuels in a diesel engine.

Work by Hurn and Hughes [10] of the U.S. Bureau of Mines led to the development of a constant-volume diesel fuel test apparatus consisting of a pressurized and heated reaction chamber with a single-shot fuel injector, along with instrumentation to measure the time delay between injection and ignition. With this apparatus, the effect of cetane number on ignition delay was observed, but the researchers did not develop a specific cetane number correlation.

More recently, studies of constant-volume combustion were undertaken by Ryan and coworkers at Southwest Research Institute with the goal of developing a new method for rating the ignition delay of diesel fuels $[11,12]$. These studies resulted in the development of an ignition quality tester (IQT) in which a sample of fuel is injected into a heated, constant-volume combustion chamber (CVCC). Initially the pressure decreases due to cooling from fuel evaporation, but rises as combustion begins reaching the initial pressure at the pressure recovery point. The ignition delay is measured as the time delay between the beginning of injector needle lift and the chamber pressure recovery point. A correlation was developed between the observed ignition delay and the cetane number. The method has been refined, validated, and commercialized by Advanced Engine Technology, Ltd. [13, 14, 15] and has been approved by ASTM as Standard D6890. A similar method using the Waukesha Fuel Ignition Tester (FIT) has been formalized in ASTM Standard D7170. Most recently, Herzog by PAC has introduced the Cetane ID 510 with associated ASTM Standard D7668. Prior to finalization of these ASTM standards, cetane numbers were obtained using similar equipment (in some cases these CVCC prototypes), although the methodology may not have met all of the requirements of the ASTM standards.

CVCC methods use much smaller sample volumes than the engine test procedure (on the order of 100 milliliters versus 1 liter for the engine test), which can be significant for expensive and difficult-to-produce pure compounds. The CVCC methods can also be completed in much shorter time frame (20 minutes versus a few hours). Moreover, because the reproducibility errors (discussed in more detail in Section 3.5) are lower for the IQT than those found for the CFR engine, it is generally considered the better method for measuring cetane number at this time. Most new data reported in this version of the compendium are IQT values.

Because the cetane number determined by CVCC methods is not measured in the actual CFR engine, which is the defined source of cetane number values, the values that result from this approach are known as the derived cetane number (DCN). The CVCC methods are calibrated to the CFR engine using hydrocarbon compounds. In practice, correlation between the methods has been quite good for the mid-range cetane number distillate fuels for which both the CVCC and the CFR methods are most commonly used. However, because each method measures ignition delay under different conditions (pressure, temperature, and stoichiometry), it is possible that the 
effects of these different operating conditions may not result in the same correlation for all compounds $[14,16]$. For ASTM standard compliance, the D613 CFR engine method remains the referee method.

\subsection{Blending Cetane Numbers}

In some cases, cetane number data were not available for the pure compound, but were available for blends of a known volume of the pure compound in diesel fuel of known cetane number. In such cases, it is possible to compute a blending cetane number. However, the typical methodology for developing blending cetane numbers results in an amplification of uncertainty. Assuming the cetane number of the blend is a linear combination of the cetane numbers of the components, we expect for a $10 \%$ blend:

$$
\text { Blend } \mathrm{CN}=(0.9) \times(\text { Base Fuel } \mathrm{CN})+(0.1) \times(\text { Test Fuel } \mathrm{CN})
$$

or

$$
\text { Test Fuel CN }=[(\text { Blend CN })-(0.9) \times(\text { Base Fuel CN })] / 0.1
$$

However, any measurement error in the blend $\mathrm{CN}$ will be multiplied by the inverse of the blend level when the test fuel $\mathrm{CN}$ is calculated (e.g., a 20\% blend results in a five-fold increase in the magnitude of the error; see sample calculation in box). Inasmuch as the base fuel cetane number measurement is also subject to measurement error and that many studies report cetane number measurement errors greater than $0.5 \mathrm{CN}$, the possibility exists for large errors in the reported blending cetane numbers.

A separate issue is whether or not the cetane number of a blend is indeed a linear combination of the cetane numbers of the components. Although the CFR cetane number scale is based on the linear blending values of the two PRFs, n-hexadecane and HMN, there is evidence that the linear assumption is not correct for all blends. For example, in their study of the cetane numbers of carboxylic esters, Serdari et al. [17] find the cetane numbers of methyl oleate blends appear to behave linearly, while those of ethyl laurate blends appear to behave non-linearly. Nonetheless, blending cetane number data may be the only information available for some pure components. In the compendium of cetane numbers, blending cetane numbers are included, but are flagged to warn users of the possible uncertainties. However, for this reason, it is recommended that blend values be considered a rough approximation of the actual cetane number. If better information is available from tests of the compound in pure form the use of those values is preferred.

Example of Amplification of Measurement Error in Blend Fuel Calculations:

If the base fuel $\mathrm{CN}$ is 50 and the test fuel $\mathrm{CN}$ is 10 , a $10 \%$ blend of test fuel in the base fuel, assuming $\mathrm{CN}$ is a linear combination of the components (using Eq. 3), the true $\mathrm{CN}$ value of the blend is:

$$
\text { Blend } \mathrm{CN}=(0.9) \times(50)+(0.1) \times(10)=46
$$

If the measured $\mathrm{CN}$ is 47 , only $1 \mathrm{CN}$ point larger than the true value, then, using Eq. 4 , the calculated value of the test fuel $\mathrm{CN}$ would be:

Calculated test fuel $\mathrm{CN}=[(47)-(0.9) \times(50)] / 0.1=20$, a $\mathrm{CN}$ value that is $10 \mathrm{CN}$ points larger than the true value. 


\section{Cetane Number Data Quality}

\subsection{Origin of Data}

Referenced sources and technical papers were sought in which cetane number data were presented for pure compounds. Based on this search conducted first in 2004 and then again in 2013, a summary of all available cetane number data for pure compounds was developed and is included in Appendix A. The results include the measured cetane numbers of 496 pure compounds, including 204 hydrocarbons and 292 oxygenates. The total number of measurements is 760 , from 77 different sources, which include many compounds for which there are values available from more than one source, and many measurements that appear to have been cited in more than one source. Where that was apparent, those data points have been combined in the appendix. All sources for the information are cited in Appendix B.

There are several references that have been used as "handbooks" of cetane number data. One recent source of cetane number data is the 1999 book Fuels and Engines by J.C. Guibet [18], which lists cetane number data for approximately 100 pure compounds. However, these data are, in fact, the same cetane number values ${ }^{*}$ for the same compounds that are found in Technical Data on Fuels by Rose and Cooper, published in 1977 [19].

The data in Rose and Cooper are not individually referenced, but are stated to be taken mainly from the 1948 U.S. Bureau of Mines Information Circular 7474 by Puckett and Caudle that contains cetane number data for 98 compounds [20]. The Bureau of Mines circular contains references to the source of each cetane number value. From these references we learn that cetane numbers for most of the compounds in Circular 7474 are quoted from work done by Petrov in Russia from 1938 to 1946 using a combustion bomb apparatus called the Nuemann bomb to measure ignition delay, then using a correlation between ignition delay and cetene number. A 1938 correlation between the cetene number scale and the cetane number scale, cited in Section 2.1, was applied to "convert" Petrov's results to a cetane number value. Another recent source, Chevron's Diesel Fuels Technical Review [21] lists cetane numbers for 21 compounds. No references are given for these data, but there are indications that they too are derived from the Russian work quoted by Puckett and Caudle. ${ }^{\dagger}$ Multiple identical values of the same cetane number were reduced to a single line in the appendix and attributed to all of the sources in which they appear and which we were able to obtain. Thus, the many data points attributed to any one of these sources were likely derived from World War II-era ignition delay measurements and the successive application of two correlations (from ignition delay to cetene number and from cetene number to cetane number).

Serdari et al. [16] present cetane numbers for 64 esters measured in blends of only $5 \%$ to $7 \%$, and they estimate their precision at \pm 7 to 10 cetane units. Knothe, Matheaus, and Ryan [22] present cetane number data for 29 fatty acid esters. In an earlier paper, Knothe, Bagby, and Ryan [23] list cetane numbers derived from an ignition delay procedure for 21 esters, alcohols, and triglycerides. Freedman et al. [24] present data for 20 esters, alcohols, and triglycerides. There is

\footnotetext{
${ }^{*}$ There is a transcription error for 2-methyl-4-isobutyl-4-phenylundecane: instead of the cetane number of 18 found in Rose and Cooper, Guibet lists a cetane number for this compound of 38.

" A "5" for " 8 " transcription error for the cetane number of 3-ethyldecane that occurred going from a 1946 review paper prepared by Petrov to the Bureau of Mines report and then carried through all the later references supports this hypothesis.
} 
substantial overlap between these sources in terms of compounds studied; where there is overlap, the agreement is often poor. Most data for fatty acid esters are from a CVCC-based ignition delay apparatus. Only a few values are reported to be from the D613 engine test, and in those cases the experimental procedure is poorly documented considering that in some cases the esters tested are solids at normal ambient temperatures. It is not clear whether the entire apparatus was heated above the melting point or whether the values reported are actual blending cetane numbers with an unspecified diesel fuel.

In the original 2004 version of the compendium, the National Renewable Energy Laboratory (NREL) was the source of 26 data points, all measured on the IQT. Additional compounds measured on the NREL IQT bring the total number of compounds measured at this laboratory to 108, including a range of alkanes, iso-alkanes, cyclo-alkanes, alkenes, aromatics, esters, alcohols, and ethers using ASTM D6890 (IQT). Some of these have been published in scientific literature, but many are published here for the first time.

This compendium also includes individual values collected in small numbers from a variety of scientific papers. Table 1 lists the most important sources of data in this compendium. These seven sources comprise approximately $70 \%$ of the data in this version of the compendium. As noted above, there is considerable overlap in the measurements listed in References 3 and 4 in Table 1.

Table 1. Primary Cetane Number Data Sources

\begin{tabular}{|c|l|c|}
\hline $\begin{array}{c}\text { Reference } \\
\text { Number }\end{array}$ & \multicolumn{1}{|c|}{ Reference } & $\begin{array}{c}\text { Number of } \\
\text { Measurements } \\
\text { Included in } \\
\text { Compendium }\end{array}$ \\
\hline 41 & NREL IQT data & 108 \\
\hline 3 & $\begin{array}{l}\text { Puckett, A.D.; Caudle, B.H. (1948). Ignition Qualities of } \\
\text { Hydrocarbons in the Diesel Fuel Boiling Range. Bureau Mines } \\
\text { Information Circular 7474. }\end{array}$ & 98 \\
\hline 4 & $\begin{array}{l}\text { Rose, J.W.; Cooper, J.R. (1977). "Detonation of Liquid Fuels." In } \\
\text { Technical Data on Fuel. }\end{array}$ & 89 \\
\hline 76 & $\begin{array}{l}\text { Dahmen, M.; Marquardt, W. (2015) "A Novel Group Contribution } \\
\text { Method for the Prediction of the Derived Cetane Number of } \\
\text { Oxygenated Hydrocarbons." Energy Fuels 29 (9); pp. 5781- } \\
\text { 5801. }\end{array}$ & 79 \\
\hline 32 & $\begin{array}{l}\text { Serdari, A.; Lois, E.; Stournas, S. (1999). "Impact of Esters of } \\
\text { Mono- and Dicarboxylic Acids on Diesel Fuel Quality." Ind. Eng. } \\
\text { Chem. Res. (38); p. 3543. }\end{array}$ & 64 \\
\hline 1 & $\begin{array}{l}\text { Olson, D.R.; Meckel, N.T.; Quillian, R.D. (1960). “Combustion } \\
\text { Characteristics of Compression Ignition Engine Fuel } \\
\text { Components." SAE paper 600112. }\end{array}$ & 40 \\
\hline 33 & $\begin{array}{l}\text { Knothe, G.; Matheaus, A.C.; Ryan III, T.W. (2003). "Cetane } \\
\text { Numbers of Branched and Straight-Chain Fatty Esters } \\
\text { Determined in an Ignition Quality Tester." Fuel (82); p. 971. }\end{array}$ & 28 \\
\hline
\end{tabular}




\subsection{Purity of Compounds}

There are few data on the purity of the compounds that were used for cetane number determinations. Because of the relatively large sample size required for the ASTM D613 engine test, assembling samples of high purity levels is challenging and potentially very expensive. Older data typically did not include information on purity levels or the identity of possible impurities.

Peroxides (compounds with an R-O-O-R linkage), in particular, have been found in many compounds at levels sufficient to affect the measured cetane number [25]. Because most peroxides are extremely reactive, they have long been known to be effective additives for improving the cetane number of diesel fuels [26]. Peroxides can be formed by the auto-oxidation of hydrocarbons in storage. Even the n-hexadecane (cetane) diesel reference fuel can contain peroxides that affect the results of cetane number determinations.

ASTM D6890 requires all samples be filtered through a 3-5-micron filter to remove particulates and that the sample be at room temperature $\left(18^{\circ} \mathrm{C}-32^{\circ} \mathrm{C}\right)$. However, because calibrating the IQT with the low cetane check fuel (methylcyclohexane) was so frequently problematic, Advanced Engine Technology (the owner of the IQT technology) investigated sample purity to determine the source of the contamination [27].The study found that filtering methylcyclohexane through a $\left(250^{\circ} \mathrm{C}\right.$ treated) silica gel column improved the ignition delay reproducibility. Although specific contaminants were not identified in this study, silica gel is polar and will adsorb and remove polar contaminants like water or oxygenates. To ensure high purity and the removal of peroxides, modern standard practice for hydrocarbons and non-polar oxygenates employs column chromatography on samples prior to testing. All samples should be evaluated for their purity prior to testing.

\subsection{Reference Compounds}

The accuracy of the definition of HMN as a PRF with a cetane number of 15 was called into question as long ago as the 1974 study by Bowden et al. of Southwest Research Institute [28]. He reported that, using $\alpha$-methylnaphthalene and hexadecane as reference fuels, the cetane number of HMN was found to be only 12.2 and not 15 , as is customarily assigned when it is used as a reference fuel. IQT measurements of HMN at NREL produce a consistent DCN value of 15.1 .

For fuels with cetane numbers typical of diesel fuels in the United States (in the low 40s), this would make a difference of slightly more than one cetane number unit when comparing data from cetane number scales based on the older and newer reference compounds.

\subsection{Low and High Cetane Number Fuels}

Currently there is no accepted methodology for extending the cetane number scale to cetane numbers less than zero or greater than 100 . While this can be done using blending cetane numbers, this approach is not rigorous and in some cases leads to very different results with different base fuels. Many samples tested using the IQT included in this compendium fall outside the limits of ASTM D6890 (33 to 64 DCN). Testing has not been undertaken to ensure repeatability and reproducibility of samples outside this range, nor have comparisons been conducted to ensure that the DCNs correspond to $\mathrm{CN}$ as measured by D613. 


\subsection{Accuracy of Cetane Number Determinations}

Table 2 lists the number of data points included in this compendium from each type of measurement method. Upon review of the original sources, a number of measurements have been reclassified from the original compendium. These changes have been included in the notes in Appendix A.

Blend measurements, by their nature, are less accurate than the underlying measurement method. Unknown methods and other ignition delay methods suffer from varying methodology and uncertain correlation, as well as generally being older and thus potentially made using samples of lesser purity. Thus, data collected using ASTM D613 (CFR), D6890 (IQT), and D7170 (FIT) should be considered the most trustworthy because the methods themselves are well documented, consistently implemented, and correlated with each other.

Table 2. Number of Measurements Included in Compendium

\begin{tabular}{|l|l|l|l|l|l|l|l|}
\hline & $\begin{array}{c}\text { ASTM } \\
\text { Method } \\
\text { D613 } \\
\text { (CFR) }\end{array}$ & $\begin{array}{c}\text { ASTM } \\
\text { Method } \\
\text { D6890 } \\
\text { (IQT) }\end{array}$ & $\begin{array}{c}\text { ASTM } \\
\text { Method } \\
\text { D7170 } \\
\text { (FIT) }\end{array}$ & $\begin{array}{c}\text { Other } \\
\text { Ignition } \\
\text { Delay } \\
\text { Method }\end{array}$ & Blend & $\begin{array}{c}\text { Unknown } \\
\text { Method }\end{array}$ & Total \\
\hline $\begin{array}{l}\text { Measurements } \\
\text { in 2004 } \\
\text { Compendium }\end{array}$ & 3 & 16 & 0 & 76 & 85 & 142 & $\begin{array}{l}322 \text { measurements, } \\
296 \text { different } \\
\text { compounds }\end{array}$ \\
\hline $\begin{array}{l}\text { Measurements } \\
\text { in 2014 } \\
\text { Compendium }\end{array}$ & 58 & 128 & 6 & 70 & 135 & 189 & $\begin{array}{l}586 \text { measurements, } \\
387 \text { different } \\
\text { compounds }\end{array}$ \\
\hline $\begin{array}{l}\text { Measurements } \\
\text { in this } \\
\text { Compendium }\end{array}$ & 58 & 303 & 6 & 70 & 135 & 189 & $\begin{array}{l}761 \text { measurements, } \\
497 \text { different } \\
\text { compounds }\end{array}$ \\
\hline
\end{tabular}

In instances where cetane number data are available from more than one source, there is often poor agreement as shown in the graph below; differences of up to 15 cetane numbers are not uncommon in this compendium. The current ASTM methods D613, D6890, and D7170 (the CFR engine, IQT, and FIT procedures, respectively) list reproducibility and repeatability limits for the accepted range of the specification. Those values are shown in Figures 5 and 6. ASTM defines reproducibility as the difference between two test results on identical samples, but obtained by different operators in different laboratories, that would be exceeded only one case in 20. In other words, for D613 tests on a cetane number 48 fuel by multiple laboratories, $95 \%$ of the test results would be between 44.2 and 51.8. Repeatability is the difference between two test results on identical samples obtained by the same operator using the same apparatus, under constant operation conditions, on identical test materials that would be exceeded only one time in 20 . 


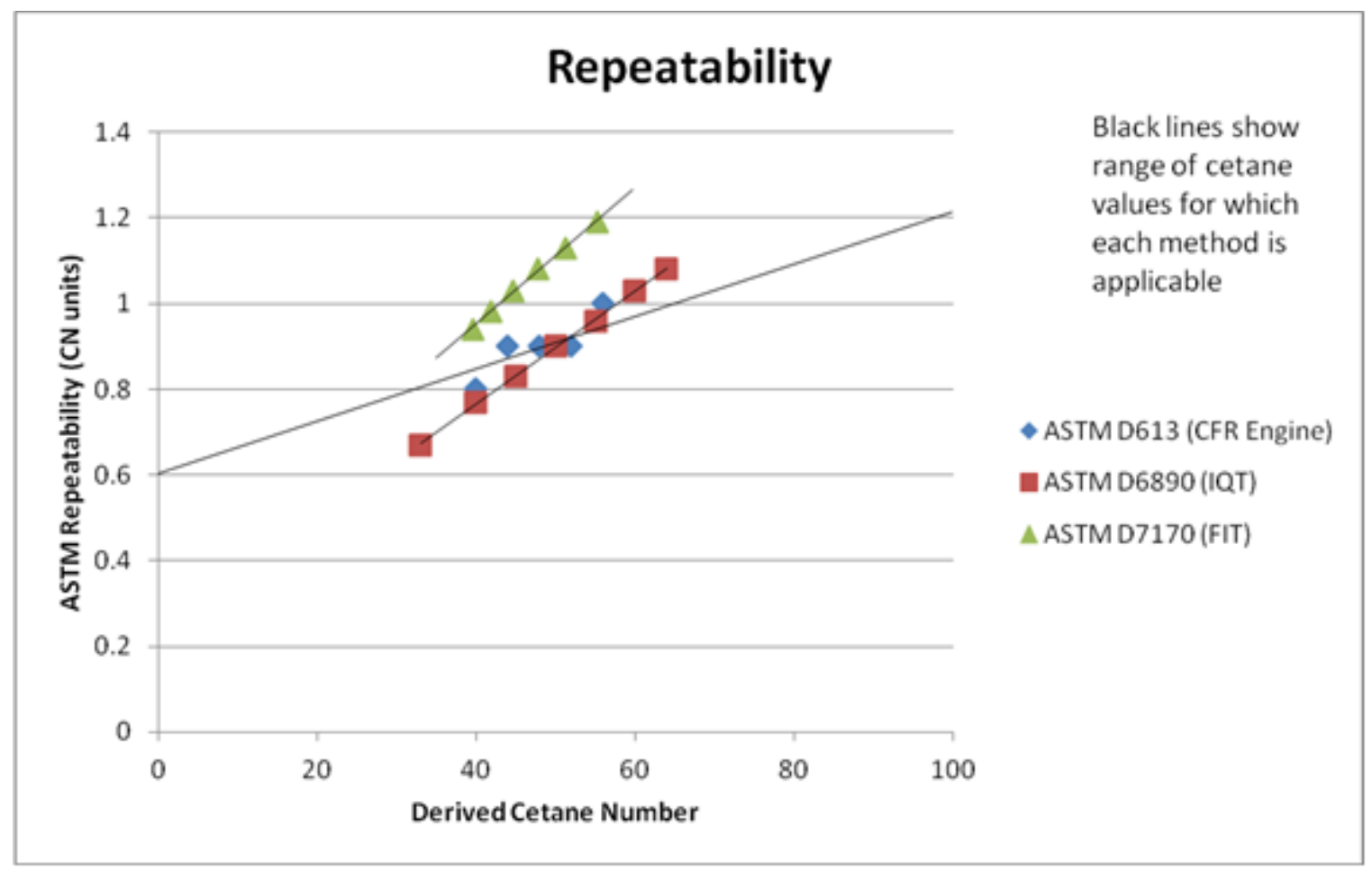

Figure 5. ASTM repeatability of ASTM D613, ASTM D6890, and ASTM D7170

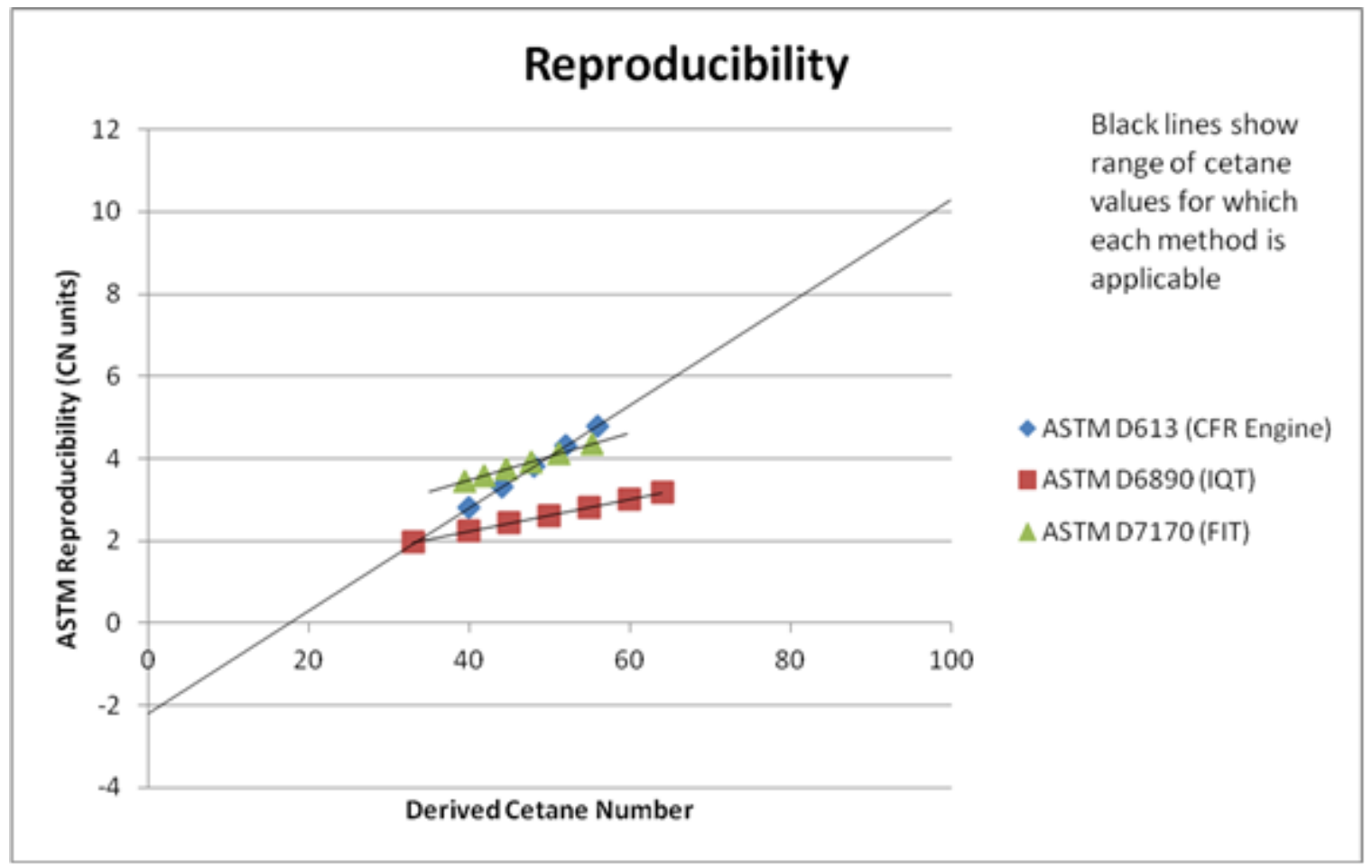

Figure 6. ASTM reproducibility of ASTM D613, ASTM D6890, and ASTM D7170 
These graphs show that within the tested range, the IQT method has the best reproducibility of the three methods and its repeatability is comparable to that of the CFR engine approach. In fact, in this compendium, for the 18 compounds that were measured more than once by IQT, generally in different laboratories with different samples, the standard deviation averaged approximately 3 cetane units, comparable to the reproducibility shown in Figure 6. The FIT method has a lower repeatability than the other two methods, and its reproducibility is comparable to that of the CFR engine approach.

It is well known that the correlation between results from ASTM D613 and D6890 is not perfect for many compounds $[14,16]$ because the methodologies used are not testing exactly the same fuel characteristic. Similarly, it should be expected that the correlation between ASTM D613 and ASTM D7170 (the FIT method) may not correlate exactly for the same reason. Figure 7 shows how measurements made using various approaches compare to the same compound measured in the ASTM D613 CFR engine. Because these tests were done by different laboratories on different samples, some of the variation is likely due to sample impurities. Table 3 shows the average absolute difference in DCN as measured by IQT and other methods.

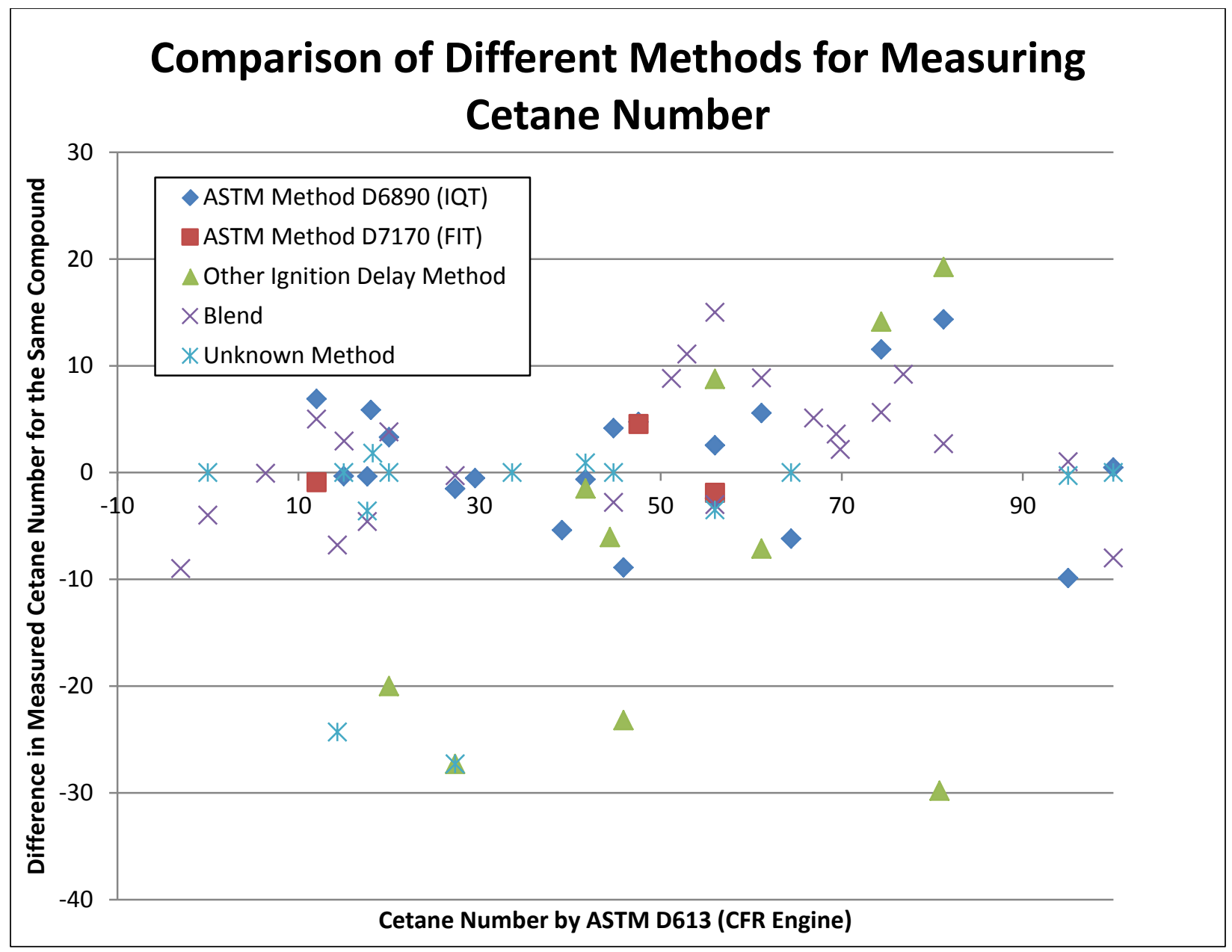

Figure 7. Comparison of different methods for measuring cetane number 
Table 3. Average Absolute Difference between DCN by IQT and Values Measured by Other Methods

\begin{tabular}{llllll}
\hline Method & $\begin{array}{c}\text { ASTM Method } \\
\text { D613 (CFR) }\end{array}$ & $\begin{array}{c}\text { ASTM Method } \\
\text { D7170 (FIT) }\end{array}$ & $\begin{array}{l}\text { Other Ignition } \\
\text { Delay Method }\end{array}$ & Blend & \multicolumn{1}{c}{$\begin{array}{c}\text { Unknown } \\
\text { Method }\end{array}$} \\
\hline Average & $4.8(20$ & $2.5(3$ & $15.7(10$ & $5.4(23$ & $4.4(14$ \\
Difference & compounds $)$ & compounds $)$ & compounds $)$ & compounds $)$ & compounds $)$ \\
\hline
\end{tabular}

Note: The last update to this table was in the 2014 Compendium.

\subsection{Estimation Methods for Cetane Number}

In 1995, Ladommatos [29] and coworkers reviewed numerous methods used to correlate the cetane number with various physical and chemical attributes of a blend or individual compounds in the fuel, but model predictions of cetane number continue to be a fruitful area of research [30, 31, and many more]. These include correlations based on properties such as density, boiling points, and molecular composition. While a thorough review is beyond the scope of this document, Figure 8 shows the correlation between research octane number (RON), as determined using ASTM D2699, and cetane number using all readily available data (cetane number from this compendium and octane numbers from two comprehensive sources [32, 33]). In addition, an earlier correlation, developed by Bowden and coworkers, is included in the graph over the range of values that was considered in their work [27]. In the 2004 compendium, some values of cetane number derived from a correlation with octane number were included in the cetane number tables. In this document, use of the correlation is left to the reader, and only experimentally measured cetane numbers are included in Appendix A.

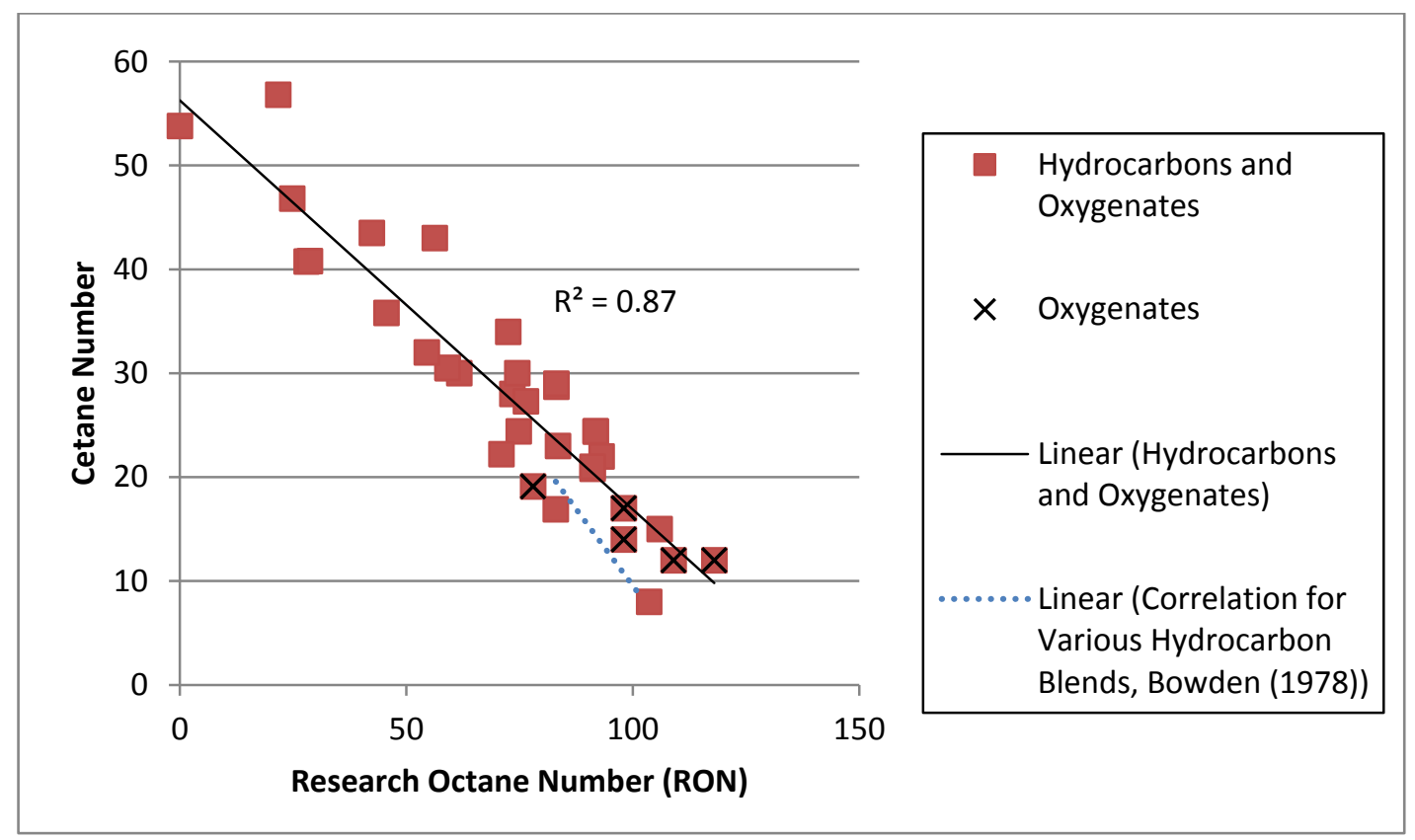

Figure 8. Correlation between cetane number and research octane number suggests cetane number for both oxygenates and hydrocarbons can be roughly estimated as $56-(0.39 \times$ research octane number). 


\section{Summary}

All available experimental cetane number data for individual compounds are included in Appendix A. The listing is organized by compound class and then by molecular formula. The method used to derive the value is noted. Data from empirical cetane number correlations are not included in this compendium, with the exception that early data converted from cetene numbers are included where noted in the Comments column.

Based on this survey, we find:

- A total of 761 measurements of cetane number are reported for a total of 497 compounds.

- In many cases, duplicate data for the same compound do not agree.

- ASTM D6890 and ASTM D7170 are limited by testing that has only investigated accuracy and precision over a very narrow range of DCNs representative of typical diesel fuels.

- Nonetheless, the data collected using ASTM D613, D6890, or D7170 during the past decade represent the most accurate information available. Those data are in the green columns in Appendix A.

- Recent results have demonstrated that the presence of peroxide impurities can make a substantial difference in the measured cetane number.

- The purity of many of these compounds is unknown or is suspect.

- There is no accepted extension of the cetane number scale beyond 0 or 100 despite the need to characterize compounds with lower and higher cetane numbers. 


\section{Appendix A. Compendium of Experimental Cetane Number Data}

A.1 Alkanes

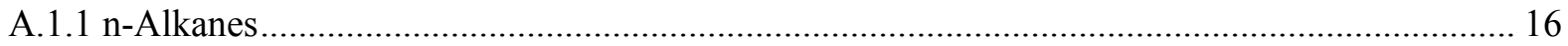

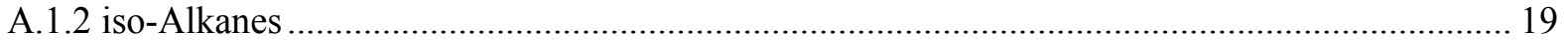

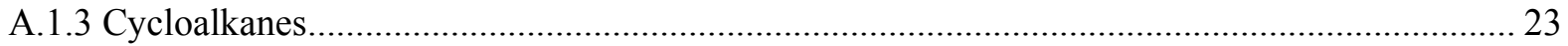

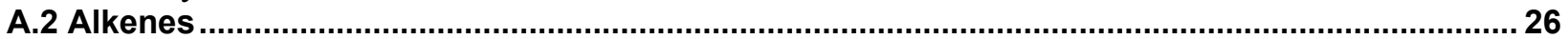

A.3 Aromatics

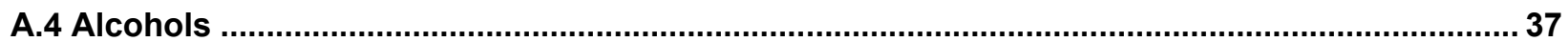

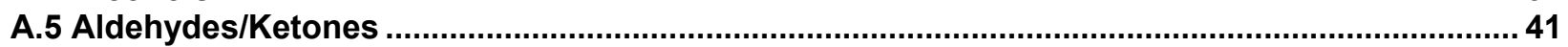

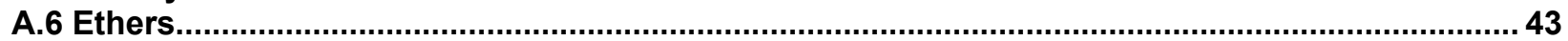

A.7 Esters

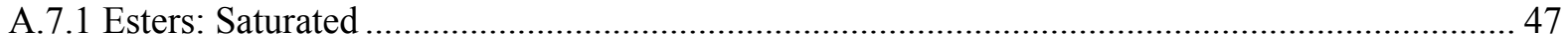

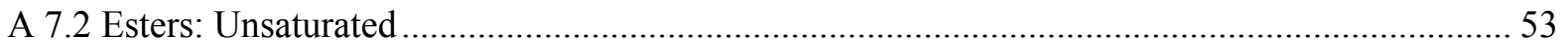

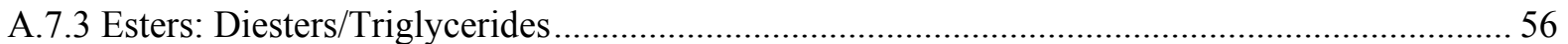

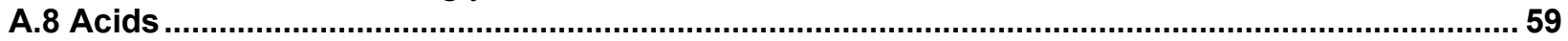

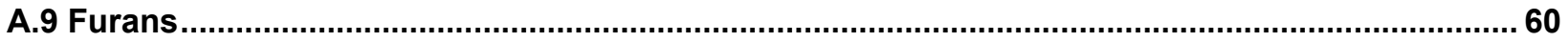

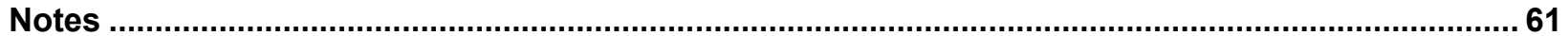




\section{A.1 Alkanes}

\section{A.1.1 n-Alkanes}

\begin{tabular}{|c|c|c|c|c|c|c|c|c|c|c|}
\hline Compound & Formula & CAS & $\begin{array}{c}\text { ASTM } \\
\text { Method } \\
\text { D613 } \\
\text { (CFR) }\end{array}$ & $\begin{array}{c}\text { ASTM } \\
\text { Method } \\
\text { D6890 } \\
\text { (IQT) }\end{array}$ & $\begin{array}{c}\text { ASTM } \\
\text { Method } \\
\text { D7170 } \\
\text { (FIT) }\end{array}$ & $\begin{array}{l}\text { Other } \\
\text { Ignition } \\
\text { Delay } \\
\text { Method }\end{array}$ & Blend & $\begin{array}{l}\text { Unknown } \\
\text { Method }\end{array}$ & Ref. & Notes \\
\hline n-propane & $\mathrm{C} 3 \mathrm{H} 8$ & $74-98-6$ & & & & & & -20 & 37 & \\
\hline n-butane & $\mathrm{C} 4 \mathrm{H} 10$ & $106-97-8$ & & 20.6 & & & & & 77 & new \\
\hline n-pentane & $\mathrm{C} 5 \mathrm{H} 12$ & $109-66-0$ & & & & & 30 & & 1 & [1], [30] \\
\hline \multirow[t]{5}{*}{ n-hexane } & \multirow[t]{5}{*}{$\mathrm{C} 6 \mathrm{H} 14$} & \multirow[t]{5}{*}{$110-54-3$} & 44.8 & & & & & & 9 & \\
\hline & & & & 47.9 & & & & & 41 & [2] \\
\hline & & & & 50.0 & & & & & 49 & \\
\hline & & & & & & & 42 & & 1 & [1], [30] \\
\hline & & & & & & & & 45 & 2 & \\
\hline \multirow[t]{10}{*}{ n-heptane } & \multirow[t]{10}{*}{ C7H16 } & \multirow[t]{10}{*}{$142-82-5$} & 56.0 & & & & & & 3,4 & [27] \\
\hline & & & & 53.8 & & & & & 41 & [2] \\
\hline & & & & 53.0 & & & & & 49 & \\
\hline & & & & 53.7 & & & & & 51 & \\
\hline & & & & 53.8 & & & & & 59 & [4] \\
\hline & & & & & 54.1 & & & & 65 & \\
\hline & & & & & & & 53 & & 1 & [1], [30] \\
\hline & & & & 53.8 & & & & & 72 & new \\
\hline & & & & 53.8 & & & & & 77 & new \\
\hline & & & & 52.8 & & & & & 76 & new \\
\hline
\end{tabular}




\begin{tabular}{|c|c|c|c|c|c|c|c|c|c|c|}
\hline Compound & Formula & CAS & $\begin{array}{l}\text { ASTM } \\
\text { Method } \\
\text { D613 } \\
\text { (CFR) }\end{array}$ & $\begin{array}{l}\text { ASTM } \\
\text { Method } \\
\text { D6890 } \\
\text { (IQT) }\end{array}$ & $\begin{array}{l}\text { ASTM } \\
\text { Method } \\
\text { D7170 } \\
\text { (FIT) }\end{array}$ & $\begin{array}{l}\text { Other } \\
\text { Ignition } \\
\text { Delay } \\
\text { Method }\end{array}$ & Blend & $\begin{array}{l}\text { Unknown } \\
\text { Method }\end{array}$ & Ref. & Notes \\
\hline \multirow[t]{4}{*}{ n-octane } & \multirow[t]{4}{*}{$\mathrm{C} 8 \mathrm{H} 18$} & \multirow[t]{4}{*}{$111-65-9$} & 64.4 & & & & & & 9 & [35] \\
\hline & & & & 58.2 & & & & & 41 & [2] \\
\hline & & & & & & & & 65 & 2 & \\
\hline & & & & & & & & 64 & 5 & [22] \\
\hline \multirow[t]{3}{*}{ n-nonane } & \multirow[t]{3}{*}{$\mathrm{C} 9 \mathrm{H} 2 \mathrm{O}$} & \multirow[t]{3}{*}{$111-84-2$} & & 60.9 & & & & & 41 & \\
\hline & & & & & & & 74 & & 1 & [1], [30] \\
\hline & & & & & & & & 72 & 2,6 & \\
\hline \multirow[t]{7}{*}{ n-decane } & \multirow[t]{7}{*}{$\mathrm{C} 10 \mathrm{H} 22$} & \multirow[t]{7}{*}{$124-18-5$} & & 65.5 & & & & & 41 & \\
\hline & & & & 67.2 & & & & & 64 & \\
\hline & & & & & & & 65 & & 62,63 & [6], [32] \\
\hline & & & & & & & & 78 & 2 & \\
\hline & & & & & & & & 76 & 4 & \\
\hline & & & & & & & & 77 & 5 & [22] \\
\hline & & & & & & & & 76 & 7 & \\
\hline \multirow[t]{2}{*}{ n-undecane } & \multirow[t]{2}{*}{$\mathrm{C} 11 \mathrm{H} 24$} & \multirow[t]{2}{*}{$1120-21-4$} & & & & & 79 & & 1 & [1], [30] \\
\hline & & & & & & & & 83 & 2,6 & \\
\hline \multirow[t]{5}{*}{ n-dodecane } & \multirow[t]{5}{*}{$\mathrm{C} 12 \mathrm{H} 26$} & \multirow[t]{5}{*}{$112-40-3$} & & 72.9 & & & & & 41 & \\
\hline & & & & 74.0 & & & & & 49 & \\
\hline & & & & & & & 78 & & 61,63 & [6],[33] \\
\hline & & & & & & & & 88 & 5 & [22] \\
\hline & & & & & & & & 80 & 3,4 & \\
\hline \multirow[t]{2}{*}{$\mathrm{n}$-tridecane } & \multirow[t]{2}{*}{$\mathrm{C} 13 \mathrm{H} 28$} & \multirow[t]{2}{*}{$629-50-5$} & & & & & & 88 & 4 & \\
\hline & & & & & & & & 91 & 2,6 & \\
\hline
\end{tabular}




\begin{tabular}{|c|c|c|c|c|c|c|c|c|c|c|}
\hline Compound & Formula & CAS & $\begin{array}{l}\text { ASTM } \\
\text { Method } \\
\text { D613 } \\
\text { (CFR) }\end{array}$ & $\begin{array}{l}\text { ASTM } \\
\text { Method } \\
\text { D6890 } \\
\text { (IQT) }\end{array}$ & $\begin{array}{l}\text { ASTM } \\
\text { Method } \\
\text { D7170 } \\
\text { (FIT) }\end{array}$ & $\begin{array}{l}\text { Other } \\
\text { Ignition } \\
\text { Delay } \\
\text { Method }\end{array}$ & Blend & $\begin{array}{l}\text { Unknown } \\
\text { Method }\end{array}$ & Ref. & Notes \\
\hline \multirow[t]{6}{*}{ n-tetradecane } & \multirow[t]{6}{*}{$\mathrm{C} 14 \mathrm{H} 30$} & \multirow[t]{6}{*}{$629-59-4$} & 95.0 & & & & & & 9 & [35] \\
\hline & & & & 85.1 & & & & & 41 & \\
\hline & & & & & & & 96 & & 1 & $\begin{array}{c}\text { [1], [30], } \\
{[35]}\end{array}$ \\
\hline & & & & & & & & 95 & 2 & \\
\hline & & & & & & & & 93 & 4 & \\
\hline & & & & & & & & 96 & 5 & [22] \\
\hline \multirow[t]{2}{*}{ n-pentadecane } & \multirow[t]{2}{*}{$\mathrm{C} 15 \mathrm{H} 32$} & \multirow[t]{2}{*}{$629-62-9$} & & & & & & 98 & 2,6 & \\
\hline & & & & & & & & 95 & 4,7 & \\
\hline \multirow[t]{5}{*}{ n-hexadecane } & \multirow[t]{5}{*}{$\mathrm{C} 16 \mathrm{H} 34$} & \multirow[t]{5}{*}{$544-76-3$} & 100.0 & & & & & & PRF & [9] \\
\hline & & & & 100.5 & & & & & 41 & \\
\hline & & & & & & & 92 & & 1 & [1], [30] \\
\hline & & & & & & & & 100 & 4 & \\
\hline & & & & 98.5 & & & & & 76 & new \\
\hline n-heptadecane & $\mathrm{C} 17 \mathrm{H} 36$ & $629-78-7$ & & & & & & 105 & 4 & \\
\hline \multirow[t]{2}{*}{ n-octadecane } & \multirow[t]{2}{*}{$\mathrm{C} 18 \mathrm{H} 38$} & \multirow[t]{2}{*}{$593-45-3$} & & & & & & 110 & 4 & [5] \\
\hline & & & & & & & & 103 & 5 & {$[5],[22]$} \\
\hline n-nonadecane & $\mathrm{C} 19 \mathrm{H} 40$ & $629-92-5$ & & & & & & 110 & 4 & [5] \\
\hline n-eicosane & $\mathrm{C} 2 \mathrm{OH} 42$ & $12-95-8$ & & & & & & 110 & 4,7 & [5] \\
\hline
\end{tabular}




\section{A.1.2 iso-Alkanes}

\begin{tabular}{|c|c|c|c|c|c|c|c|c|c|c|}
\hline Compound & Formula & CAS & $\begin{array}{l}\text { ASTM } \\
\text { Method } \\
\text { D613 } \\
\text { (CFR) }\end{array}$ & $\begin{array}{c}\text { ASTM } \\
\text { Method } \\
\text { D6890 } \\
\text { (IQT) }\end{array}$ & $\begin{array}{c}\text { ASTM } \\
\text { Method } \\
\text { D7170 } \\
\text { (FIT) }\end{array}$ & $\begin{array}{l}\text { Other } \\
\text { Ignition } \\
\text { Delay } \\
\text { Method }\end{array}$ & Blend & $\begin{array}{l}\text { Unknown } \\
\text { Method }\end{array}$ & Ref. & Notes \\
\hline \multirow[t]{2}{*}{ 2-methylbutane } & \multirow[t]{2}{*}{$\mathrm{C} 5 \mathrm{H} 12$} & \multirow[t]{2}{*}{$78-78-4$} & & 24.0 & & & & & 76 & new \\
\hline & & & & 25.8 & & & & & 77 & new \\
\hline 2,2-dimethylbutane & $\mathrm{C} 6 \mathrm{H} 14$ & $75-83-2$ & & 24.4 & & & & & 41 & \\
\hline \multirow[t]{3}{*}{ 2-methylpentane } & \multirow[t]{3}{*}{$\mathrm{C} 6 \mathrm{H} 14$} & \multirow[t]{3}{*}{$107-83-5$} & & & & & 29 & & 1 & $\begin{array}{c}\text { [1], [23], } \\
{[30]}\end{array}$ \\
\hline & & & & & & & & 33 & 4 & \\
\hline & & & & 34.5 & & & & & 41 & \\
\hline \multirow[t]{2}{*}{ 3-methylpentane } & \multirow[t]{2}{*}{$\mathrm{C} 6 \mathrm{H} 14$} & \multirow[t]{2}{*}{$96-14-0$} & 30.0 & & & & & & 3,4 & [27] \\
\hline & & & & 30.7 & & & & & 77 & new \\
\hline 2,2,3-trimethylbutane & $\mathrm{C} 7 \mathrm{H} 16$ & $464-06-2$ & & 12.9 & & & & & 41 & \\
\hline \multirow[t]{2}{*}{ 2,3-dimethylpentane } & \multirow[t]{2}{*}{$\mathrm{C} 7 \mathrm{H} 16$} & \multirow[t]{2}{*}{$565-59-3$} & & 22.0 & & & & & 41 & \\
\hline & & & & 21.7 & & & & & 77 & new \\
\hline \multirow[t]{3}{*}{ 2,4-dimethylpentane } & \multirow[t]{3}{*}{$\mathrm{C} 7 \mathrm{H} 16$} & \multirow[t]{3}{*}{$108-08-7$} & & & & & 29 & & 1 & $\begin{array}{c}\text { [1], [30], } \\
{[35]}\end{array}$ \\
\hline & & & & 28.7 & & & & & 41 & \\
\hline & & & & 28.2 & & & & & 77 & new \\
\hline 2-methylhexane & $\mathrm{C} 7 \mathrm{H} 16$ & $591-76-4$ & & 43.5 & & & & & 41 & \\
\hline 3-ethylpentane & $\mathrm{C} 7 \mathrm{H} 16$ & $617-78-7$ & & 34.1 & & & & & 41 & \\
\hline 3-methylhexane & $\mathrm{C} 7 \mathrm{H} 16$ & $589-34-4$ & & 42.0 & & & & & 77 & new \\
\hline
\end{tabular}




\begin{tabular}{|c|c|c|c|c|c|c|c|c|c|c|}
\hline Compound & Formula & CAS & $\begin{array}{l}\text { ASTM } \\
\text { Method } \\
\text { D613 } \\
\text { (CFR) }\end{array}$ & $\begin{array}{c}\text { ASTM } \\
\text { Method } \\
\text { D6890 } \\
\text { (IQT) }\end{array}$ & $\begin{array}{c}\text { ASTM } \\
\text { Method } \\
\text { D7170 } \\
\text { (FIT) }\end{array}$ & $\begin{array}{l}\text { Other } \\
\text { Ignition } \\
\text { Delay } \\
\text { Method }\end{array}$ & Blend & $\begin{array}{l}\text { Unknown } \\
\text { Method }\end{array}$ & Ref. & Notes \\
\hline \multirow{9}{*}{$\begin{array}{c}2,2,4- \\
\text { trimethylpentane }\end{array}$} & \multirow[t]{9}{*}{$\mathrm{C} 8 \mathrm{H} 18$} & \multirow[t]{9}{*}{$540-84-1$} & 17.6 & & & & & & 9 & [35] \\
\hline & & & 12.0 & & & & & & 3,4 & [27] \\
\hline & & & & 17.2 & & & & & 41 & \\
\hline & & & & 18.9 & & & & & 58 & \\
\hline & & & & & 11.1 & & & & 65 & \\
\hline & & & & & & & 17 & & $\begin{array}{c}62,6 \\
3\end{array}$ & {$[6],[32]$} \\
\hline & & & & & & & 13 & & 1 & $\begin{array}{c}{[25],[30],} \\
{[37]}\end{array}$ \\
\hline & & & & & & & & 14 & 6 & \\
\hline & & & & 17.4 & & & & & 77 & new \\
\hline \multirow[t]{2}{*}{ 2-methylheptane } & \multirow[t]{2}{*}{$\mathrm{C} 8 \mathrm{H} 18$} & \multirow[t]{2}{*}{$592-27-8$} & & 52.6 & & & & & 41 & \\
\hline & & & & 47.0 & & & & & 54 & \\
\hline \multirow[t]{2}{*}{ 2,2,5-trimethylhexane } & \multirow[t]{2}{*}{$\mathrm{C} 9 \mathrm{H} 20$} & \multirow[t]{2}{*}{$3522-94-9$} & & & & & 24 & & 1 & $\begin{array}{c}\text { [1], [30], } \\
\text { [37] }\end{array}$ \\
\hline & & & & & & & & 24 & 1,8 & \\
\hline 2,2-dimethyloctane & $\mathrm{C} 10 \mathrm{H} 22$ & $15869-87-1$ & & & & & & 59 & 7 & \\
\hline 2,6-dimethyloctane & $\mathrm{C} 10 \mathrm{H} 22$ & 2051-30-1 & & 51.7 & & & & & 41 & [7] \\
\hline \multirow{2}{*}{$\begin{array}{c}2,2,4,6,6- \\
\text { pentamethylheptane }\end{array}$} & \multirow[t]{2}{*}{$\mathrm{C} 12 \mathrm{H} 26$} & \multirow[t]{2}{*}{$13475-82-6$} & & & & & & 9 & 3 & [8] \\
\hline & & & & & & & & 9 & 4,11 & \\
\hline \multirow[t]{2}{*}{ 3-ethyldecane } & \multirow[t]{2}{*}{$\mathrm{C} 12 \mathrm{H} 26$} & & & & & & & 48 & 3 & [8], [23] \\
\hline & & & & & & & & 48 & 7,10 & \\
\hline \multirow[t]{2}{*}{ 4,5-diethyloctane } & \multirow[t]{2}{*}{$\mathrm{C} 12 \mathrm{H} 26$} & \multirow[t]{2}{*}{$1636-41-5$} & & & & & & 20 & 3 & [8] \\
\hline & & & & & & & & 20 & 7 & \\
\hline 2,5-dimethylundecane & $\mathrm{C} 13 \mathrm{H} 28$ & $17301-22-3$ & & & & & & 58 & 3 & [8] \\
\hline
\end{tabular}




\begin{tabular}{|c|c|c|c|c|c|c|c|c|c|c|}
\hline Compound & Formula & CAS & $\begin{array}{c}\text { ASTM } \\
\text { Method } \\
\text { D613 } \\
\text { (CFR) }\end{array}$ & $\begin{array}{c}\text { ASTM } \\
\text { Method } \\
\text { D6890 } \\
\text { (IQT) }\end{array}$ & $\begin{array}{c}\text { ASTM } \\
\text { Method } \\
\text { D7170 } \\
\text { (FIT) }\end{array}$ & $\begin{array}{l}\text { Other } \\
\text { Ignition } \\
\text { Delay } \\
\text { Method }\end{array}$ & Blend & $\begin{array}{l}\text { Unknown } \\
\text { Method }\end{array}$ & Ref. & Notes \\
\hline 4-propyldecane & $\mathrm{C} 13 \mathrm{H} 28$ & & & & & & & 39 & 3,4 & [8] \\
\hline 5-butylnonane & $\mathrm{C} 13 \mathrm{H} 28$ & $17312-63-9$ & & & & & & 53 & 3,4 & [8] \\
\hline $\begin{array}{l}\text { 2,7-dimethyl-4,5- } \\
\text { diethyloctane }\end{array}$ & $\mathrm{C} 14 \mathrm{H} 30$ & & & & & & & 39 & 3,4 & [8] \\
\hline \multirow{2}{*}{$\begin{array}{l}\text { farnesane }(2,6,10- \\
\text { trimethyldodecane })\end{array}$} & \multirow[t]{2}{*}{$\mathrm{C} 15 \mathrm{H} 32$} & \multirow[t]{2}{*}{$3891-98-3$} & & 58.0 & & & & & 50 & \\
\hline & & & & 59.1 & & & & & 60 & \\
\hline \multirow{5}{*}{$\begin{array}{c}2,2,4,4,6,8,8- \\
\text { heptamethylnonane }\end{array}$} & \multirow[t]{5}{*}{$\mathrm{C} 16 \mathrm{H} 34$} & \multirow[t]{5}{*}{ 4390-04-9 } & 15.0 & & & & & & PRF & [9] \\
\hline & & & & 15.1 & & & & & 41 & \\
\hline & & & & 14.2 & & & & & 64 & \\
\hline & & & & & & & & 15 & 7 & \\
\hline & & & & 14.2 & & & & & 76 & new \\
\hline 5-butyldodecane & $\mathrm{C} 16 \mathrm{H} 34$ & $6118-01-0$ & & & & & & 45 & 4 & \\
\hline $\begin{array}{c}7,8- \\
\text { dimethyltetradecane }\end{array}$ & $\mathrm{C} 16 \mathrm{H} 34$ & $2801-86-7$ & & & & & & 40 & 3,4 & [8] \\
\hline 7-butyltridecane & $\mathrm{C} 17 \mathrm{H} 36$ & & & & & & & 70 & 3,4 & [8] \\
\hline 2-methylheptadecane & $\mathrm{C} 18 \mathrm{H} 38$ & $18869-72-2$ & & 91.0 & & & & & 41 & \\
\hline 5,6-dibutyldecane & $\mathrm{C} 18 \mathrm{H} 38$ & & & & & & & 30 & 3,4 & [8] \\
\hline \multirow[t]{2}{*}{ 7,8-diethyltetradecane } & \multirow[t]{2}{*}{$\mathrm{C} 18 \mathrm{H} 38$} & \multirow[t]{2}{*}{$500020-70-2$} & & & & & & 67 & 3 & [8] \\
\hline & & & & & & & & 67 & 4,11 & \\
\hline \multirow[t]{2}{*}{ 8-propylpentadecane } & \multirow[t]{2}{*}{$\mathrm{C} 18 \mathrm{H} 38$} & & & & & & & 48 & 3 & [8] \\
\hline & & & & & & & & 48 & 4,7 & \\
\hline 9-methylheptadecane & C18H38 & $18869-72-2$ & & & & & & 66 & 3,4 & [8] \\
\hline 2-methyloctadecane & $\mathrm{C} 19 \mathrm{H} 40$ & $1560-88-9$ & & 104.4 & & & & & 41 & \\
\hline
\end{tabular}




\begin{tabular}{|c|c|c|c|c|c|c|c|c|c|c|}
\hline Compound & Formula & CAS & $\begin{array}{l}\text { ASTM } \\
\text { Method } \\
\text { D613 } \\
\text { (CFR) }\end{array}$ & $\begin{array}{c}\text { ASTM } \\
\text { Method } \\
\text { D6890 } \\
\text { (IQT) }\end{array}$ & $\begin{array}{l}\text { ASTM } \\
\text { Method } \\
\text { D7170 } \\
\text { (FIT) }\end{array}$ & $\begin{array}{l}\text { Other } \\
\text { Ignition } \\
\text { Delay } \\
\text { Method }\end{array}$ & Blend & $\begin{array}{l}\text { Unknown } \\
\text { Method }\end{array}$ & Ref. & Notes \\
\hline $\begin{array}{c}9,10- \\
\text { dimethyloctadecane }\end{array}$ & $\mathrm{C} 2 \mathrm{OH} 42$ & & & & & & & 60 & 3,4 & [8] \\
\hline 7-hexylpentadecane & $\mathrm{C} 21 \mathrm{H} 44$ & & & & & & & 83 & 3,4 & [8] \\
\hline $\begin{array}{l}\text { 2,9-dimethyl-5,6- } \\
\text { diisopentyldecane }\end{array}$ & $\mathrm{C} 22 \mathrm{H} 46$ & & & & & & & 48 & 3,4 & [8] \\
\hline $\begin{array}{c}10,13- \\
\text { dimethyldocosane }\end{array}$ & $\mathrm{C} 24 \mathrm{H} 50$ & & & & & & & 56 & 11 & \\
\hline $\begin{array}{c}9,10- \\
\text { dipropyloctadecane }\end{array}$ & $\mathrm{C} 24 \mathrm{H} 50$ & & & & & & & 47 & 3,4 & [8] \\
\hline 9-heptylheptadecane & $\mathrm{C} 24 \mathrm{H} 50$ & & & & & & & 88 & 3,4 & [8] \\
\hline
\end{tabular}




\section{A.1.3 Cycloalkanes}

\begin{tabular}{|c|c|c|c|c|c|c|c|c|c|c|}
\hline Compound & Formula & CAS & $\begin{array}{l}\text { ASTM } \\
\text { Method } \\
\text { D613 } \\
\text { (CFR) }\end{array}$ & $\begin{array}{c}\text { ASTM } \\
\text { Method } \\
\text { D6890 } \\
\text { (IQT) }\end{array}$ & $\begin{array}{c}\text { ASTM } \\
\text { Method } \\
\text { D7170 } \\
\text { (FIT) }\end{array}$ & $\begin{array}{l}\text { Other } \\
\text { Ignition } \\
\text { Delay } \\
\text { Method }\end{array}$ & Blend & $\begin{array}{l}\text { Unkno } \\
\text { wn } \\
\text { Method }\end{array}$ & Ref. & Notes \\
\hline cyclopentane & $\mathrm{C} 5 \mathrm{H} 10$ & $287-92-3$ & & 6.1 & & & & & 41 & \\
\hline \multirow[t]{5}{*}{ cyclohexane } & \multirow[t]{5}{*}{$\mathrm{C} 6 \mathrm{H} 12$} & \multirow[t]{5}{*}{$110-82-7$} & 16.9 & & & & & & 9 & [35] \\
\hline & & & 13.2 & & & & & & $3,4,6$ & [27] \\
\hline & & & & & & & 18 & & 1 & [1], [30] \\
\hline & & & & 19.9 & & & & & 77 & new \\
\hline & & & & 20.0 & & & & & 76 & new \\
\hline methylcyclopentane & $\mathrm{C} 6 \mathrm{H} 12$ & $96-37-7$ & & 17.2 & & & & & 41 & \\
\hline \multirow[t]{6}{*}{ methylcyclohexane } & \multirow[t]{6}{*}{$\mathrm{C} 7 \mathrm{H} 14$} & \multirow[t]{6}{*}{$108-87-2$} & 20.0 & & & & & & 3,4 & {$[27]$} \\
\hline & & & & 24.4 & & & & & 41 & [7] \\
\hline & & & & 22.0 & & & & & 55 & \\
\hline & & & & 23.5 & & & & & 59 & {$[4]$} \\
\hline & & & & & & & 24 & & 1 & $\begin{array}{c}{[1],[30],} \\
{[37]}\end{array}$ \\
\hline & & & & & & & & 20 & 5 & [22] \\
\hline $\begin{array}{c}1,2- \\
\text { dimethylcyclohexane }\end{array}$ & $\mathrm{C} 8 \mathrm{H} 16$ & $583-57-3$ & & 24.0 & & & & & 77 & new \\
\hline $\begin{array}{c}1,3- \\
\text { dimethylcyclohexane }\end{array}$ & $\mathrm{C} 8 \mathrm{H} 16$ & $591-21-9$ & & 30.5 & & & & & 77 & new \\
\hline cyclooctane & $\mathrm{C} 8 \mathrm{H} 16$ & $292-64-8$ & & 22.3 & & & & & 41 & \\
\hline ethylcyclohexane & $\mathrm{C} 8 \mathrm{H} 16$ & $1678-91-7$ & & 35.8 & & & & & 41 & \\
\hline $\begin{array}{c}1,2,4- \\
\text { trimethylcyclohexane }\end{array}$ & $\mathrm{C} 9 \mathrm{H} 18$ & $2234-75-5$ & & 24.4 & & & & & 76 & new \\
\hline $\begin{array}{c}1,3,5- \\
\text { trimethylcyclohexane }\end{array}$ & $\mathrm{C9H} 18$ & $1839-63-0$ & & 30.5 & & & & & $\begin{array}{l}41 \\
54\end{array}$ & \\
\hline
\end{tabular}




\begin{tabular}{|c|c|c|c|c|c|c|c|c|c|c|}
\hline Compound & Formula & CAS & $\begin{array}{l}\text { ASTM } \\
\text { Method } \\
\text { D613 } \\
\text { (CFR) }\end{array}$ & $\begin{array}{c}\text { ASTM } \\
\text { Method } \\
\text { D6890 } \\
\text { (IQT) }\end{array}$ & $\begin{array}{c}\text { ASTM } \\
\text { Method } \\
\text { D7170 } \\
\text { (FIT) }\end{array}$ & $\begin{array}{l}\text { Other } \\
\text { Ignition } \\
\text { Delay } \\
\text { Method }\end{array}$ & Blend & $\begin{array}{c}\text { Unkno } \\
\text { wn } \\
\text { Method }\end{array}$ & Ref. & Notes \\
\hline $\begin{array}{c}\text { tetrahydro- } \\
\text { dicyclopentadiene }\end{array}$ & $\mathrm{C} 10 \mathrm{H} 16$ & $2825-82-3$ & & 20.4 & & & & & 74 & new \\
\hline \multirow[t]{2}{*}{ cis-decalin } & \multirow[t]{2}{*}{$\mathrm{C} 10 \mathrm{H} 18$} & \multirow[t]{2}{*}{$91-17-8$} & & 39.4 & & & & & 41 & [2] \\
\hline & & & & 41.6 & & & & & 55 & \\
\hline \multirow[t]{3}{*}{ decalin } & \multirow[t]{3}{*}{$\mathrm{C} 10 \mathrm{H} 18$} & \multirow[t]{3}{*}{$91-17-8$} & & & & & & 48 & 3 & {$[8],[36]$} \\
\hline & & & & & & & & 42 & 5 & [22] \\
\hline & & & & & & & & 48 & 4,7 & {$[36]$} \\
\hline \multirow[t]{2}{*}{ trans-decalin } & \multirow[t]{2}{*}{$\mathrm{C} 10 \mathrm{H} 18$} & \multirow[t]{2}{*}{$91-17-8$} & & 31.8 & & & & & 41 & \\
\hline & & & & 32.0 & & & & & 55 & \\
\hline \multirow[t]{2}{*}{ n-butylcyclohexane } & \multirow[t]{2}{*}{$\mathrm{C} 10 \mathrm{H} 20$} & \multirow[t]{2}{*}{$1678-93-9$} & & 47.6 & & & & & 41 & \\
\hline & & & & 48.0 & & & & & 54 & $\begin{array}{c}\text { [3] old } \\
\text { value } \\
\text { was } 46.5\end{array}$ \\
\hline 2-ethyladamantane & $\mathrm{C} 12 \mathrm{H} 20$ & & & 42.7 & & & & & 75 & new \\
\hline \multirow[t]{2}{*}{ bicyclohexyl } & \multirow[t]{2}{*}{$\mathrm{C} 12 \mathrm{H} 22$} & \multirow[t]{2}{*}{$92-51-3$} & & & & & & 47 & 5 & [22] \\
\hline & & & & & & & & 53 & 3,4 & \\
\hline \multirow[t]{2}{*}{ 3-cyclohexylhexane } & \multirow[t]{2}{*}{$\mathrm{C} 12 \mathrm{H} 24$} & & & & & & & 36 & 3 & [8] \\
\hline & & & & & & & & 36 & 4,7 & \\
\hline propyladamantane & $\mathrm{C} 13 \mathrm{H} 22$ & & & 48.6 & & & & & 75 & new \\
\hline n-propyldecalin & $\mathrm{C} 13 \mathrm{H} 24$ & & & & & & & 35 & 3,4 & [8] \\
\hline butyladamantane & $\mathrm{C} 14 \mathrm{H} 24$ & $14449-41-3$ & & 49.1 & & & & & 75 & new \\
\hline perhydrophenanthrene & $\mathrm{C} 14 \mathrm{H} 24$ & $5743-97-5$ & & 38.8 & & & & & 41 & [7] \\
\hline n-butyldecalin & $\mathrm{C} 14 \mathrm{H} 26$ & & & & & & & 31 & 3,4 & [8] \\
\hline sec-butyldecalin & $\mathrm{C} 14 \mathrm{H} 26$ & & & & & & & 34 & 3,4 & [8] \\
\hline tert-butyldecalin & $\mathrm{C} 14 \mathrm{H} 26$ & & & & & & & 24 & 3,4 & [8] \\
\hline
\end{tabular}




\begin{tabular}{|c|c|c|c|c|c|c|c|c|c|c|}
\hline Compound & Formula & CAS & $\begin{array}{l}\text { ASTM } \\
\text { Method } \\
\text { D613 } \\
\text { (CFR) }\end{array}$ & $\begin{array}{l}\text { ASTM } \\
\text { Method } \\
\text { D6890 } \\
\text { (IQT) }\end{array}$ & $\begin{array}{c}\text { ASTM } \\
\text { Method } \\
\text { D7170 } \\
\text { (FIT) }\end{array}$ & $\begin{array}{l}\text { Other } \\
\text { Ignition } \\
\text { Delay } \\
\text { Method }\end{array}$ & Blend & $\begin{array}{l}\text { Unkno } \\
\text { wn } \\
\text { Method }\end{array}$ & Ref. & Notes \\
\hline pentyladamantane & $\mathrm{C} 15 \mathrm{H} 26$ & & & 48.5 & & & & & 75 & new \\
\hline $\begin{array}{c}1,3,5- \\
\text { triisopropylcyclohexane }\end{array}$ & $\mathrm{C} 15 \mathrm{H} 30$ & $34387-60-5$ & & 25.3 & & & & & 41 & \\
\hline \multirow{2}{*}{$\begin{array}{l}\text { 2-methyl-3- } \\
\text { cyclohexylnonane }\end{array}$} & \multirow[t]{2}{*}{$\mathrm{C} 16 \mathrm{H} 32$} & & & & & & & 56 & 3 & [8] \\
\hline & & & & & & & & 70 & 7 & \\
\hline n-octyldecalin & $\mathrm{C} 18 \mathrm{H} 34$ & & & & & & & 31 & 3,4 & [8] \\
\hline $\begin{array}{c}\text { 1-methyl-3- } \\
\text { dodecylcyclohexane }\end{array}$ & $\mathrm{C} 19 \mathrm{H} 38$ & & & & & & & 70 & 3,4 & [8] \\
\hline \multirow[t]{2}{*}{ 2-cyclohexyltetradecane } & \multirow[t]{2}{*}{$\mathrm{C} 2 \mathrm{OH} 40$} & & & & & & & 57 & 7 & \\
\hline & & & & & & & & 57 & 3,4 & [8] \\
\hline $\begin{array}{l}\text { 2-methyl-2- } \\
\text { cyclohexylpentadecane }\end{array}$ & $\mathrm{C} 22 \mathrm{H} 44$ & & & & & & & 45 & 3,4 & [8] \\
\hline $\begin{array}{c}\text { 1,2,4-trimethyl-5- } \\
\text { hexadecylcyclohexane }\end{array}$ & $\mathrm{C} 25 \mathrm{H} 50$ & & & & & & & 42 & 3,4 & [8] \\
\hline 5-cyclohexyleicosane & $\mathrm{C} 26 \mathrm{H} 52$ & & & & & & & 66 & 3,4 & [8] \\
\hline
\end{tabular}




\section{A.2 Alkenes}

\begin{tabular}{|c|c|c|c|c|c|c|c|c|c|c|}
\hline Compound & Formula & CAS & $\begin{array}{l}\text { ASTM } \\
\text { Method } \\
\text { D613 } \\
\text { (CFR) }\end{array}$ & $\begin{array}{l}\text { ASTM } \\
\text { Method } \\
\text { D6890 } \\
\text { (IQT) }\end{array}$ & $\begin{array}{l}\text { ASTM } \\
\text { Method } \\
\text { D7170 } \\
\text { (FIT) }\end{array}$ & $\begin{array}{l}\text { Other } \\
\text { Ignition } \\
\text { Delay } \\
\text { Method }\end{array}$ & Blend & $\begin{array}{l}\text { Unknown } \\
\text { Method }\end{array}$ & Ref. & Notes \\
\hline 2-methyl-1,3-butadiene & $\mathrm{C} 5 \mathrm{H} 8$ & $78-79-5$ & & 13.0 & & & & & 76 & new \\
\hline \multirow[t]{2}{*}{ Cyclohexene } & \multirow[t]{2}{*}{$\mathrm{C} 6 \mathrm{H} 10$} & \multirow[t]{2}{*}{$110-83-8$} & & & & & 57 & & 1 & $\begin{array}{c}\text { [1], [30], } \\
{[37]}\end{array}$ \\
\hline & & & & 18.1 & & & & & 76 & new \\
\hline \multirow[t]{4}{*}{ 1-hexene } & \multirow[t]{4}{*}{$\mathrm{C} 6 \mathrm{H} 12$} & \multirow[t]{4}{*}{$592-41-6$} & 27.3 & & & & & & 9 & [35] \\
\hline & & & & 25.8 & & & & & 41 & \\
\hline & & & & & & & 27 & & 1 & [1], [30] \\
\hline & & & & 28.9 & & & & & 77 & new \\
\hline 1-heptyne & $\mathrm{C} 7 \mathrm{H} 12$ & $628-71-7$ & & 22.0 & & & & & 41 & \\
\hline 4-methyl-1-cyclohexene & $\mathrm{C} 7 \mathrm{H} 12$ & $591-47-9$ & & & & & 52 & & 1 & [1], [30] \\
\hline 1-heptene & $\mathrm{C} 7 \mathrm{H} 14$ & $592-76-7$ & & 32.0 & & & & & 41 & \\
\hline cis-2-heptene & $\mathrm{C} 7 \mathrm{H} 14$ & $14686-13-6$ & & & & & 44 & & 1 & $\begin{array}{c}{[1],[10],} \\
{[30]}\end{array}$ \\
\hline 1,5-cyclooctadiene & $\mathrm{C} 8 \mathrm{H} 12$ & $111-78-4$ & & 25.7 & & & & & 76 & new \\
\hline 4-vinyl-1-cyclohexene & $\mathrm{C} 8 \mathrm{H} 12$ & $100-40-3$ & & & & & 40 & & 1 & [1], [30] \\
\hline $\begin{array}{l}\text { 2,5-dimethyl-2,4- } \\
\text { hexadiene }\end{array}$ & $\mathrm{C} 8 \mathrm{H} 14$ & $764-13-6$ & & 19.9 & & & & & 76 & new \\
\hline
\end{tabular}




\begin{tabular}{|c|c|c|c|c|c|c|c|c|c|c|}
\hline Compound & Formula & CAS & $\begin{array}{c}\text { ASTM } \\
\text { Method } \\
\text { D613 } \\
\text { (CFR) }\end{array}$ & $\begin{array}{c}\text { ASTM } \\
\text { Method } \\
\text { D6890 } \\
\text { (IQT) }\end{array}$ & $\begin{array}{c}\text { ASTM } \\
\text { Method } \\
\text { D7170 } \\
\text { (FIT) } \\
\end{array}$ & $\begin{array}{c}\text { Other } \\
\text { Ignition } \\
\text { Delay } \\
\text { Method }\end{array}$ & Blend & $\begin{array}{l}\text { Unknown } \\
\text { Method }\end{array}$ & Ref. & Notes \\
\hline \multirow[t]{3}{*}{ 1-octene } & \multirow[t]{3}{*}{$\mathrm{C} 8 \mathrm{H} 16$} & \multirow[t]{3}{*}{$111-66-0$} & & 40.0 & & & & & 41 & \\
\hline & & & & & & & & 41 & 2,6 & \\
\hline & & & & & & & & 41 & 5 & [22] \\
\hline \multirow{4}{*}{$\begin{array}{l}\text { 2,4,4-trimethyl-1- } \\
\text { pentene }\end{array}$} & \multirow[t]{4}{*}{$\mathrm{C} 8 \mathrm{H} 16$} & \multirow[t]{4}{*}{$107-39-1$} & & & & & 11 & & 1 & [1], [30] \\
\hline & & & & & & & & 10 & 4 & \\
\hline & & & & 8.8 & & & & & 77 & new \\
\hline & & & & & & & -3 & & 1 & $\begin{array}{c}{[1],[30],} \\
{[37]}\end{array}$ \\
\hline 2-octene & $\mathrm{C} 8 \mathrm{H} 16$ & $111-67-1$ & & & & & 43 & & 1 & [1], [30] \\
\hline cis-3-octene & $\mathrm{C} 8 \mathrm{H} 16$ & $14850-22-7$ & & 38.1 & & & & & 48 & \\
\hline trans-3-octene & $\mathrm{C} 8 \mathrm{H} 16$ & $14919-01-8$ & & 34.0 & & & & & 48 & \\
\hline 1-nonene & $\mathrm{C} 9 \mathrm{H} 18$ & $124-11-8$ & & & & & & 51 & 2,6 & \\
\hline 2,4-dimethyl-2-heptene & $\mathrm{C} 9 \mathrm{H} 18$ & $860116-58-1$ & & 27.0 & & & & & 73 & new \\
\hline 2,6-dimethylheptene & $\mathrm{C} 9 \mathrm{H} 20$ & $1072-05-5$ & & & & & 51 & & 1 & [1], [30] \\
\hline (R )-(+ )-limonene & $\mathrm{C} 10 \mathrm{H} 16$ & $5989-27-5$ & & 19.0 & & & & & 73 & new \\
\hline 3-carene & $\mathrm{C} 10 \mathrm{H} 16$ & $13466-78-9$ & & 27.0 & & & & & 41 & new \\
\hline limonene & $\mathrm{C} 10 \mathrm{H} 16$ & $138-86-3$ & & 18.9 & & & & & 76 & new \\
\hline
\end{tabular}




\begin{tabular}{|c|c|c|c|c|c|c|c|c|c|c|}
\hline Compound & Formula & CAS & $\begin{array}{l}\text { ASTM } \\
\text { Method } \\
\text { D613 } \\
\text { (CFR) }\end{array}$ & $\begin{array}{c}\text { ASTM } \\
\text { Method } \\
\text { D6890 } \\
\text { (IQT) }\end{array}$ & $\begin{array}{l}\text { ASTM } \\
\text { Method } \\
\text { D7170 } \\
\text { (FIT) }\end{array}$ & $\begin{array}{l}\text { Other } \\
\text { Ignition } \\
\text { Delay } \\
\text { Method }\end{array}$ & Blend & $\begin{array}{l}\text { Unknown } \\
\text { Method }\end{array}$ & Ref. & Notes \\
\hline ocimene & $\mathrm{C} 10 \mathrm{H} 16$ & $13877-91-3$ & & 28.0 & & & & & 41 & new \\
\hline \multirow[t]{3}{*}{ a-pinene } & \multirow[t]{3}{*}{$\mathrm{C} 10 \mathrm{H} 16$} & \multirow[t]{3}{*}{$80-56-8$} & & 18.9 & & & & & 76 & new \\
\hline & & & & 23.5 & & & & & 41 & [7] \\
\hline & & & & 27.0 & & & & & 73 & new \\
\hline \multirow[t]{2}{*}{$\beta$-pinene } & \multirow[t]{2}{*}{$\mathrm{C} 10 \mathrm{H} 16$} & \multirow[t]{2}{*}{$127-91-3$} & & 20.5 & & & & & 76 & new \\
\hline & & & & 22.0 & & & & & 41 & [7] \\
\hline $\mathrm{y}$-terpinene & $\mathrm{C} 10 \mathrm{H} 16$ & $99-85-4$ & & 20.3 & & & & & 76 & new \\
\hline 1,9-decadiene & $\mathrm{C} 10 \mathrm{H} 18$ & $1647-16-1$ & & 41.0 & & & & & 41 & [2] \\
\hline \multirow[t]{4}{*}{ 1-decene } & \multirow[t]{4}{*}{$\mathrm{C} 10 \mathrm{H} 20$} & \multirow[t]{4}{*}{$872-05-9$} & & 49.1 & & & & & 41 & \\
\hline & & & & & & & & 60 & 2 & \\
\hline & & & & & & & & 60 & 5 & {$[22],[23]$} \\
\hline & & & & & & & & 59 & 2,6 & \\
\hline \multirow[t]{2}{*}{ 1-undecene } & \multirow[t]{2}{*}{$\mathrm{C} 11 \mathrm{H} 22$} & \multirow[t]{2}{*}{$821-95-4$} & & & & & & 66 & 2 & \\
\hline & & & & & & & & 65 & 2,6 & \\
\hline
\end{tabular}




\begin{tabular}{|c|c|c|c|c|c|c|c|c|c|c|}
\hline Compound & Formula & CAS & $\begin{array}{l}\text { ASTM } \\
\text { Method } \\
\text { D613 } \\
\text { (CFR) }\end{array}$ & $\begin{array}{l}\text { ASTM } \\
\text { Method } \\
\text { D6890 } \\
\text { (IQT) }\end{array}$ & $\begin{array}{l}\text { ASTM } \\
\text { Method } \\
\text { D7170 } \\
\text { (FIT) }\end{array}$ & $\begin{array}{l}\text { Other } \\
\text { Ignition } \\
\text { Delay } \\
\text { Method }\end{array}$ & Blend & $\begin{array}{l}\text { Unknown } \\
\text { Method }\end{array}$ & Ref. & Notes \\
\hline \multirow[t]{3}{*}{ 1-dodecene } & \multirow[t]{3}{*}{$\mathrm{C} 12 \mathrm{H} 24$} & \multirow[t]{3}{*}{$112-41-4$} & & 56.8 & & & & & 41 & \\
\hline & & & & & & & & 71 & 2,6 & \\
\hline & & & & & & & & 71 & 5 & [22] \\
\hline \multirow[t]{3}{*}{ 1-tetradecene } & \multirow[t]{3}{*}{$\mathrm{C} 14 \mathrm{H} 28$} & \multirow[t]{3}{*}{$1120-36-1$} & & & & & & 83 & 5 & {$[22]$} \\
\hline & & & & & & & & 81 & 2,6 & \\
\hline & & & & & & & & 79 & 3,4 & [8] \\
\hline bisabolene & $\mathrm{C} 15 \mathrm{H} 24$ & & & 32.2 & & & & & 41 & [7] \\
\hline caryophyllene & $\mathrm{C} 15 \mathrm{H} 24$ & $87-44-5$ & & 29.0 & & & & & 41 & new \\
\hline farnesene & $\mathrm{C} 15 \mathrm{H} 24$ & $502-61-4$ & & 32.0 & & & & & 41 & new \\
\hline $\begin{array}{c}\text { 2,6,7-trimethyl-2,6- } \\
\text { tridecadiene }\end{array}$ & $\mathrm{C} 16 \mathrm{H} 30$ & & & & & & & 24 & 3,4 & [8] \\
\hline \multirow[t]{4}{*}{ 1-hexadecene } & \multirow[t]{4}{*}{$\mathrm{C} 16 \mathrm{H} 32$} & \multirow[t]{4}{*}{$629-73-2$} & & 75.9 & & & & & 41 & \\
\hline & & & & & & & & 86 & 2,6 & \\
\hline & & & & & & & & 88 & 3 & \\
\hline & & & & & & & & 84 & 5 & [22] \\
\hline $\begin{array}{c}\text { 2,2,6,6,8,8-hexamethyl- } \\
\text { 4-methylene-nonane }\end{array}$ & $\mathrm{C} 16 \mathrm{H} 32$ & $15220-85-6$ & & & & & & 5 & 3 & \\
\hline 4-butyl-4-dodecene & $\mathrm{C} 16 \mathrm{H} 32$ & & & & & & & 45 & 11 & \\
\hline
\end{tabular}




\begin{tabular}{|c|c|c|c|c|c|c|c|c|c|c|}
\hline Compound & Formula & CAS & $\begin{array}{l}\text { ASTM } \\
\text { Method } \\
\text { D613 } \\
\text { (CFR) }\end{array}$ & $\begin{array}{l}\text { ASTM } \\
\text { Method } \\
\text { D6890 } \\
\text { (IQT) }\end{array}$ & $\begin{array}{c}\text { ASTM } \\
\text { Method } \\
\text { D7170 } \\
\text { (FIT) }\end{array}$ & $\begin{array}{l}\text { Other } \\
\text { Ignition } \\
\text { Delay } \\
\text { Method }\end{array}$ & Blend & $\begin{array}{l}\text { Unknown } \\
\text { Method }\end{array}$ & Ref. & Notes \\
\hline 5-butyl-4-dodecene & $\mathrm{C} 16 \mathrm{H} 32$ & & & & & & & 45 & 3,4 & [8] \\
\hline 7-butyltridecene & $\mathrm{C} 17 \mathrm{H} 34$ & & & & & & & 36 & 3,4 & [8] \\
\hline $\begin{array}{c}\text { 3,12-diethyl-3,11- } \\
\text { tetradecadiene }\end{array}$ & $\mathrm{C} 18 \mathrm{H} 34$ & & & & & & & 26 & 3,4 & [8] \\
\hline 1-octadecene & $\mathrm{C} 18 \mathrm{H} 36$ & $112-88-9$ & & & & & & 90 & 5 & [22] \\
\hline $\begin{array}{l}\text { 7,10-dimethyl-8- } \\
\text { hexadecene }\end{array}$ & $\mathrm{C} 18 \mathrm{H} 36$ & & & & & & & 43 & 3,4 & [8] \\
\hline 8-propyl-8-pentadecene & $\mathrm{C} 18 \mathrm{H} 36$ & & & & & & & 45 & 3,4 & [8] \\
\hline $\begin{array}{l}\text { 9-methyl-9- } \\
\text { heptadecene }\end{array}$ & $\mathrm{C} 18 \mathrm{H} 36$ & & & & & & & 66 & 3,4 & [8] \\
\hline 7-hexyl-7-pentadecene & $\mathrm{C} 21 \mathrm{H} 42$ & & & & & & & 47 & 3,4 & [8] \\
\hline $\begin{array}{c}\text { 10,13-dimethyl-11- } \\
\text { doeicosene }\end{array}$ & $\mathrm{C} 24 \mathrm{H} 48$ & & & & & & & 56 & 3,4 & [8] \\
\hline
\end{tabular}




\section{A.3 Aromatics}

\begin{tabular}{|c|c|c|c|c|c|c|c|c|c|c|}
\hline Compound & Formula & CAS & $\begin{array}{l}\text { ASTM } \\
\text { Method } \\
\text { D613 } \\
\text { (CFR) }\end{array}$ & $\begin{array}{l}\text { ASTM } \\
\text { Method } \\
\text { D6890 } \\
\text { (IQT) }\end{array}$ & $\begin{array}{l}\text { ASTM } \\
\text { Method } \\
\text { D7170 } \\
\text { (FIT) }\end{array}$ & $\begin{array}{l}\text { Other } \\
\text { Ignition } \\
\text { Delay } \\
\text { Method }\end{array}$ & Blend & $\begin{array}{l}\text { Unknown } \\
\text { Method }\end{array}$ & Ref. & Notes \\
\hline \multirow[t]{4}{*}{ benzene } & \multirow[t]{4}{*}{ C6H6 } & \multirow[t]{4}{*}{$71-43-2$} & 14.3 & & & & & & 9 & [35] \\
\hline & & & & & & & 15 & & 1 & $\begin{array}{c}{[1],[23],} \\
{[30]}\end{array}$ \\
\hline & & & & & & & 0 & & 3 & \\
\hline & & & & & & & & -10 & 3,6 & [11] \\
\hline \multirow[t]{4}{*}{ toluene } & \multirow[t]{4}{*}{$\mathrm{C} 7 \mathrm{H} 8$} & \multirow[t]{4}{*}{$108-88-3$} & & & & & 3 & & 1 & $\begin{array}{c}{[1],} \\
{[23],} \\
{[30]}\end{array}$ \\
\hline & & & & & & & -5 & & 3 & \\
\hline & & & & & & & 0 & & 58 & {$[6],[34]$} \\
\hline & & & & 6.0 & & & & & 77 & new \\
\hline \multirow[t]{2}{*}{ 1,2-dimethylbenzene } & \multirow[t]{2}{*}{$\mathrm{C} 8 \mathrm{H} 10$} & \multirow[t]{2}{*}{$95-47-6$} & 8.3 & & & & & & 9 & [35] \\
\hline & & & & 8.3 & & & & & 77 & new \\
\hline \multirow[t]{2}{*}{ 1,3-dimethylbenzene } & \multirow[t]{2}{*}{$\mathrm{C} 8 \mathrm{H} 10$} & \multirow[t]{2}{*}{$108-38-3$} & & & & & -1 & & 1 & $\begin{array}{c}{[1],[23],} \\
{[30]}\end{array}$ \\
\hline & & & & 7.0 & & & & & 77 & new \\
\hline \multirow[t]{2}{*}{ 1,4-dimethylbenzene } & \multirow[t]{2}{*}{$\mathrm{C} 8 \mathrm{H} 10$} & \multirow[t]{2}{*}{$106-42-3$} & & & & & -4 & & 1 & $\begin{array}{c}{[1],[23],} \\
{[30]}\end{array}$ \\
\hline & & & & 6.2 & & & & & 77 & new \\
\hline \multirow[t]{2}{*}{ ethyl benzene } & \multirow[t]{2}{*}{$\mathrm{C} 8 \mathrm{H} 10$} & \multirow[t]{2}{*}{$100-41-4$} & & & & & 4 & & 1 & $\begin{array}{c}{[1],[23],} \\
{[30]}\end{array}$ \\
\hline & & & & 6.3 & & & & & 77 & new \\
\hline indan & $\mathrm{C9H} 10$ & $496-11-7$ & & 8.6 & & & & & 77 & new \\
\hline 1,2,3-trimethylbenzene & $\mathrm{C} 9 \mathrm{H} 12$ & $526-73-8$ & & 10.1 & & & & & 77 & new \\
\hline
\end{tabular}




\begin{tabular}{|c|c|c|c|c|c|c|c|c|c|c|}
\hline Compound & Formula & CAS & $\begin{array}{c}\text { ASTM } \\
\text { Method } \\
\text { D613 } \\
\text { (CFR) }\end{array}$ & $\begin{array}{c}\text { ASTM } \\
\text { Method } \\
\text { D6890 } \\
\text { (IQT) }\end{array}$ & $\begin{array}{c}\text { ASTM } \\
\text { Method } \\
\text { D7170 } \\
\text { (FIT) }\end{array}$ & $\begin{array}{l}\text { Other } \\
\text { Ignition } \\
\text { Delay } \\
\text { Method }\end{array}$ & Blend & $\begin{array}{l}\text { Unknown } \\
\text { Method }\end{array}$ & Ref. & Notes \\
\hline \multirow[t]{2}{*}{ 1,2,4-trimethylbenzene } & \multirow[t]{2}{*}{$\mathrm{C} 9 \mathrm{H} 12$} & \multirow[t]{2}{*}{$95-63-6$} & & 8.9 & & & & & 41 & \\
\hline & & & & 8.9 & & & & & 77 & new \\
\hline \multirow[t]{2}{*}{ 1,3,5-trimethylbenzene } & \multirow[t]{2}{*}{$\mathrm{C9H} 12$} & \multirow[t]{2}{*}{$108-67-8$} & & & & & 8 & & 63 & {$[6],[28]$} \\
\hline & & & & 8.0 & & & & & 77 & new \\
\hline isopropyl benzene & $\mathrm{C} 9 \mathrm{H} 12$ & $98-98-8$ & & & & & 7 & & 1 & $\begin{array}{c}{[1],[23],} \\
{[30]}\end{array}$ \\
\hline n-propylbenzene & $\mathrm{C} 9 \mathrm{H} 12$ & $103-65-1$ & & & & & 16 & & 63 & {$[6],[28]$} \\
\hline $\begin{array}{l}\text { benzaldehyde dimethyl } \\
\text { acetal }\end{array}$ & $\mathrm{C} 9 \mathrm{H} 12 \mathrm{O} 2$ & $1125-88-8$ & & 10.4 & & & & & 76 & new \\
\hline $\begin{array}{l}\text { a-methyl-trans- } \\
\text { cinnamaldehyde }\end{array}$ & $\mathrm{C} 9 \mathrm{H} 8 \mathrm{O}$ & $14371-10-9$ & & 19.1 & & & & & 76 & new \\
\hline \multirow[t]{4}{*}{ tetralin } & \multirow[t]{4}{*}{$\mathrm{C} 10 \mathrm{H} 12$} & \multirow[t]{4}{*}{$119-64-2$} & & 8.9 & & & & & 41 & \\
\hline & & & & 21.3 & & & & & 55 & \\
\hline & & & & & & & 15 & & 1 & $\begin{array}{c}\text { [1],[23], } \\
\text { [30] }\end{array}$ \\
\hline & & & & 9.4 & & & & & 77 & new \\
\hline $\begin{array}{c}1,2,4,5- \\
\text { tetramethylbenzene }\end{array}$ & $\mathrm{C} 10 \mathrm{H} 14$ & $488-23-3$ & & & & & 1 & & 1 & $\begin{array}{c}{[1],[23],} \\
{[30]}\end{array}$ \\
\hline 1,3-diethylbenzene & $\mathrm{C} 10 \mathrm{H} 14$ & $141-93-5$ & & & & & 5 & & 1 & $\begin{array}{c}\text { [1],[23], } \\
{[30]}\end{array}$ \\
\hline $\begin{array}{l}\text { 1-methyl-4- } \\
\text { isopropylbenzene }\end{array}$ & $\mathrm{C} 10 \mathrm{H} 14$ & $99-87-8$ & & & & & 4 & & 1 & $\begin{array}{c}{[1],[23],} \\
{[30]}\end{array}$ \\
\hline \multirow[t]{2}{*}{ n-butylbenzene } & \multirow[t]{2}{*}{$\mathrm{C} 10 \mathrm{H} 14$} & \multirow[t]{2}{*}{$104-51-8$} & & 12.0 & & & & & 41 & \\
\hline & & & & 12.9 & & & & & 76 & new \\
\hline sec-butylbenzene & $\mathrm{C} 10 \mathrm{H} 14$ & $135-98-8$ & & & & & 6 & & 1 & $\begin{array}{c}{[1],[23],} \\
{[30]}\end{array}$ \\
\hline
\end{tabular}




\begin{tabular}{|c|c|c|c|c|c|c|c|c|c|c|}
\hline Compound & Formula & CAS & $\begin{array}{c}\text { ASTM } \\
\text { Method } \\
\text { D613 } \\
\text { (CFR) }\end{array}$ & $\begin{array}{l}\text { ASTM } \\
\text { Method } \\
\text { D6890 } \\
\text { (IQT) }\end{array}$ & $\begin{array}{l}\text { ASTM } \\
\text { Method } \\
\text { D7170 } \\
\text { (FIT) }\end{array}$ & $\begin{array}{l}\text { Other } \\
\text { Ignition } \\
\text { Delay } \\
\text { Method }\end{array}$ & Blend & $\begin{array}{l}\text { Unknown } \\
\text { Method }\end{array}$ & Ref. & Notes \\
\hline tert-butyl benzene & $\mathrm{C} 10 \mathrm{H} 14$ & $98-06-8$ & & & & & 0 & & 1 & $\begin{array}{c}{[1],[23],} \\
{[30]}\end{array}$ \\
\hline naphthalene & $\mathrm{C} 10 \mathrm{H} 8 \mathrm{O}$ & $91-20-3$ & & & & & 22 & & 1 & $\begin{array}{c}{[26],[30} \\
],[37]\end{array}$ \\
\hline \multirow[t]{3}{*}{ 1-methylnaphthalene } & \multirow[t]{3}{*}{$\mathrm{C} 11 \mathrm{H} 10$} & \multirow[t]{3}{*}{$90-12-0$} & 0.0 & & & & & & PRF & [9] \\
\hline & & & & & & & -4 & & 1 & $\begin{array}{c}{[1],[23],} \\
{[30]}\end{array}$ \\
\hline & & & & & & & & 0 & $3,6,7$ & \\
\hline 2-methylnaphthalene & $\mathrm{C} 11 \mathrm{H} 10$ & $91-57-6$ & & & & & 6 & & 1 & $\begin{array}{c}{[1],} \\
{[30],} \\
{[37]}\end{array}$ \\
\hline \multirow[t]{3}{*}{ n-pentylbenzene } & \multirow[t]{3}{*}{$\mathrm{C} 11 \mathrm{H} 16$} & \multirow[t]{3}{*}{$538-68-1$} & & & & & 18 & & 3 & [35] \\
\hline & & & & & & & & 9 & 3 & [8] \\
\hline & & & & & & & & 8 & 7 & \\
\hline \multirow[t]{3}{*}{ biphenyl } & \multirow[t]{3}{*}{$\mathrm{C} 12 \mathrm{H} 10$} & \multirow[t]{3}{*}{$92-52-4$} & & & & & 12 & & 1 & $\begin{array}{c}{[26],[30} \\
\text { ], [37] }\end{array}$ \\
\hline & & & & & & & 21 & & 3 & [35] \\
\hline & & & & & & & & 21 & 7 & \\
\hline $\begin{array}{c}2,6- \\
\text { dimethylnaphthalene }\end{array}$ & $\mathrm{C} 12 \mathrm{H} 12$ & $581-42-0$ & & & & & -7 & & 1 & $\begin{array}{c}{[1],[23],} \\
{[30]}\end{array}$ \\
\hline 1,3,5-triethylbenzene & $\mathrm{C} 12 \mathrm{H} 18$ & $102-25-0$ & & 7.6 & & & & & 77 & new \\
\hline \multirow[t]{2}{*}{ m-diisopropylbenzene } & \multirow[t]{2}{*}{$\mathrm{C} 12 \mathrm{H} 18$} & \multirow[t]{2}{*}{$99-62-7$} & -3.0 & & & & & & 3 & [27] \\
\hline & & & & & & & -12 & & 3 & \\
\hline \multirow[t]{2}{*}{ n-hexylbenzene } & \multirow[t]{2}{*}{ C12H18 } & \multirow[t]{2}{*}{$1077-16-3$} & & & & & & 26 & 2 & \\
\hline & & & & & & & & 26 & 3 & [8] \\
\hline
\end{tabular}




\begin{tabular}{|c|c|c|c|c|c|c|c|c|c|c|}
\hline Compound & Formula & CAS & $\begin{array}{c}\text { ASTM } \\
\text { Method } \\
\text { D613 } \\
\text { (CFR) }\end{array}$ & $\begin{array}{c}\text { ASTM } \\
\text { Method } \\
\text { D6890 } \\
\text { (IQT) }\end{array}$ & $\begin{array}{c}\text { ASTM } \\
\text { Method } \\
\text { D7170 } \\
\text { (FIT) }\end{array}$ & $\begin{array}{l}\text { Other } \\
\text { Ignition } \\
\text { Delay } \\
\text { Method }\end{array}$ & Blend & $\begin{array}{l}\text { Unknown } \\
\text { Method }\end{array}$ & Ref. & Notes \\
\hline diphenylmethane & $\mathrm{C} 13 \mathrm{H} 12$ & $101-81-5$ & & & & & 11 & & 3,4 & [8] \\
\hline n-propyltetralin & $\mathrm{C} 13 \mathrm{H} 18$ & & & & & & & 8 & 3,4 & {$[8]$} \\
\hline \multirow[t]{3}{*}{ n-heptylbenzene } & \multirow[t]{3}{*}{$\mathrm{C} 13 \mathrm{H} 20$} & \multirow[t]{3}{*}{ 1078-71-3 } & & & & & & 35 & 2 & \\
\hline & & & & & & & & 34 & 3 & \\
\hline & & & & & & & & 35 & 3 & [8] \\
\hline 1,2-diphenylethane & $\mathrm{C} 14 \mathrm{H} 14$ & $103-29-7$ & 1.0 & & & & & & 3,4 & {$[27]$} \\
\hline dibenzyl ether & $\mathrm{C} 14 \mathrm{H} 14 \mathrm{O}$ & $103-50-4$ & & 8.1 & & & & & 76 & new \\
\hline 1-butylnaphthalene & $\mathrm{C} 14 \mathrm{H} 16$ & $1634-09-9$ & & & & & & 6 & 7 & \\
\hline 1-n-butylnaphthalene & $\mathrm{C} 14 \mathrm{H} 16$ & $1634-09-9$ & & & & & & 6 & 3,4 & [8] \\
\hline $\begin{array}{l}\text { 2-(1,1-dimethylethyl)- } \\
\text { naphthalene }\end{array}$ & $\mathrm{C} 14 \mathrm{H} 16$ & $2876-35-9$ & & & & & & 3 & 3,4 & [8] \\
\hline ?cis-n-butyltetralin & $\mathrm{C} 14 \mathrm{H} 20$ & & & & & & & 18 & 3,4 & [8] \\
\hline ?trans-n-butyltetralin & $\mathrm{C} 14 \mathrm{H} 20$ & & & & & & & 14 & 3 & \\
\hline sec-butyltetralin & $\mathrm{C} 14 \mathrm{H} 20$ & & & & & & & 7 & 3,4 & [8] \\
\hline tert-butyltetralin & $\mathrm{C} 14 \mathrm{H} 20$ & & & & & & & 17 & 3,4 & [8] \\
\hline 2-phenyloctane & $\mathrm{C} 14 \mathrm{H} 22$ & $777-22-0$ & & & & & & 33 & 3,4 & {$[8]$} \\
\hline \multirow[t]{2}{*}{ n-octylbenzene } & \multirow[t]{2}{*}{$\mathrm{C} 14 \mathrm{H} 22$} & \multirow[t]{2}{*}{$2189-60-8$} & & & & & & 43 & 2 & \\
\hline & & & & & & & & 32 & 3,4 & [8] \\
\hline $\begin{array}{c}1,3,5- \\
\text { triisopropylbenzene }\end{array}$ & $\mathrm{C} 15 \mathrm{H} 24$ & $717-74-8$ & & 2.8 & & & & & 41 & [12] \\
\hline \multirow[t]{2}{*}{ n-nonylbenzene } & \multirow[t]{2}{*}{$\mathrm{C} 15 \mathrm{H} 24$} & \multirow[t]{2}{*}{$1081-77-2$} & & & & & & 50 & 7 & \\
\hline & & & & & & & & 50 & 3,4 & [8] \\
\hline n-octylxylene & $\mathrm{C} 16 \mathrm{H} 26$ & & & & & & & 20 & 3,4 & [8] \\
\hline
\end{tabular}




\begin{tabular}{|c|c|c|c|c|c|c|c|c|c|c|}
\hline Compound & Formula & CAS & $\begin{array}{l}\text { ASTM } \\
\text { Method } \\
\text { D613 } \\
\text { (CFR) }\end{array}$ & $\begin{array}{l}\text { ASTM } \\
\text { Method } \\
\text { D6890 } \\
\text { (IQT) }\end{array}$ & $\begin{array}{l}\text { ASTM } \\
\text { Method } \\
\text { D7170 } \\
\text { (FIT) }\end{array}$ & $\begin{array}{l}\text { Other } \\
\text { Ignition } \\
\text { Delay } \\
\text { Method }\end{array}$ & Blend & $\begin{array}{l}\text { Unknown } \\
\text { Method }\end{array}$ & Ref. & Notes \\
\hline $\begin{array}{l}\text { 2-methyl-2-(beta- } \\
\text { naphthyl)hexane }\end{array}$ & $\mathrm{C} 17 \mathrm{H} 22$ & & & & & & & 10 & 3,4 & [8] \\
\hline 2-phenyl-2-undecene & $\mathrm{C} 17 \mathrm{H} 26$ & & & & & & & 23 & 3,4 & [8] \\
\hline 2-phenylundecane & $\mathrm{C} 17 \mathrm{H} 28$ & $4536-88-3$ & & & & & & 51 & 3,4 & [8] \\
\hline \multirow[t]{2}{*}{ 2-octylnaphthalene } & \multirow[t]{2}{*}{$\mathrm{C} 18 \mathrm{H} 24$} & \multirow[t]{2}{*}{$2876-44-0$} & & & & & & 18 & 7 & \\
\hline & & & & & & & & 18 & 3,4 & [8] \\
\hline $\begin{array}{c}\text { 4-methyl-4-(2- } \\
\text { naphthyl)heptane }\end{array}$ & $\mathrm{C} 18 \mathrm{H} 24$ & & & & & & & 9 & 3,4 & [8] \\
\hline n-octyltetralin & $\mathrm{C} 18 \mathrm{H} 28$ & & & & & & & 18 & 3,4 & [8] \\
\hline 4-phenyldodecane & $\mathrm{C} 18 \mathrm{H} 30$ & $2719-64-4$ & & & & & & 42 & 3,4 & [8] \\
\hline n-dodecylbenzene & $\mathrm{C} 18 \mathrm{H} 30$ & $123-01-3$ & & & & & & 68 & 3,4 & [8] \\
\hline 7-phenyltridecane & $\mathrm{C} 19 \mathrm{H} 32$ & $2400-01-3$ & & & & & & 41 & 3,4 & [8] \\
\hline $\begin{array}{c}\text { 3,6-dimethyl-3-(beta- } \\
\text { naphthyl)octane }\end{array}$ & $\mathrm{C} 2 \mathrm{OH} 28$ & & & & & & & 18 & 3,4 & [8] \\
\hline $\begin{array}{l}\text { 5-methyl-5-(beta- } \\
\text { naphthyl)nonane }\end{array}$ & $\mathrm{C} 2 \mathrm{OH} 28$ & & & & & & & 12 & 3,4 & [8] \\
\hline 2-phenyltetradecane & $\mathrm{C} 2 \mathrm{OH} 34$ & $4534-59-2$ & & & & & & 49 & 3,4 & [8] \\
\hline \multirow[t]{2}{*}{ n-tetradecylbenzene } & \multirow[t]{2}{*}{$\mathrm{C} 2 \mathrm{OH} 34$} & \multirow[t]{2}{*}{$1459-10-5$} & & & & & & 72 & 3 & [8] \\
\hline & & & & & & & & 72 & 7 & \\
\hline $\begin{array}{l}\text { 2-methyl-2-(beta- } \\
\text { naphthyl)decane }\end{array}$ & $\mathrm{C} 21 \mathrm{H} 30$ & & & & & & & 18 & 3,4 & [8] \\
\hline $\begin{array}{l}\text { 3-ethyl-3-(beta- } \\
\text { naphthyl)nonane }\end{array}$ & $\mathrm{C} 21 \mathrm{H} 30$ & & & & & & & 13 & 3,4 & [8] \\
\hline $\begin{array}{l}\text { 2-methyl-2- } \\
\text { phenylpentadecane }\end{array}$ & $\mathrm{C} 22 \mathrm{H} 38$ & 29138-94-1 & & & & & & 39 & 3,4 & [8] \\
\hline
\end{tabular}




\begin{tabular}{|c|c|c|c|c|c|c|c|c|c|c|}
\hline Compound & Formula & CAS & $\begin{array}{c}\text { ASTM } \\
\text { Method } \\
\text { D613 } \\
\text { (CFR) }\end{array}$ & $\begin{array}{l}\text { ASTM } \\
\text { Method } \\
\text { D6890 } \\
\text { (IQT) }\end{array}$ & $\begin{array}{c}\text { ASTM } \\
\text { Method } \\
\text { D7170 } \\
\text { (FIT) }\end{array}$ & $\begin{array}{l}\text { Other } \\
\text { Ignition } \\
\text { Delay } \\
\text { Method }\end{array}$ & Blend & $\begin{array}{l}\text { Unknown } \\
\text { Method }\end{array}$ & Ref. & Notes \\
\hline $\begin{array}{c}\text { 2-methyl-4-isobutyl-4- } \\
\text { phenylundecane }\end{array}$ & $\mathrm{C} 22 \mathrm{H} 38$ & & & & & & & 18 & 3,4 & [8] \\
\hline $\begin{array}{l}\text { 2-methyl-2- } \\
\text { phenylheptadecane }\end{array}$ & $\mathrm{C} 24 \mathrm{H} 42$ & & & & & & & 39 & 3,4 & [8] \\
\hline $\begin{array}{c}\text { 5-butyl-5- } \\
\text { phenyltetradecane }\end{array}$ & $\mathrm{C} 24 \mathrm{H} 42$ & & & & & & & 58 & 3,4 & [8] \\
\hline $\begin{array}{c}\text { 1,2,4-trimethyl-5- } \\
\text { hexadecylbenzene }\end{array}$ & $\mathrm{C} 25 \mathrm{H} 44$ & & & & & & & 42 & 3,4 & [8] \\
\hline di-n-octyltetralin & $\mathrm{C} 26 \mathrm{H} 44$ & & & & & & & 26 & 3,4 & [8] \\
\hline 5-phenyleicosane & $\mathrm{C} 26 \mathrm{H} 46$ & $2400-04-6$ & & & & & & 39 & 3,4 & [8] \\
\hline
\end{tabular}




\section{A.4 Alcohols}

\begin{tabular}{|c|c|c|c|c|c|c|c|c|c|c|}
\hline Compound & Formula & CAS & $\begin{array}{c}\text { ASTM } \\
\text { Method } \\
\text { D613 } \\
\text { (CFR) }\end{array}$ & $\begin{array}{c}\text { ASTM } \\
\text { Method } \\
\text { D6890 } \\
\text { (IQT) }\end{array}$ & $\begin{array}{c}\text { ASTM } \\
\text { Method } \\
\text { D7170 } \\
\text { (FIT) }\end{array}$ & $\begin{array}{l}\text { Other } \\
\text { Ignition } \\
\text { Delay } \\
\text { Method }\end{array}$ & Blend & $\begin{array}{l}\text { Unknown } \\
\text { Method }\end{array}$ & Ref. & Notes \\
\hline \multirow[t]{3}{*}{ methanol } & \multirow[t]{3}{*}{$\mathrm{CH} 4 \mathrm{O}$} & \multirow[t]{3}{*}{$67-56-1$} & & & & & & 5 & 15 & \\
\hline & & & & & & & & 2 & 37 & \\
\hline & & & & & & & & 3 & 13,14 & \\
\hline \multirow[t]{5}{*}{ ethanol } & \multirow[t]{5}{*}{$\mathrm{C} 2 \mathrm{H} 6 \mathrm{O}$} & \multirow[t]{5}{*}{$64-17-5$} & & & & 12 & & & 17 & \\
\hline & & & & & & & 2 & & 57 & [6] \\
\hline & & & & & & & & 2 & 16 & \\
\hline & & & & & & & & 11 & 36 & \\
\hline & & & & & & & & 8 & 13,47 & \\
\hline n-propanol & $\mathrm{C} 3 \mathrm{H} 8 \mathrm{O}$ & $71-23-8$ & & & & & & 12 & 36 & \\
\hline 2-butanol & $\mathrm{C} 4 \mathrm{H} 10 \mathrm{O}$ & $78-92-2$ & & 8.5 & & & & & 57 & \\
\hline $\begin{array}{c}\text { ethylene glycol vinyl } \\
\text { ether }\end{array}$ & $\mathrm{C} 4 \mathrm{H} 8 \mathrm{O} 2$ & $764-48-7$ & & 14.0 & & & & & 76 & new \\
\hline isobutanol & $\mathrm{C} 4 \mathrm{H} 10 \mathrm{O}$ & $78-83-1$ & & 8.5 & & & & & 57 & \\
\hline \multirow[t]{3}{*}{ n-butanol } & \multirow[t]{3}{*}{$\mathrm{C} 4 \mathrm{H} 10 \mathrm{O}$} & \multirow[t]{3}{*}{$71-36-3$} & & 12.0 & & & & & 57 & \\
\hline & & & & & 3.7 & & & & 65 & \\
\hline & & & & & & & & 17 & 36 & \\
\hline t-butanol & $\mathrm{C} 4 \mathrm{H} 10 \mathrm{O}$ & $75-65-0$ & & & & & 6 & & 57 & [6] \\
\hline $\begin{array}{c}\text { 1-methoxy-2 } \\
\text { propanol }\end{array}$ & $\mathrm{C} 4 \mathrm{H} 10 \mathrm{O} 2$ & $107-98-2$ & & 16.3 & & & & & 76 & new \\
\hline cyclopentanol & $\mathrm{C} 5 \mathrm{H} 10 \mathrm{O}$ & $96-41-3$ & & 9.8 & & & & & 76 & new \\
\hline
\end{tabular}




\begin{tabular}{|c|c|c|c|c|c|c|c|c|c|c|}
\hline Compound & Formula & CAS & $\begin{array}{c}\text { ASTM } \\
\text { Method } \\
\text { D613 } \\
\text { (CFR) }\end{array}$ & $\begin{array}{c}\text { ASTM } \\
\text { Method } \\
\text { D6890 } \\
\text { (IQT) }\end{array}$ & $\begin{array}{c}\text { ASTM } \\
\text { Method } \\
\text { D7170 } \\
\text { (FIT) }\end{array}$ & $\begin{array}{l}\text { Other } \\
\text { Ignition } \\
\text { Delay } \\
\text { Method }\end{array}$ & Blend & $\begin{array}{l}\text { Unknown } \\
\text { Method }\end{array}$ & Ref. & Notes \\
\hline \multirow[t]{2}{*}{ 1-pentanol } & \multirow[t]{2}{*}{$\mathrm{C} 5 \mathrm{H} 12 \mathrm{O}$} & \multirow[t]{2}{*}{$71-41-0$} & 18.2 & & & & & & 18 & \\
\hline & & & & & & & & 20 & 36 & \\
\hline 2-methyl-2-butanol & $\mathrm{C} 5 \mathrm{H} 12 \mathrm{O}$ & $75-85-4$ & & 12.1 & & & & & 76 & new \\
\hline 3-methyl-1-butanol & $\mathrm{C} 5 \mathrm{H} 12 \mathrm{O}$ & $123-51-3$ & & 13.1 & & & & & 76 & new \\
\hline isopentanol & $\mathrm{C} 5 \mathrm{H} 12 \mathrm{O}$ & 6423-06-9 & & 18.4 & & & & & 41 & \\
\hline 2-isopropoxyethanol & $\mathrm{C} 5 \mathrm{H} 12 \mathrm{O} 2$ & $109-59-1$ & & 27.8 & & & & & 76 & new \\
\hline cyclohexanol & $\mathrm{C} 6 \mathrm{H} 12 \mathrm{O}$ & $108-93-0$ & & 16.3 & & & & & 76 & new \\
\hline $\begin{array}{l}\text { 4-hydroxy-4-methyl- } \\
\text { 2-pentanone }\end{array}$ & $\mathrm{C} 6 \mathrm{H} 12 \mathrm{O} 2$ & $123-42-2$ & & 11.5 & & & & & 76 & new \\
\hline 1-hexanol & $\mathrm{C} 6 \mathrm{H} 14 \mathrm{O}$ & $111-27-3$ & 23.3 & & & & & & 18 & \\
\hline $\begin{array}{l}\text { 3-methoxy-3-methyl- } \\
\text { 1-butanol }\end{array}$ & $\mathrm{C} 6 \mathrm{H} 14 \mathrm{O} 2$ & $56539-66-3$ & & 10.0 & & & & & 76 & new \\
\hline \multirow[t]{2}{*}{ 1-heptanol } & \multirow[t]{2}{*}{$\mathrm{C} 7 \mathrm{H} 16 \mathrm{O}$} & \multirow[t]{2}{*}{$111-70-6$} & 29.5 & & & & & & 18 & \\
\hline & & & & 29.0 & & & & & 41 & \\
\hline 2-heptanol & $\mathrm{C} 7 \mathrm{H} 16 \mathrm{O}$ & $543-49-7$ & & 25.0 & & & & & 41 & new \\
\hline 4-heptanol & C7H16O & $589-55-9$ & & 21.0 & & & & & 41 & new \\
\hline guaiacol & С7H8O2 & $90-05-1$ & & 19.3 & & & & & 69 & new \\
\hline 2-phenyl ethanol & $\mathrm{C} 8 \mathrm{H} 10 \mathrm{O}$ & $60-12-8$ & & 8.0 & & & & & 69 & new \\
\hline $\begin{array}{c}1,2 \\
\text { dimethoxybenzene }\end{array}$ & $\mathrm{C} 8 \mathrm{H} 10 \mathrm{O} 2$ & $91-16-7$ & & 17.0 & & & & & 69 & new \\
\hline 4-methyl guaiacol & $\mathrm{C} 8 \mathrm{H} 10 \mathrm{O} 2$ & $93-51-6$ & & 19.8 & & & & & 69 & new \\
\hline 2,6-dimethoxyphenol & $\mathrm{C} 8 \mathrm{H} 10 \mathrm{O} 3$ & $91-10-1$ & & 26.0 & & & & & 69 & new \\
\hline
\end{tabular}




\begin{tabular}{|c|c|c|c|c|c|c|c|c|c|c|}
\hline Compound & Formula & CAS & $\begin{array}{l}\text { ASTM } \\
\text { Method } \\
\text { D613 } \\
\text { (CFR) }\end{array}$ & $\begin{array}{c}\text { ASTM } \\
\text { Method } \\
\text { D6890 } \\
\text { (IQT) }\end{array}$ & $\begin{array}{c}\text { ASTM } \\
\text { Method } \\
\text { D7170 } \\
\text { (FIT) }\end{array}$ & $\begin{array}{l}\text { Other } \\
\text { Ignition } \\
\text { Delay } \\
\text { Method }\end{array}$ & Blend & $\begin{array}{l}\text { Unknown } \\
\text { Method }\end{array}$ & Ref. & Notes \\
\hline \multirow[t]{3}{*}{ 1-octanol } & \multirow[t]{3}{*}{$\mathrm{C} 8 \mathrm{H} 18 \mathrm{O}$} & \multirow[t]{3}{*}{$111-87-5$} & 39.1 & & & & & & 18 & \\
\hline & & & & 33.7 & & & & & 41 & [2] \\
\hline & & & & 33.9 & & & & & 76 & new \\
\hline 2-ethyl-1-hexanol & $\mathrm{C} 8 \mathrm{H} 18 \mathrm{O}$ & $104-76-7$ & & 23.5 & & & & & 41 & \\
\hline 3-octanol & $\mathrm{C} 8 \mathrm{H} 18 \mathrm{O}$ & $589-98-0$ & & 25.1 & & & & & 41 & \\
\hline 4-propyl phenol & $\mathrm{C} 9 \mathrm{H} 12 \mathrm{O}$ & $645-56-7$ & & 8.6 & & & & & 70 & new \\
\hline 4-propylphenol & $\mathrm{C} 9 \mathrm{H} 12 \mathrm{O}$ & $645-56-7$ & & 8.6 & & & & & 69 & new \\
\hline 4-ethyl guaiacol & $\mathrm{C} 9 \mathrm{H} 12 \mathrm{O} 2$ & 2785-89-9 & & 19.6 & & & & & 69 & new \\
\hline $\begin{array}{c}\text { 4-methyl-2,6- } \\
\text { dimethoxyphenol }\end{array}$ & $\mathrm{C} 9 \mathrm{H} 12 \mathrm{O} 3$ & $6638-05-7$ & & 25.0 & & & & & 69 & new \\
\hline 1-nonanol & $\mathrm{C} 9 \mathrm{H} 20 \mathrm{O}$ & $143-08-8$ & 46.2 & & & & & & 18 & \\
\hline 2-nonanol & $\mathrm{C} 9 \mathrm{H} 20 \mathrm{O}$ & $628-99-9$ & & 39.6 & & & & & 41 & \\
\hline 4-nonanol & $\mathrm{C} 9 \mathrm{H} 20 \mathrm{O}$ & $5932-79-6$ & & 28.0 & & & & & 41 & new \\
\hline 4-propylguaiacol & $\mathrm{C} 10 \mathrm{H} 14 \mathrm{O} 2$ & $2785-87-7$ & & 18.0 & & & & & 69 & new \\
\hline \multirow[t]{2}{*}{ geraniol } & \multirow[t]{2}{*}{$\mathrm{C} 10 \mathrm{H} 18 \mathrm{O}$} & \multirow[t]{2}{*}{$106-24-1$} & & 22.0 & & & & & 41 & new \\
\hline & & & & 16.5 & & & & & 76 & new \\
\hline \multirow[t]{2}{*}{ linalool } & \multirow[t]{2}{*}{$\mathrm{C} 10 \mathrm{H} 180$} & \multirow[t]{2}{*}{$78-70-6$} & & 20.0 & & & & & 41 & new \\
\hline & & & & 12.9 & & & & & 76 & new \\
\hline$\beta$-citronellol & $\mathrm{C} 10 \mathrm{H} 20 \mathrm{O}$ & $106-22-9$ & & 25.6 & & & & & 41 & \\
\hline 1-decanol & $\mathrm{C} 10 \mathrm{H} 22 \mathrm{O}$ & $112-30-1$ & 50.3 & & & & & & 18 & \\
\hline $\begin{array}{l}\text { 3,7-dimethyl-1- } \\
\text { octanol }\end{array}$ & $\mathrm{C} 10 \mathrm{H} 22 \mathrm{O}$ & $106-21-8$ & & 29.3 & & & & & 41 & \\
\hline 1-undecanol & $\mathrm{C} 11 \mathrm{H} 24 \mathrm{O}$ & $112-42-5$ & 53.2 & & & & & & 18 & \\
\hline 1-dodecanol & $\mathrm{C} 12 \mathrm{H} 26 \mathrm{O}$ & $112-53-8$ & 63.6 & & & & & & 18 & \\
\hline
\end{tabular}




\begin{tabular}{|c|c|c|c|c|c|c|c|c|c|c|}
\hline Compound & Formula & CAS & $\begin{array}{l}\text { ASTM } \\
\text { Method } \\
\text { D613 } \\
\text { (CFR) }\end{array}$ & $\begin{array}{c}\text { ASTM } \\
\text { Method } \\
\text { D6890 } \\
\text { (IQT) }\end{array}$ & $\begin{array}{c}\text { ASTM } \\
\text { Method } \\
\text { D7170 } \\
\text { (FIT) }\end{array}$ & $\begin{array}{l}\text { Other } \\
\text { Ignition } \\
\text { Delay } \\
\text { Method }\end{array}$ & Blend & $\begin{array}{l}\text { Unknown } \\
\text { Method }\end{array}$ & Ref. & Notes \\
\hline 1-tetradecanol & $\mathrm{C} 14 \mathrm{H} 30 \mathrm{O}$ & $112-72-1$ & 80.8 & & & & & & 18 & \\
\hline farnesol & $\mathrm{C} 15 \mathrm{H} 26 \mathrm{O}$ & $4602-84-0$ & & 24.0 & & & & & 41 & new \\
\hline nerolidol & $\mathrm{C} 15 \mathrm{H} 26 \mathrm{O}$ & $7212-44-4$ & & 19.2 & & & & & 76 & new \\
\hline palmitoleyl alcohol & $\mathrm{C} 16 \mathrm{H} 32 \mathrm{O}$ & $10378-01-5$ & & & & 46 & & & 19 & \\
\hline 1-hexadecanol & $\mathrm{C} 16 \mathrm{H} 34 \mathrm{O}$ & $36653-82-4$ & & & & 68 & & & 19 & \\
\hline linolenyl alcohol & $\mathrm{C} 18 \mathrm{H} 32 \mathrm{O}$ & $506-44-5$ & & & & 41 & & & 19 & \\
\hline linoleyl alcohol & $\mathrm{C} 18 \mathrm{H} 34 \mathrm{O}$ & $506-43-4$ & & & & 44 & & & 19 & \\
\hline oleyl alcohol & $\mathrm{C} 18 \mathrm{H} 36 \mathrm{O}$ & $143-28-2$ & & & & 51 & & & 19 & \\
\hline 1-octadecanol & $\mathrm{C} 18 \mathrm{H} 38 \mathrm{O}$ & $112-92-5$ & & & & 81 & & & 19 & [5] \\
\hline
\end{tabular}




\section{A.5 Aldehydes/Ketones}

\begin{tabular}{|c|c|c|c|c|c|c|c|c|c|c|}
\hline Compound & Formula & CAS & $\begin{array}{c}\text { ASTM } \\
\text { Method } \\
\text { D613 } \\
\text { (CFR) }\end{array}$ & $\begin{array}{c}\text { ASTM } \\
\text { Method } \\
\text { D6890 } \\
\text { (IQT) }\end{array}$ & $\begin{array}{c}\text { ASTM } \\
\text { Method } \\
\text { D7170 } \\
\text { (FIT) }\end{array}$ & $\begin{array}{l}\text { Other } \\
\text { Ignition } \\
\text { Delay } \\
\text { Method }\end{array}$ & Blend & $\begin{array}{l}\text { Unknown } \\
\text { Method }\end{array}$ & Ref. & Notes \\
\hline butanal & $\mathrm{C} 4 \mathrm{H} 8 \mathrm{O}$ & $123-72-8$ & & 41.1 & & & & & 76 & new \\
\hline isobutyraldehyde & $\mathrm{C} 4 \mathrm{H} 8 \mathrm{O}$ & $78-84-2$ & & 21.1 & & & & & 76 & new \\
\hline cyclopentanone & $\mathrm{C} 5 \mathrm{H} 8 \mathrm{O}$ & $120-92-3$ & & 9.0 & & & & & 41 & new \\
\hline \multirow[t]{2}{*}{ 3-pentanone } & \multirow[t]{2}{*}{$\mathrm{C} 5 \mathrm{H} 10 \mathrm{O}$} & \multirow[t]{2}{*}{$96-22-0$} & & 9.7 & & & & & 41 & new \\
\hline & & & & 19.5 & & & & & 76 & new \\
\hline \multirow[t]{2}{*}{ valeraldehyde } & \multirow[t]{2}{*}{$\mathrm{C} 5 \mathrm{H} 10 \mathrm{O}$} & \multirow[t]{2}{*}{$110-62-3$} & & 67.9 & & & & & 41 & new \\
\hline & & & & 62.2 & & & & & 76 & new \\
\hline \multirow[t]{2}{*}{ cyclohexanone } & \multirow[t]{2}{*}{$\mathrm{C} 6 \mathrm{H} 10 \mathrm{O}$} & \multirow[t]{2}{*}{$108-94-1$} & & & & & & 10 & 47 & \\
\hline & & & & 10.4 & & & & & 76 & new \\
\hline $\begin{array}{l}\text { 4-methyl-2- } \\
\text { pentanone }\end{array}$ & $\mathrm{C} 6 \mathrm{H} 12 \mathrm{O}$ & $108-10-1$ & & 12.6 & & & & & 76 & new \\
\hline hexanal & $\mathrm{C} 6 \mathrm{H} 12 \mathrm{O}$ & $66-25-1$ & & 75.2 & & & & & 76 & new \\
\hline $\begin{array}{l}\text { 3-cyclohexene-1- } \\
\text { carboxaldehyde }\end{array}$ & $\mathrm{C} 7 \mathrm{H} 10 \mathrm{O}$ & $100-50-5$ & & 28.1 & & & & & 76 & new \\
\hline cycloheptanone & $\mathrm{C} 7 \mathrm{H} 12 \mathrm{O}$ & $502-42-1$ & & 22.5 & & & & & 76 & new \\
\hline \multirow{2}{*}{$\begin{array}{l}\text { 2,4-dimethyl-3- } \\
\text { pentanone }\end{array}$} & \multirow[t]{2}{*}{$\mathrm{C} 7 \mathrm{H} 14 \mathrm{O}$} & \multirow[t]{2}{*}{$565-80-0$} & & 15.8 & & & & & 76 & new \\
\hline & & & & 13.0 & & & & & 41 & new \\
\hline 2-heptanone & $\mathrm{C} 7 \mathrm{H} 14 \mathrm{O}$ & $110-43-0$ & & 30.0 & & & & & 41 & \\
\hline 2-octanone & $\mathrm{C} 8 \mathrm{H} 16 \mathrm{O}$ & $111-13-7$ & & 36.6 & & & & & 76 & new \\
\hline 3-octanone & $\mathrm{C} 8 \mathrm{H} 16 \mathrm{O}$ & $106-68-3$ & & 36.0 & & & & & 41 & $\begin{array}{c}\text { [3] old } \\
\text { value } \\
\text { was } 35.2\end{array}$ \\
\hline \multirow[t]{2}{*}{ octanal } & \multirow[t]{2}{*}{$\mathrm{C} 8 \mathrm{H} 16 \mathrm{O}$} & \multirow[t]{2}{*}{$124-13-0$} & & 109 & & & & & 41 & new \\
\hline & & & & 102.5 & & & & & 76 & new \\
\hline
\end{tabular}




\begin{tabular}{|c|c|c|c|c|c|c|c|c|c|c|}
\hline Compound & Formula & CAS & $\begin{array}{l}\text { ASTM } \\
\text { Method } \\
\text { D613 } \\
\text { (CFR) }\end{array}$ & $\begin{array}{l}\text { ASTM } \\
\text { Method } \\
\text { D6890 } \\
\text { (IQT) }\end{array}$ & $\begin{array}{c}\text { ASTM } \\
\text { Method } \\
\text { D7170 } \\
\text { (FIT) }\end{array}$ & $\begin{array}{l}\text { Other } \\
\text { Ignition } \\
\text { Delay } \\
\text { Method }\end{array}$ & Blend & $\begin{array}{l}\text { Unknown } \\
\text { Method }\end{array}$ & Ref. & Notes \\
\hline $\begin{array}{l}\text { 3,3,5-trimethyl- } \\
\text { cyclohexanone }\end{array}$ & $\mathrm{C} 9 \mathrm{H} 16 \mathrm{O}$ & $873-94-9$ & & 11.9 & & & & & 76 & new \\
\hline whiskey lactone & $\mathrm{C} 9 \mathrm{H} 16 \mathrm{O} 2$ & $39212-23-2$ & & 27.2 & & & & & 76 & new \\
\hline 2-nonanone & $\mathrm{C} 9 \mathrm{H} 18 \mathrm{O}$ & $821-55-6$ & & 46.1 & & & & & 76 & new \\
\hline 4-nonanone & $\mathrm{C} 9 \mathrm{H} 18 \mathrm{O}$ & $4485-09-0$ & & 43.0 & & & & & 41 & new \\
\hline menthone & $\mathrm{C} 10 \mathrm{H} 18 \mathrm{O}$ & $89-80-5$ & & 20.6 & & & & & 76 & new \\
\hline$\delta$-undecalactone & $\mathrm{C} 10 \mathrm{H} 18 \mathrm{O} 2$ & $705-86-2$ & & 48.6 & & & & & 76 & new \\
\hline$\varepsilon$-decalactone & $\mathrm{C} 10 \mathrm{H} 18 \mathrm{O} 2$ & $5579-78-2$ & & 40.5 & & & & & 76 & new \\
\hline$\gamma$-undecanolactone & $\mathrm{C} 11 \mathrm{H} 20 \mathrm{O} 2$ & $104-67-6$ & & 52.6 & & & & & 76 & new \\
\hline 6-undecanone & $\mathrm{C} 11 \mathrm{H} 22 \mathrm{O}$ & $927-49-1$ & & 49.0 & & & & & 41 & new \\
\hline
\end{tabular}




\section{A.6 Ethers}

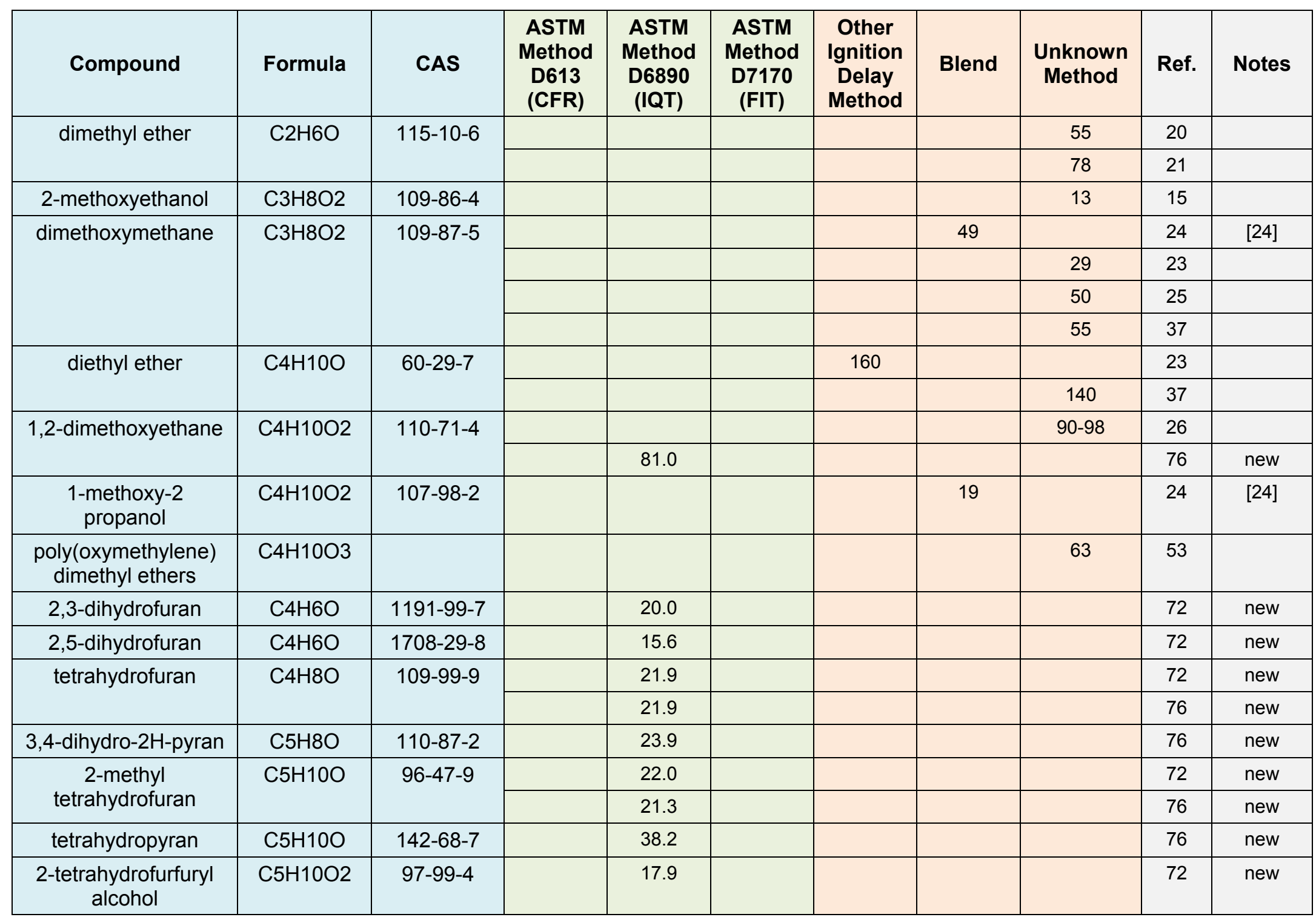




\begin{tabular}{|c|c|c|c|c|c|c|c|c|c|c|}
\hline Compound & Formula & CAS & $\begin{array}{l}\text { ASTM } \\
\text { Method } \\
\text { D613 } \\
\text { (CFR) }\end{array}$ & $\begin{array}{l}\text { ASTM } \\
\text { Method } \\
\text { D6890 } \\
\text { (IQT) }\end{array}$ & $\begin{array}{c}\text { ASTM } \\
\text { Method } \\
\text { D7170 } \\
\text { (FIT) }\end{array}$ & $\begin{array}{l}\text { Other } \\
\text { Ignition } \\
\text { Delay } \\
\text { Method }\end{array}$ & Blend & $\begin{array}{l}\text { Unknown } \\
\text { Method }\end{array}$ & Ref. & Notes \\
\hline $\begin{array}{c}2,2- \\
\text { dimethoxypropane }\end{array}$ & $\mathrm{C} 5 \mathrm{H} 12 \mathrm{O} 2$ & $77-76-9$ & & 31.3 & & & & & 76 & new \\
\hline $\begin{array}{l}\text { diethylene glycol } \\
\text { monomethyl ether }\end{array}$ & $\mathrm{C} 5 \mathrm{H} 12 \mathrm{O} 3$ & $111-77-3$ & & 38.3 & & & & & 76 & new \\
\hline $\begin{array}{l}\text { poly(oxymethylene) } \\
\text { dimethyl ethers }\end{array}$ & $\mathrm{C} 5 \mathrm{H} 12 \mathrm{O} 3$ & & & & & & & 70 & 53 & \\
\hline ethyl acetoacetate & $\mathrm{C} 6 \mathrm{H} 10 \mathrm{O} 3$ & $141-97-9$ & & 13.7 & & & & & 76 & new \\
\hline $\begin{array}{c}\text { cyclopentyl methyl } \\
\text { ether }\end{array}$ & $\mathrm{C} 6 \mathrm{H} 12 \mathrm{O}$ & $5614-37-9$ & & 47.3 & & & & & 76 & new \\
\hline $\begin{array}{c}\text { 2-ethyl } \\
\text { tetrahydrofuran }\end{array}$ & $\mathrm{C} 6 \mathrm{H} 12 \mathrm{O}$ & $1003-30-1$ & & 28.1 & & & & & 72 & new \\
\hline 2-ethoxyethyl acetate & $\mathrm{C} 6 \mathrm{H} 12 \mathrm{O} 3$ & $111-15-9$ & & & & & 40 & & 24 & [24] \\
\hline \multirow[t]{3}{*}{ 1,1-diethoxy ethane } & \multirow[t]{3}{*}{$\mathrm{C} 6 \mathrm{H} 14 \mathrm{O} 2$} & \multirow[t]{3}{*}{$105-57-7$} & & 40.0 & & & & & 71 & new \\
\hline & & & & & & & & 40 & 56 & \\
\hline & & & & 54.1 & & & & & 76 & new \\
\hline \multirow[t]{2}{*}{ 2-butoxyethanol } & \multirow[t]{2}{*}{$\mathrm{C} 6 \mathrm{H} 14 \mathrm{O} 2$} & \multirow[t]{2}{*}{$111-76-2$} & & & & & 41 & & 24 & [24] \\
\hline & & & & & & & & 35 & 27 & \\
\hline diethoxymethane & $\mathrm{C} 6 \mathrm{H} 14 \mathrm{O} 2$ & $462-95-3$ & & 57.3 & & & & & 76 & new \\
\hline \multirow[t]{5}{*}{ 2-methoxyethyl ether } & \multirow[t]{5}{*}{$\mathrm{C} 6 \mathrm{H} 14 \mathrm{O} 3$} & \multirow[t]{5}{*}{$111-96-6$} & & & & 170 & & & 23 & \\
\hline & & & & & & & 109 & & 28 & \\
\hline & & & & & & & & $>100$ & 15 & \\
\hline & & & & & & & & $112-130$ & 26 & {$[16],[23]$} \\
\hline & & & & & & & & 126 & 27 & \\
\hline diisopropyl ether & $\mathrm{C} 6 \mathrm{H} 14 \mathrm{O} 3$ & $108-20-3$ & & 23.6 & & & & & 76 & new \\
\hline $\begin{array}{l}\text { poly(oxymethylene) } \\
\text { dimethyl ethers }\end{array}$ & $\mathrm{C} 6 \mathrm{H} 14 \mathrm{O} 3$ & & & & & & & 90 & 53 & \\
\hline $\begin{array}{c}\text { 2,4,7,9-tetra-oxa- } \\
\text { decane }\end{array}$ & $\mathrm{C} 6 \mathrm{H} 14 \mathrm{O} 4$ & & & & & & 58 & & 24 & {$[15],[24]$} \\
\hline
\end{tabular}




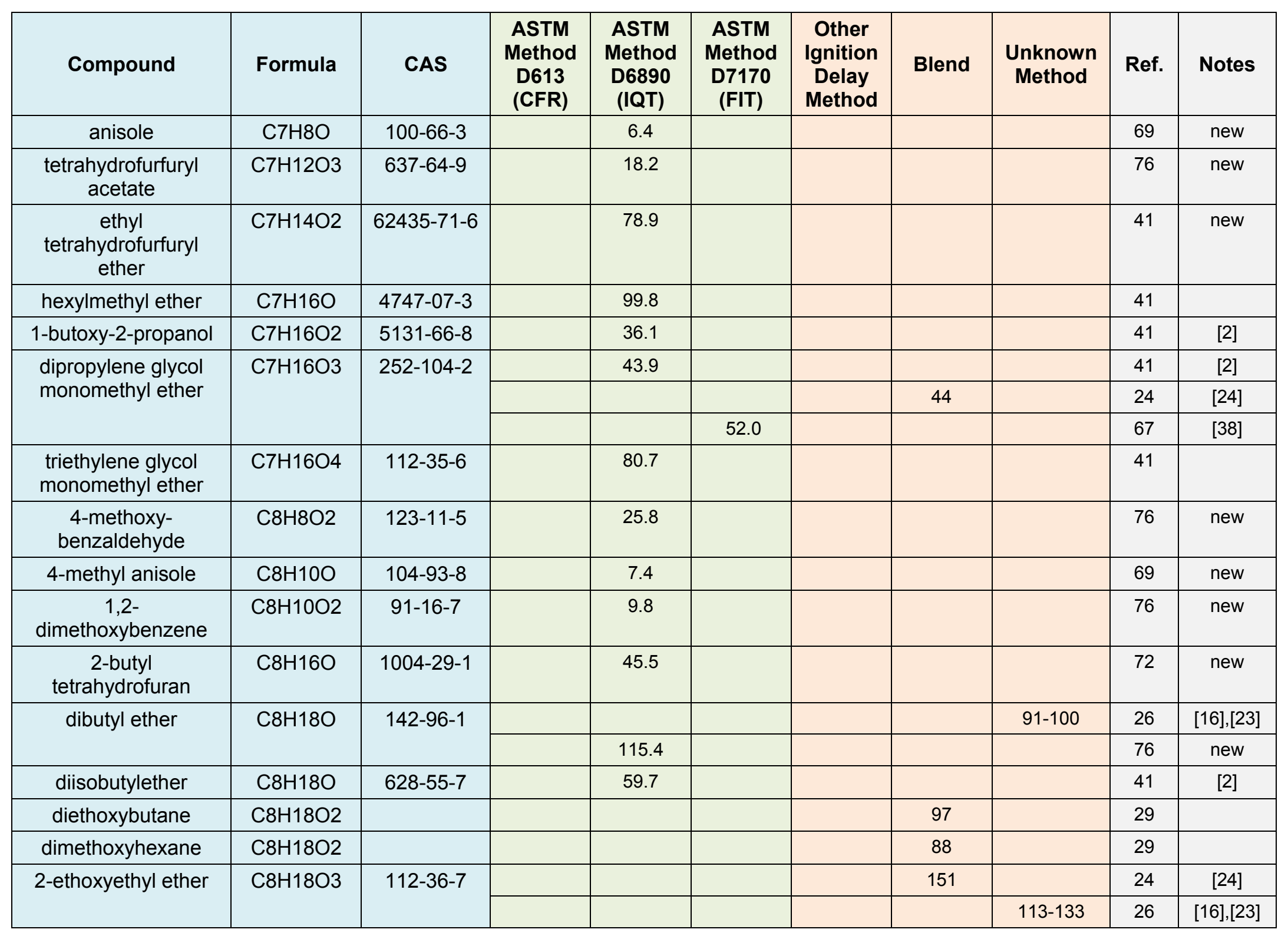




\begin{tabular}{|c|c|c|c|c|c|c|c|c|c|c|}
\hline Compound & Formula & CAS & $\begin{array}{l}\text { ASTM } \\
\text { Method } \\
\text { D613 } \\
\text { (CFR) }\end{array}$ & $\begin{array}{c}\text { ASTM } \\
\text { Method } \\
\text { D6890 } \\
\text { (IQT) }\end{array}$ & $\begin{array}{c}\text { ASTM } \\
\text { Method } \\
\text { D7170 } \\
\text { (FIT) }\end{array}$ & $\begin{array}{l}\text { Other } \\
\text { Ignition } \\
\text { Delay } \\
\text { Method }\end{array}$ & Blend & $\begin{array}{l}\text { Unknown } \\
\text { Method }\end{array}$ & Ref. & Notes \\
\hline $\begin{array}{l}\text { triethyleneglycol } \\
\text { dimethyl ether }\end{array}$ & $\mathrm{C} 8 \mathrm{H} 18 \mathrm{O} 4$ & $112-49-2$ & & & & & & 120 & 47 & \\
\hline dibutoxymethane & $\mathrm{C} 9 \mathrm{H} 20 \mathrm{O} 2$ & $2568-90-3$ & & & & & & 74 & 25 & \\
\hline 4-propylanisole & $\mathrm{C} 10 \mathrm{H} 14 \mathrm{O}$ & $104-45-0$ & & 7.5 & & & & & 69 & new \\
\hline eucalyptol & $\mathrm{C} 10 \mathrm{H} 180$ & $470-82-6$ & & 18.8 & & & & & 76 & new \\
\hline \multirow[t]{2}{*}{ diisoamylether } & \multirow[t]{2}{*}{$\mathrm{C} 10 \mathrm{H} 22 \mathrm{O}$} & \multirow[t]{2}{*}{$544-01-4$} & & 96.3 & & & & & 41 & \\
\hline & & & & 96.0 & & & & & 41 & new \\
\hline \multirow[t]{2}{*}{ dipentyl ether } & \multirow[t]{2}{*}{$\mathrm{C} 10 \mathrm{H} 22 \mathrm{O}$} & \multirow[t]{2}{*}{$693-65-2$} & & & & & & $111-130$ & 26 & {$[16],[23]$} \\
\hline & & & & 111.0 & & & & & 41 & new \\
\hline \multirow{3}{*}{$\begin{array}{l}\text { tripropylene glycol } \\
\text { monomethyl ether }\end{array}$} & \multirow[t]{3}{*}{$\mathrm{C} 10 \mathrm{H} 22 \mathrm{O} 4$} & \multirow[t]{3}{*}{$25498-49-1$} & & 81.3 & & & & & 41 & \\
\hline & & & & & & & 63 & & 24 & {$[15],[24]$} \\
\hline & & & & & & & & 65 & 47 & \\
\hline rose oxide & $\mathrm{C} 10 \mathrm{H} 8 \mathrm{O}$ & $16409-43-1$ & & 30.0 & & & & & 41 & \\
\hline $\begin{array}{c}\text { 1,4-cyclohexane- } \\
\text { dimethanol divinyl } \\
\text { ether }\end{array}$ & $\mathrm{C} 12 \mathrm{H} 20 \mathrm{O} 2$ & $17351-75-6$ & & 61.1 & & & & & 76 & new \\
\hline dodecyl vinyl ether & $\mathrm{C} 14 \mathrm{H} 30 \mathrm{O}$ & & & 101.7 & & & & & 76 & new \\
\hline
\end{tabular}




\section{A.7 Esters}

\section{A.7.1 Esters: Saturated}

\begin{tabular}{|c|c|c|c|c|c|c|c|c|c|c|}
\hline Compound & Formula & CAS & $\begin{array}{c}\text { ASTM } \\
\text { Method } \\
\text { D613 } \\
\text { (CFR) }\end{array}$ & $\begin{array}{c}\text { ASTM } \\
\text { Method } \\
\text { D6890 } \\
\text { (IQT) }\end{array}$ & $\begin{array}{c}\text { ASTM } \\
\text { Method } \\
\text { D7170 } \\
\text { (FIT) }\end{array}$ & $\begin{array}{l}\text { Other } \\
\text { Ignition } \\
\text { Delay } \\
\text { Method }\end{array}$ & Blend & $\begin{array}{l}\text { Unknown } \\
\text { Method }\end{array}$ & Ref. & Notes \\
\hline methyl acetate & $\mathrm{C} 3 \mathrm{H} 6 \mathrm{O} 2$ & $79-20-9$ & & 19.5 & & & & & 76 & new \\
\hline \multirow[t]{2}{*}{ methyl butanoate } & \multirow[t]{2}{*}{$\mathrm{C} 5 \mathrm{H} 10 \mathrm{O} 2$} & \multirow[t]{2}{*}{$623-42-7$} & 6.4 & & & & & & 66 & \\
\hline & & & & & & & 6 & & 64 & [6] \\
\hline methyl levulinate & $\mathrm{C} 6 \mathrm{H} 10 \mathrm{O} 3$ & $624-45-3$ & & 7.8 & & & & & 41 & new \\
\hline methyl pentanoate & $\mathrm{C} 6 \mathrm{H} 12 \mathrm{O} 2$ & $624-24-8$ & & 13.3 & & & & & 58 & \\
\hline $\begin{array}{c}\text { propylene glycol } \\
\text { monomethyl ether } \\
\text { acetate }\end{array}$ & $\mathrm{C} 6 \mathrm{H} 12 \mathrm{O} 3$ & $108-65-6$ & & 24.0 & & & & & 76 & new \\
\hline ethyl levulinate & $\mathrm{C} 7 \mathrm{H} 12 \mathrm{O} 3$ & $539-88-8$ & & 6.0 & & & & & 41 & new \\
\hline \multirow[t]{3}{*}{ methyl caproate } & \multirow[t]{3}{*}{$\mathrm{C} 7 \mathrm{H} 14 \mathrm{O} 2$} & \multirow[t]{3}{*}{$106-70-7$} & 18.0 & & & & & & 18 & \\
\hline & & & & 23.9 & & & & & 41 & \\
\hline & & & & 25.3 & & & & & 76 & new \\
\hline pentyl acetate & $\mathrm{C} 7 \mathrm{H} 14 \mathrm{O} 2$ & $628-63-7$ & & 23.6 & & & & & 41 & new \\
\hline butyl butanoate & $\mathrm{C} 8 \mathrm{H} 16 \mathrm{O} 2$ & $109-21-7$ & & 17.8 & & & & & 41 & \\
\hline \multirow[t]{2}{*}{ ethyl hexanoate } & \multirow[t]{2}{*}{$\mathrm{C} 8 \mathrm{H} 16 \mathrm{O} 2$} & \multirow[t]{2}{*}{ 123-66-0 } & & 27.5 & & & & & 76 & new \\
\hline & & & & 28.0 & & & & & 41 & new \\
\hline hexyl acetate & $\mathrm{C} 8 \mathrm{H} 16 \mathrm{O} 2$ & $142-92-7$ & & 32.2 & & & & & 76 & new \\
\hline n-hexyl acetate & $\mathrm{C} 8 \mathrm{H} 16 \mathrm{O} 2$ & $142-92-7$ & & 33.8 & & & & & 41 & \\
\hline methyl heptanoate & $\mathrm{C} 8 \mathrm{H} 16 \mathrm{O} 2$ & $106-73-0$ & & 34.2 & & & & & 41 & {$[2]$} \\
\hline propyl pentanoate & $\mathrm{C} 8 \mathrm{H} 16 \mathrm{O} 2$ & $141-06-0$ & & 20.7 & & & & & 58 & \\
\hline
\end{tabular}




\begin{tabular}{|c|c|c|c|c|c|c|c|c|c|c|}
\hline Compound & Formula & CAS & $\begin{array}{l}\text { ASTM } \\
\text { Method } \\
\text { D613 } \\
\text { (CFR) }\end{array}$ & $\begin{array}{l}\text { ASTM } \\
\text { Method } \\
\text { D6890 } \\
\text { (IQT) }\end{array}$ & $\begin{array}{l}\text { ASTM } \\
\text { Method } \\
\text { D7170 } \\
\text { (FIT) }\end{array}$ & $\begin{array}{l}\text { Other } \\
\text { Ignition } \\
\text { Delay } \\
\text { Method }\end{array}$ & Blend & $\begin{array}{l}\text { Unknown } \\
\text { Method }\end{array}$ & Ref. & Notes \\
\hline butyl pentanoate & $\mathrm{C} 9 \mathrm{H} 18 \mathrm{O} 2$ & $591-68-4$ & & 23.5 & & & & & 58 & \\
\hline \multirow[t]{3}{*}{ methyl octanoate } & \multirow[t]{3}{*}{$\mathrm{C} 9 \mathrm{H} 18 \mathrm{O} 2$} & \multirow[t]{3}{*}{$111-11-5$} & 33.6 & & & & & & 31 & [37] \\
\hline & & & & & & & & 34 & 22 & \\
\hline & & & & 39.8 & & & & & 68 & new \\
\hline butyl hexanoate & $\mathrm{C} 10 \mathrm{H} 20 \mathrm{O} 2$ & $626-82-4$ & & 31.0 & & & & & 41 & new \\
\hline ethyl octanoate & $\mathrm{C} 10 \mathrm{H} 20 \mathrm{O} 2$ & $106-32-1$ & & 42.2 & & & & & 68 & new \\
\hline hexyl butyrate & $\mathrm{C} 10 \mathrm{H} 20 \mathrm{O} 2$ & $2639-63-6$ & & 29.0 & & & & & 41 & new \\
\hline \multirow[t]{3}{*}{ pentyl pentanoate } & \multirow[t]{3}{*}{$\mathrm{C} 10 \mathrm{H} 20 \mathrm{O} 2$} & \multirow[t]{3}{*}{$2173-56-0$} & & 28.8 & & & & & 41 & \\
\hline & & & & 27.6 & & & & & 58 & \\
\hline & & & & 30.0 & & & & & 70 & new \\
\hline \multirow[t]{8}{*}{ methyl decanoate } & \multirow[t]{8}{*}{$\mathrm{C} 11 \mathrm{H} 22 \mathrm{O} 2$} & \multirow[t]{8}{*}{$110-42-9$} & 47.9 & & & & & & 18 & \\
\hline & & & 47.2 & & & & & & 31 & \\
\hline & & & & 52.7 & & & & & 41 & [7] \\
\hline & & & & 50.7 & & & & & 41 & \\
\hline & & & & 51.6 & & & & & 42 & \\
\hline & & & & 54.1 & & & & & 64 & \\
\hline & & & & & 52.1 & & & & 65 & \\
\hline & & & & 51.6 & & & & & 68 & new \\
\hline butyl octanoate & $\mathrm{C} 12 \mathrm{H} 24 \mathrm{O} 2$ & $589-75-3$ & 39.6 & & & & & & 31 & \\
\hline decyl acetate & $\mathrm{C} 12 \mathrm{H} 24 \mathrm{O} 2$ & $112-17-4$ & & & & & 62 & & 32 & [21],[26] \\
\hline \multirow[t]{3}{*}{ ethyl decanoate } & \multirow[t]{3}{*}{$\mathrm{C} 12 \mathrm{H} 24 \mathrm{O} 2$} & \multirow[t]{3}{*}{$110-38-3$} & 51.2 & & & & & & 31 & \\
\hline & & & & & & & 60 & & 32 & {$[21],[26]$} \\
\hline & & & & 54.6 & & & & & 68 & new \\
\hline hexyl hexanoate & $\mathrm{C} 12 \mathrm{H} 24 \mathrm{O} 2$ & $6378-65-0$ & & 40.0 & & & & & 41 & new \\
\hline
\end{tabular}




\begin{tabular}{|c|c|c|c|c|c|c|c|c|c|c|}
\hline Compound & Formula & CAS & $\begin{array}{l}\text { ASTM } \\
\text { Method } \\
\text { D613 } \\
\text { (CFR) }\end{array}$ & $\begin{array}{l}\text { ASTM } \\
\text { Method } \\
\text { D6890 } \\
\text { (IQT) }\end{array}$ & $\begin{array}{l}\text { ASTM } \\
\text { Method } \\
\text { D7170 } \\
\text { (FIT) }\end{array}$ & $\begin{array}{l}\text { Other } \\
\text { Ignition } \\
\text { Delay } \\
\text { Method }\end{array}$ & Blend & $\begin{array}{l}\text { Unknown } \\
\text { Method }\end{array}$ & Ref. & Notes \\
\hline isopropyl decanoate & $\mathrm{C} 13 \mathrm{H} 26 \mathrm{O} 2$ & $2311-59-3$ & 46.6 & & & & & & 31 & \\
\hline \multirow[t]{8}{*}{ methyl laurate } & \multirow[t]{8}{*}{$\mathrm{C} 13 \mathrm{H} 26 \mathrm{O} 2$} & \multirow[t]{8}{*}{$111-82-0$} & 61.2 & & & & & & 12 & [37] \\
\hline & & & 60.8 & & & & & & 18 & {$[37]$} \\
\hline & & & 61.4 & & & & & & 31 & \\
\hline & & & & 66.7 & & & & & 42 & \\
\hline & & & & 66.3 & & & & & 41 & \\
\hline & & & & & & 54 & & & 24 & \\
\hline & & & & & & & 70 & & 32 & {$[21],[26]$} \\
\hline & & & & 66.7 & & & & & 68 & new \\
\hline octyl valerate & $\mathrm{C} 13 \mathrm{H} 26 \mathrm{O} 2$ & $5451-85-4$ & & & & & 49 & & 32 & {$[21],[26]$} \\
\hline \multirow[t]{2}{*}{ propyl decanoate } & \multirow[t]{2}{*}{$\mathrm{C} 13 \mathrm{H} 26 \mathrm{O} 2$} & \multirow[t]{2}{*}{$30673-60-0$} & 52.9 & & & & & & 31 & \\
\hline & & & & & & & 64 & & 32 & {$[21],[26]$} \\
\hline \multirow[t]{2}{*}{ butyl decanoate } & \multirow[t]{2}{*}{$\mathrm{C} 14 \mathrm{H} 28 \mathrm{O} 2$} & \multirow[t]{2}{*}{$30673-36-0$} & 54.6 & & & & & & 31 & \\
\hline & & & & & & & 63 & & 32 & {$[21],[26]$} \\
\hline dodecyl acetate & $\mathrm{C} 14 \mathrm{H} 28 \mathrm{O} 2$ & $112-66-3$ & & & & & 77 & & 32 & [21], [26] \\
\hline ethyl laurate & $\mathrm{C} 14 \mathrm{H} 28 \mathrm{O} 2$ & $106-33-2$ & & & & & 73 & & 32 & {$[21],[26]$} \\
\hline decyl valerate & $\mathrm{C} 15 \mathrm{H} 30 \mathrm{O} 2$ & $5454-12-6$ & & & & & 61 & & 32 & {$[21],[26]$} \\
\hline \multirow[t]{4}{*}{ methyl myristate } & \multirow[t]{4}{*}{$\mathrm{C} 15 \mathrm{H} 30 \mathrm{O} 2$} & \multirow[t]{4}{*}{$124-10-7$} & 73.5 & & & & & & 18 & \\
\hline & & & 66.2 & & & & & & 31 & \\
\hline & & & & & & & 72 & & 32 & {$[21],[26]$} \\
\hline & & & & 75.8 & & & & & 76 & new \\
\hline propyl laurate & $\mathrm{C} 15 \mathrm{H} 30 \mathrm{O} 2$ & $3681-78-5$ & & & & & 71 & & 32 & {$[21],[26]$} \\
\hline butyl laurate & $\mathrm{C} 16 \mathrm{H} 32 \mathrm{O} 2$ & $106-18-3$ & & & & & 73 & & 32 & {$[21],[26]$} \\
\hline
\end{tabular}




\begin{tabular}{|c|c|c|c|c|c|c|c|c|c|c|}
\hline Compound & Formula & CAS & $\begin{array}{l}\text { ASTM } \\
\text { Method } \\
\text { D613 } \\
\text { (CFR) }\end{array}$ & $\begin{array}{c}\text { ASTM } \\
\text { Method } \\
\text { D6890 } \\
\text { (IQT) }\end{array}$ & $\begin{array}{l}\text { ASTM } \\
\text { Method } \\
\text { D7170 } \\
\text { (FIT) }\end{array}$ & $\begin{array}{l}\text { Other } \\
\text { Ignition } \\
\text { Delay } \\
\text { Method }\end{array}$ & Blend & $\begin{array}{l}\text { Unknown } \\
\text { Method }\end{array}$ & Ref. & Notes \\
\hline \multirow[t]{2}{*}{ ethyl myristate } & \multirow[t]{2}{*}{$\mathrm{C} 16 \mathrm{H} 32 \mathrm{O} 2$} & \multirow[t]{2}{*}{$124-06-1$} & 66.9 & & & & & & 31 & \\
\hline & & & & & & & 72 & & 32 & {$[21],[26]$} \\
\hline hexyl caprate & $\mathrm{C} 16 \mathrm{H} 32 \mathrm{O} 2$ & $10448-26-7$ & & & & & 64 & & 32 & {$[21],[26]$} \\
\hline tetradecyl acetate & $\mathrm{C} 16 \mathrm{H} 32 \mathrm{O} 2$ & $638-59-5$ & & & & & 81 & & 32 & {$[21],[26]$} \\
\hline dodecyl valerate & $\mathrm{C} 17 \mathrm{H} 34 \mathrm{O} 2$ & & & & & & 67 & & 32 & {$[21],[26]$} \\
\hline \multirow[t]{7}{*}{ methyl palmitate } & \multirow[t]{7}{*}{$\mathrm{C} 17 \mathrm{H} 34 \mathrm{O} 2$} & \multirow[t]{7}{*}{$112-39-0$} & 74.5 & & & & & & 31 & [5] \\
\hline & & & 74.3 & & & & & & 12 & [37] \\
\hline & & & 74.3 & & & & & & 18 & [5] \\
\hline & & & & 85.9 & & & & & 42 & [5] \\
\hline & & & & & & 91 & & & 19 & [5] \\
\hline & & & & & & 86 & & & 33 & [5] \\
\hline & & & & & & & 80 & & 32 & $\begin{array}{c}\text { [21], [5], } \\
\text { [26] }\end{array}$ \\
\hline propyl myristate & $\mathrm{C} 17 \mathrm{H} 34 \mathrm{O} 2$ & $14303-70-9$ & & & & & 71 & & 32 & {$[21],[26]$} \\
\hline \multirow[t]{2}{*}{ butyl myristate } & \multirow[t]{2}{*}{$\mathrm{C} 18 \mathrm{H} 36 \mathrm{O} 2$} & \multirow[t]{2}{*}{$110-36-1$} & 69.4 & & & & & & 31 & \\
\hline & & & & & & & 73 & & 32 & {$[21],[26]$} \\
\hline \multirow[t]{2}{*}{ ethyl palmitate } & \multirow[t]{2}{*}{$\mathrm{C} 18 \mathrm{H} 36 \mathrm{O} 2$} & \multirow[t]{2}{*}{$628-97-7$} & & & & 93 & & & 33 & \\
\hline & & & & & & & 80 & & 32 & {$[21],[26]$} \\
\hline hexadecyl acetate & $\mathrm{C} 18 \mathrm{H} 36 \mathrm{O} 2$ & $629-70-9$ & & & & & 86 & & 32 & {$[21],[26]$} \\
\hline hexyl laurate & $\mathrm{C} 18 \mathrm{H} 36 \mathrm{O} 2$ & $34316-64-8$ & & & & & 74 & & 32 & {$[21],[26]$} \\
\hline isopropyl palmitate & $\mathrm{C} 19 \mathrm{H} 38 \mathrm{O} 2$ & $142-91-6$ & & & & 83 & & & 33 & \\
\hline
\end{tabular}




\begin{tabular}{|c|c|c|c|c|c|c|c|c|c|c|}
\hline Compound & Formula & CAS & $\begin{array}{l}\text { ASTM } \\
\text { Method } \\
\text { D613 } \\
\text { (CFR) }\end{array}$ & $\begin{array}{c}\text { ASTM } \\
\text { Method } \\
\text { D6890 } \\
\text { (IQT) }\end{array}$ & $\begin{array}{c}\text { ASTM } \\
\text { Method } \\
\text { D7170 } \\
\text { (FIT) }\end{array}$ & $\begin{array}{l}\text { Other } \\
\text { Ignition } \\
\text { Delay } \\
\text { Method }\end{array}$ & Blend & $\begin{array}{l}\text { Unknown } \\
\text { Method }\end{array}$ & Ref. & Notes \\
\hline \multirow[t]{7}{*}{ methyl stearate } & \multirow[t]{7}{*}{$\mathrm{C} 19 \mathrm{H} 38 \mathrm{O} 2$} & \multirow[t]{7}{*}{$112-61-8$} & 86.9 & & & & & & 12 & [37] \\
\hline & & & 75.6 & & & & & & 18 & \\
\hline & & & & 95.6 & & & & & 41 & [20] \\
\hline & & & & & & 100 & & & 19 & \\
\hline & & & & & & 101 & & & 33 & [23] \\
\hline & & & & & & & 87 & & 31 & {$[29],[31]$} \\
\hline & & & & & & & 81 & & 32 & $\begin{array}{c}{[21],} \\
{[23],[26]}\end{array}$ \\
\hline \multirow[t]{2}{*}{ propyl palmitate } & \multirow[t]{2}{*}{$\mathrm{C} 19 \mathrm{H} 38 \mathrm{O} 2$} & \multirow[t]{2}{*}{$2239-78-2$} & & & & & 83 & & 32 & {$[21],[26]$} \\
\hline & & & & & & 85 & & & 33 & \\
\hline tetradecyl valerate & $\mathrm{C} 19 \mathrm{H} 38 \mathrm{O} 2$ & & & & & & 68 & & 32 & {$[21],[26]$} \\
\hline 2-butyl palmitate & $\mathrm{C} 2 \mathrm{OH} 40 \mathrm{O} 2$ & & & & & 85 & & & 33 & \\
\hline \multirow[t]{2}{*}{ butyl palmitate } & \multirow[t]{2}{*}{$\mathrm{C} 2 \mathrm{OH} 40 \mathrm{O} 2$} & \multirow[t]{2}{*}{$111-06-8$} & & & & 92 & & & 33 & \\
\hline & & & & & & & 87 & & 32 & {$[21],[26]$} \\
\hline decyl caprate & $\mathrm{C} 2 \mathrm{OH} 40 \mathrm{O} 2$ & $1654-86-0$ & & & & & 81 & & 32 & {$[21],[26]$} \\
\hline \multirow[t]{4}{*}{ ethyl stearate } & \multirow[t]{4}{*}{$\mathrm{C} 2 \mathrm{OH} 40 \mathrm{O} 2$} & \multirow[t]{4}{*}{$111-61-5$} & 76.8 & & & & & & 12 & {$[37]$} \\
\hline & & & & & & & 86 & & 32 & {$[21],[26]$} \\
\hline & & & & & & 98 & & & 33 & \\
\hline & & & & & & 77 & & & 34 & \\
\hline hexyl myristate & $\mathrm{C} 2 \mathrm{OH} 40 \mathrm{O} 2$ & $42231-99-2$ & & & & & 72 & & 32 & {$[21],[26]$} \\
\hline isobutyl palmitate & $\mathrm{C} 2 \mathrm{OH} 40 \mathrm{O} 2$ & $110-34-9$ & & & & 84 & & & 33 & \\
\hline octadecyl acetate & $\mathrm{C} 2 \mathrm{OH} 40 \mathrm{O} 2$ & $822-23-1$ & & & & & 90 & & 32 & {$[21],[26]$} \\
\hline octyl laurate & $\mathrm{C} 2 \mathrm{OH} 40 \mathrm{O} 2$ & $5303-24-2$ & & & & & 84 & & 32 & {$[21],[26]$} \\
\hline hexadecyl valerate & $\mathrm{C} 21 \mathrm{H} 42 \mathrm{O} 2$ & $125164-54-7$ & & & & & 70 & & 32 & {$[21],[26]$} \\
\hline
\end{tabular}




\begin{tabular}{|c|c|c|c|c|c|c|c|c|c|c|}
\hline Compound & Formula & CAS & $\begin{array}{l}\text { ASTM } \\
\text { Method } \\
\text { D613 } \\
\text { (CFR) }\end{array}$ & $\begin{array}{l}\text { ASTM } \\
\text { Method } \\
\text { D6890 } \\
\text { (IQT) }\end{array}$ & $\begin{array}{l}\text { ASTM } \\
\text { Method } \\
\text { D7170 } \\
\text { (FIT) }\end{array}$ & $\begin{array}{l}\text { Other } \\
\text { Ignition } \\
\text { Delay } \\
\text { Method }\end{array}$ & Blend & $\begin{array}{l}\text { Unknown } \\
\text { Method }\end{array}$ & Ref. & Notes \\
\hline isopropyl stearate & $\mathrm{C} 21 \mathrm{H} 42 \mathrm{O} 2$ & $112-10-7$ & & & & 97 & & & 33 & \\
\hline methyl arachidate & $\mathrm{C} 21 \mathrm{H} 42 \mathrm{O} 2$ & $1120-28-1$ & & & & 100 & & & 19 & \\
\hline \multirow[t]{2}{*}{ propyl stearate } & \multirow[t]{2}{*}{$\mathrm{C} 21 \mathrm{H} 42 \mathrm{O} 2$} & \multirow[t]{2}{*}{$3634-92-2$} & & & & 91 & & & 33 & \\
\hline & & & & & & 70 & & & 34 & \\
\hline 2-butyl stearate & $\mathrm{C} 22 \mathrm{H} 44 \mathrm{O} 2$ & $123-95-5$ & & & & 98 & & & 33 & \\
\hline \multirow[t]{2}{*}{ butyl stearate } & \multirow[t]{2}{*}{$\mathrm{C} 22 \mathrm{H} 44 \mathrm{O} 2$} & \multirow[t]{2}{*}{$123-95-5$} & & & & 93 & & & 33 & \\
\hline & & & & & & 80 & & & 34 & \\
\hline decyl laurate & $\mathrm{C} 22 \mathrm{H} 44 \mathrm{O} 2$ & $36528-28-6$ & & & & & 84 & & 32 & {$[21],[26]$} \\
\hline hexyl palmitate & $\mathrm{C} 22 \mathrm{H} 44 \mathrm{O} 2$ & $42232-25-7$ & & & & & 87 & & 32 & [21],[26] \\
\hline isobutyl stearate & $\mathrm{C} 22 \mathrm{H} 44 \mathrm{O} 2$ & $646-13-9$ & & & & 99 & & & 33 & \\
\hline octyl myristate & $\mathrm{C} 22 \mathrm{H} 44 \mathrm{O} 2$ & $16260-26-7$ & & & & & 71 & & 32 & {$[21],[26]$} \\
\hline \multirow{2}{*}{$\begin{array}{l}\text { 2-ethylhexyl } \\
\text { palmitate }\end{array}$} & \multirow[t]{2}{*}{$\mathrm{C} 24 \mathrm{H} 48 \mathrm{O} 2$} & \multirow[t]{2}{*}{$16958-85-3$} & & & & & 107 & & 32 & {$[21],[26]$} \\
\hline & & & & & & 98 & & & 33 & \\
\hline decyl myristate & $\mathrm{C} 24 \mathrm{H} 48 \mathrm{O} 2$ & $41297-71-3$ & & & & & 72 & & 32 & [21],[26] \\
\hline dodecyl laurate & $\mathrm{C} 24 \mathrm{H} 48 \mathrm{O} 2$ & $13945-76-1$ & & & & & 85 & & 32 & [21],[26] \\
\hline 2-ethylhexyl stearate & $\mathrm{C} 26 \mathrm{H} 52 \mathrm{O} 2$ & $22047-49-0$ & & & & 116 & & & 33 & \\
\hline decyl palmitate & $\mathrm{C} 26 \mathrm{H} 52 \mathrm{O} 2$ & $42232-27-9$ & & & & & 91 & & 32 & {$[21],[26]$} \\
\hline dodecyl myristate & $\mathrm{C} 26 \mathrm{H} 52 \mathrm{O} 2$ & $2040-64-4$ & & & & & 74 & & 32 & {$[21],[26]$} \\
\hline hexadecyl laurate & $\mathrm{C} 28 \mathrm{H} 56 \mathrm{O} 2$ & $8038-55-9$ & & & & & 88 & & 32 & {$[21],[26]$} \\
\hline
\end{tabular}


A 7.2 Esters: Unsaturated

\begin{tabular}{|c|c|c|c|c|c|c|c|c|c|c|}
\hline Compound & Formula & CAS & $\begin{array}{l}\text { ASTM } \\
\text { Method } \\
\text { D613 } \\
\text { (CFR) }\end{array}$ & $\begin{array}{c}\text { ASTM } \\
\text { Method } \\
\text { D6890 } \\
\text { (IQT) }\end{array}$ & $\begin{array}{l}\text { ASTM } \\
\text { Method } \\
\text { D7170 } \\
\text { (FIT) }\end{array}$ & $\begin{array}{l}\text { Other } \\
\text { Ignition } \\
\text { Delay } \\
\text { Method }\end{array}$ & Blend & $\begin{array}{l}\text { Unknown } \\
\text { Method }\end{array}$ & Ref. & Notes \\
\hline methyl sorbate & $\mathrm{C} 7 \mathrm{H} 10 \mathrm{O} 2$ & $689-89-4$ & & 6.0 & & & & & 41 & \\
\hline methyl-9-decenoate & $\mathrm{C} 11 \mathrm{H} 20 \mathrm{O} 2$ & & & 38.3 & & & & & 41 & [7] \\
\hline vinyl laurate & $\mathrm{C} 14 \mathrm{H} 26 \mathrm{O} 2$ & $2146-71-6$ & & 77.0 & & & & & 76 & new \\
\hline \multirow[t]{2}{*}{ methyl palmitoleate } & \multirow[t]{2}{*}{$\mathrm{C} 17 \mathrm{H} 32 \mathrm{O} 2$} & \multirow[t]{2}{*}{$1120-25-8$} & & & & 51 & & & 33 & \\
\hline & & & & 56.6 & & & & & 68 & new \\
\hline \multirow[t]{3}{*}{ methyl linolenate } & \multirow[t]{3}{*}{$\mathrm{C} 19 \mathrm{H} 32 \mathrm{O} 2$} & \multirow[t]{3}{*}{$301-00-8$} & 45.9 & & & & & & 12 & [36] \\
\hline & & & & & & 23 & & & 34 & \\
\hline & & & & 37.0 & & & & & 41 & [2] \\
\hline methyl $\alpha$-linolenate & $\mathrm{C} 19 \mathrm{H} 32 \mathrm{O} 2$ & & & 22.7 & & & & & 46 & \\
\hline methyl $\nu$-linolenate & $\mathrm{C} 19 \mathrm{H} 32 \mathrm{O} 2$ & & & 29.2 & & & & & 45 & \\
\hline \multirow[t]{6}{*}{ methyl linoleate } & \multirow[t]{6}{*}{$\mathrm{C} 19 \mathrm{H} 34 \mathrm{O} 2$} & \multirow[t]{6}{*}{$112-63-0$} & 41.7 & & & & & & 12 & [35] \\
\hline & & & & 43.9 & & & & & 41 & [2] \\
\hline & & & & 38.2 & & & & & 42 & \\
\hline & & & & & & 38 & & & 33 & \\
\hline & & & & & & 42 & & & 34 & \\
\hline & & & & & & & & 43 & 22 & \\
\hline methyl linolelaidate & $\mathrm{C} 19 \mathrm{H} 34 \mathrm{O} 2$ & $2566-97-4$ & & 43.0 & & & & & 46 & \\
\hline methyl elaidate & $\mathrm{C} 19 \mathrm{H} 36 \mathrm{O} 2$ & $1937-62-8$ & & 57.2 & & & & & 46 & \\
\hline
\end{tabular}




\begin{tabular}{|c|c|c|c|c|c|c|c|c|c|c|}
\hline Compound & Formula & CAS & $\begin{array}{l}\text { ASTM } \\
\text { Method } \\
\text { D613 } \\
\text { (CFR) }\end{array}$ & $\begin{array}{l}\text { ASTM } \\
\text { Method } \\
\text { D6890 } \\
\text { (IQT) }\end{array}$ & $\begin{array}{l}\text { ASTM } \\
\text { Method } \\
\text { D7170 } \\
\text { (FIT) }\end{array}$ & $\begin{array}{l}\text { Other } \\
\text { Ignition } \\
\text { Delay } \\
\text { Method }\end{array}$ & Blend & $\begin{array}{l}\text { Unknown } \\
\text { Method }\end{array}$ & Ref. & Notes \\
\hline \multirow[t]{10}{*}{ methyl oleate } & \multirow[t]{10}{*}{$\mathrm{C} 19 \mathrm{H} 36 \mathrm{O} 2$} & \multirow[t]{10}{*}{$112-62-9$} & 56.0 & & & & & & 12 & [35] \\
\hline & & & & 59.8 & & & & & 41 & [2], [20] \\
\hline & & & & 56.6 & & & & & 42 & \\
\hline & & & & 59.3 & & & & & 42 & \\
\hline & & & & & & 80 & & & 19 & \\
\hline & & & & & & 59 & & & 33 & \\
\hline & & & & & & 55 & & & 34 & \\
\hline & & & & & & & 71 & & 32 & [21], [26] \\
\hline & & & & & & & & 53 & 22 & \\
\hline & & & & 56.6 & & & & & 68 & new \\
\hline \multirow[t]{2}{*}{ methyl ricinoleate } & \multirow[t]{2}{*}{$\mathrm{C} 19 \mathrm{H} 36 \mathrm{O} 3$} & \multirow[t]{2}{*}{$141-24-2$} & & 37.4 & & & & & 68 & new \\
\hline & & & & 37.4 & & & & & 42 & \\
\hline methyl asclepate & $\mathrm{C} 19 \mathrm{H} 3802$ & & & 53.9 & & & & & 46 & \\
\hline ethyl linolenate & $\mathrm{C} 2 \mathrm{OH} 34 \mathrm{O} 2$ & $1191-41-9$ & & & & 27 & & & 34 & \\
\hline \multirow[t]{3}{*}{ ethyl linoleate } & \multirow[t]{3}{*}{$\mathrm{C} 2 \mathrm{OH} 36 \mathrm{O} 2$} & \multirow[t]{3}{*}{$544-35-4$} & 44.4 & & & & & & 12 & [35] \\
\hline & & & & & & 40 & & & 33 & \\
\hline & & & & & & 37 & & & 34 & \\
\hline \multirow[t]{3}{*}{ ethyl oleate } & \multirow[t]{3}{*}{$\mathrm{C} 2 \mathrm{OH} 38 \mathrm{O} 2$} & \multirow[t]{3}{*}{$111-62-6$} & & & & 68 & & & 33 & \\
\hline & & & & & & 54 & & & 34 & \\
\hline & & & & & & & 72 & & 32 & [21], [26] \\
\hline $\begin{array}{c}\text { methyl- } \\
5(Z) 8(z) 11(Z) 14(Z)- \\
\text { eicosatetraenoate }\end{array}$ & $\mathrm{C} 21 \mathrm{H} 34 \mathrm{O} 2$ & & & 29.6 & & & & & 44 & \\
\hline
\end{tabular}




\begin{tabular}{|c|c|c|c|c|c|c|c|c|c|c|}
\hline Compound & Formula & CAS & $\begin{array}{l}\text { ASTM } \\
\text { Method } \\
\text { D613 } \\
\text { (CFR) }\end{array}$ & $\begin{array}{l}\text { ASTM } \\
\text { Method } \\
\text { D6890 } \\
\text { (IQT) }\end{array}$ & $\begin{array}{l}\text { ASTM } \\
\text { Method } \\
\text { D7170 } \\
\text { (FIT) }\end{array}$ & $\begin{array}{l}\text { Other } \\
\text { Ignition } \\
\text { Delay } \\
\text { Method }\end{array}$ & Blend & $\begin{array}{l}\text { Unknown } \\
\text { Method }\end{array}$ & Ref. & Notes \\
\hline \multirow[t]{2}{*}{ propyl linoleate } & \multirow[t]{2}{*}{$\mathrm{C} 21 \mathrm{H} 38 \mathrm{O} 2$} & \multirow[t]{2}{*}{$38433-95-3$} & & & & 44 & & & 33 & \\
\hline & & & & & & 41 & & & 34 & \\
\hline isopropyl oleate & $\mathrm{C} 21 \mathrm{H} 40 \mathrm{O} 2$ & $112-11-8$ & & & & 87 & & & 33 & \\
\hline \multirow[t]{3}{*}{ propyl oleate } & \multirow[t]{3}{*}{$\mathrm{C} 21 \mathrm{H} 40 \mathrm{O} 2$} & \multirow[t]{3}{*}{$111-59-1$} & & & & & 72 & & 32 & [21], [26] \\
\hline & & & & & & 59 & & & 33 & \\
\hline & & & & & & 56 & & & 34 & \\
\hline \multirow[t]{2}{*}{ butyl linolenate } & \multirow[t]{2}{*}{$\mathrm{C} 22 \mathrm{H} 38 \mathrm{O} 2$} & & & & & 29 & & & 34 & \\
\hline & & & & & & 42 & & & 34 & \\
\hline butyl linoleate & $\mathrm{C} 22 \mathrm{H} 40 \mathrm{O} 2$ & & & & & 54 & & & 33 & \\
\hline \multirow[t]{3}{*}{ butyl oleate } & \multirow[t]{3}{*}{$\mathrm{C} 22 \mathrm{H} 42 \mathrm{O} 2$} & \multirow[t]{3}{*}{$142-77-8$} & & & & 60 & & & 34 & \\
\hline & & & & & & 62 & & & 33,35 & \\
\hline & & & & & & & 102 & & 32 & [21], [26] \\
\hline isobutyl oleate & $\mathrm{C} 22 \mathrm{H} 42 \mathrm{O} 2$ & $10024-47-2$ & & & & 60 & & & 33 & \\
\hline $\begin{array}{c}\text { methyl } \\
4(Z), 7(Z), 10(Z), 13(Z) \\
16(Z), 19(Z)- \\
\text { docosahexaenoate }\end{array}$ & $\mathrm{C} 23 \mathrm{H} 34 \mathrm{O} 2$ & $28061-46-3$ & & 24.4 & & & & & 44 & \\
\hline methyl erucate & $\mathrm{C} 23 \mathrm{H} 44 \mathrm{O} 2$ & 1120-34-9 & & 74.2 & & & & & & \\
\hline hexyl oleate & $\mathrm{C} 24 \mathrm{H} 46 \mathrm{O} 2$ & $20290-84-0$ & & & & & 102 & & & \\
\hline 2-ethylhexyl oleate & $\mathrm{C} 26 \mathrm{H} 50 \mathrm{O} 2$ & $26399-02-0$ & & & & 88 & & & 33 & \\
\hline octyl oleate & $\mathrm{C} 26 \mathrm{H} 50 \mathrm{O} 2$ & $32953-65-4$ & & & & & 131 & & 32 & {$[21],[26]$} \\
\hline
\end{tabular}




\section{A.7.3 Esters: Diesters/Triglycerides}

\begin{tabular}{|c|c|c|c|c|c|c|c|c|c|c|}
\hline Compound & Formula & CAS & $\begin{array}{l}\text { ASTM } \\
\text { Method } \\
\text { D613 } \\
\text { (CFR) }\end{array}$ & $\begin{array}{l}\text { ASTM } \\
\text { Method } \\
\text { D6890 } \\
\text { (IQT) }\end{array}$ & $\begin{array}{c}\text { ASTM } \\
\text { Method } \\
\text { D7170 } \\
\text { (FIT) }\end{array}$ & $\begin{array}{l}\text { Other } \\
\text { Ignition } \\
\text { Delay } \\
\text { Method }\end{array}$ & Blend & $\begin{array}{l}\text { Unknown } \\
\text { Method }\end{array}$ & Ref. & Notes \\
\hline dimethyl malonate & $\mathrm{C} 5 \mathrm{H} 8 \mathrm{O} 4$ & $108-59-8$ & & & & & 15 & & 32 & $\begin{array}{c}\text { [21],[26], } \\
{[37]}\end{array}$ \\
\hline diethyl oxalate & $\mathrm{C} 6 \mathrm{H} 10 \mathrm{O} 4$ & $95-92-1$ & & & & & 21 & & 32 & $\begin{array}{c}\text { [21],[26], } \\
{[37]}\end{array}$ \\
\hline diethyl malonate & $\mathrm{C} 7 \mathrm{H} 12 \mathrm{O} 4$ & $105-53-3$ & & & & & 15 & & 32 & $\begin{array}{c}\text { [21],[26], } \\
{[37]}\end{array}$ \\
\hline diethyl butanedioate & $\mathrm{C} 8 \mathrm{H} 14 \mathrm{O} 4$ & $123-25-1$ & 21.0 & & & & & & 52 & [18] \\
\hline diethyl succinate & $\mathrm{C} 8 \mathrm{H} 14 \mathrm{O} 4$ & $627-93-0$ & & & & & 14 & & 32 & $\begin{array}{c}\text { [21],[26], } \\
{[37]}\end{array}$ \\
\hline dimethyl adipate & $\mathrm{C} 8 \mathrm{H} 14 \mathrm{O} 4$ & $105-99-7$ & & & & & 5 & & 32 & $\begin{array}{c}\text { [21],[26], } \\
{[37]}\end{array}$ \\
\hline glycerol triacetate & $\mathrm{C} 9 \mathrm{H} 14 \mathrm{O} 6$ & $102-76-1$ & & $<5$ & & & & & 41 & [17] \\
\hline dimethyl phthalate & $\mathrm{C} 10 \mathrm{H} 10 \mathrm{O} 4$ & $131-11-3$ & & & & & 19 & & 32 & $\begin{array}{c}\text { [21],[26], } \\
{[37]}\end{array}$ \\
\hline diethyl adipate & $\mathrm{C} 10 \mathrm{H} 18 \mathrm{O} 4$ & $141-28-6$ & & & & & 15 & & 24 & [24] \\
\hline dibutyl malonate & $\mathrm{C} 11 \mathrm{H} 20 \mathrm{O} 4$ & $1190-39-2$ & 21.0 & & & & & & 52 & [18] \\
\hline dimethyl azelate & $\mathrm{C} 11 \mathrm{H} 20 \mathrm{O} 4$ & $1732-10-1$ & & & & & 24 & & 32 & $\begin{array}{c}\text { [21],[26], } \\
{[37]}\end{array}$ \\
\hline dibutyl butanedioate & $\mathrm{C} 12 \mathrm{H} 14 \mathrm{O} 4$ & $141-03-7$ & 21.0 & & & & & & 52 & [18] \\
\hline dibutyl butanedioate & $\mathrm{C} 12 \mathrm{H} 14 \mathrm{O} 4$ & $141-03-7$ & 21.0 & & & & & & 52 & [18] \\
\hline dibutyl fumarate & $\mathrm{C} 12 \mathrm{H} 20 \mathrm{O} 4$ & $105-75-9$ & 23.0 & & & & & & 52 & [18] \\
\hline
\end{tabular}




\begin{tabular}{|c|c|c|c|c|c|c|c|c|c|c|}
\hline Compound & Formula & CAS & $\begin{array}{l}\text { ASTM } \\
\text { Method } \\
\text { D613 } \\
\text { (CFR) }\end{array}$ & $\begin{array}{c}\text { ASTM } \\
\text { Method } \\
\text { D6890 } \\
\text { (IQT) }\end{array}$ & $\begin{array}{l}\text { ASTM } \\
\text { Method } \\
\text { D7170 } \\
\text { (FIT) }\end{array}$ & $\begin{array}{l}\text { Other } \\
\text { Ignition } \\
\text { Delay } \\
\text { Method }\end{array}$ & Blend & $\begin{array}{l}\text { Unknown } \\
\text { Method }\end{array}$ & Ref. & Notes \\
\hline \multirow[t]{2}{*}{ dibutyl maleate } & \multirow[t]{2}{*}{$\mathrm{C} 12 \mathrm{H} 20 \mathrm{O} 4$} & \multirow[t]{2}{*}{$105-76-0$} & & & & & 29 & & 24 & [24] \\
\hline & & & & & & & & 28 & 47 & \\
\hline diethyl azelate & $\mathrm{C} 13 \mathrm{H} 24 \mathrm{O} 4$ & $624-17-9$ & & & & & 47 & & 32 & $\begin{array}{c}\text { [21],[26], } \\
\text { [37] }\end{array}$ \\
\hline diethyl sebacate & $\mathrm{C} 14 \mathrm{H} 26 \mathrm{O} 4$ & $110-40-7$ & & & & & 47 & & 32 & $\begin{array}{c}{[21],[26],} \\
{[37]}\end{array}$ \\
\hline dibutyl adipate & $\mathrm{C} 14 \mathrm{H} 26 \mathrm{O} 4$ & $105-99-7$ & & & & & 81 & & 32 & $\begin{array}{c}\text { [21],[26], } \\
{[37]}\end{array}$ \\
\hline tributyrin & $\mathrm{C} 15 \mathrm{H} 26 \mathrm{O} 6$ & $60-01-5$ & & & & & -5 & & 24 & [24] \\
\hline dibutyl phthalate & $\mathrm{C} 16 \mathrm{H} 22 \mathrm{O} 4$ & $87-74-2$ & & & & & 38 & & 32 & $\begin{array}{c}\text { [21],[26], } \\
{[37]}\end{array}$ \\
\hline dibutyl azelate & $\mathrm{C} 17 \mathrm{H} 32 \mathrm{O} 4$ & 2917-13-9 & & & & & 83 & & 32 & $\begin{array}{c}{[21],[26],} \\
{[37]}\end{array}$ \\
\hline dihexyl phthalate & $\mathrm{C} 2 \mathrm{OH} 30 \mathrm{O} 4$ & $84-75-3$ & & & & & 48 & & 32 & $\begin{array}{c}\text { [21],[26], } \\
\text { [37] }\end{array}$ \\
\hline dibutyl succinate & $\mathrm{C} 12 \mathrm{H} 22 \mathrm{O} 4$ & $141-03-7$ & & & 13.0 & & & & 67 & \\
\hline dihexyl azelate & $\mathrm{C} 21 \mathrm{H} 40 \mathrm{O} 4$ & $109-31-9$ & & & & & 99 & & 32 & $\begin{array}{c}\text { [21],[26], } \\
{[37]}\end{array}$ \\
\hline dioctyl adipate & $\mathrm{C} 22 \mathrm{H} 42 \mathrm{O} 4$ & $123-79-5$ & & & & & 89 & & 32 & $\begin{array}{c}{[21],[26],} \\
{[37]}\end{array}$ \\
\hline dioctyl sebacate & $\mathrm{C} 26 \mathrm{H} 50 \mathrm{O} 4$ & $122-62-3$ & & & & & 70 & & 32 & $\begin{array}{c}\text { [21],[26], } \\
{[37]}\end{array}$ \\
\hline trilaurin & $\mathrm{C} 39 \mathrm{H} 74 \mathrm{O} 6$ & $538-24-9$ & & & & 100 & & & 19 & \\
\hline trimyristin & $\mathrm{C} 45 \mathrm{H} 86 \mathrm{O} 6$ & $555-45-3$ & & & & 100 & & & 19 & \\
\hline
\end{tabular}




\begin{tabular}{|c|c|c|c|c|c|c|c|c|c|c|}
\hline Compound & Formula & CAS & $\begin{array}{l}\text { ASTM } \\
\text { Method } \\
\text { D613 } \\
\text { (CFR) }\end{array}$ & $\begin{array}{c}\text { ASTM } \\
\text { Method } \\
\text { D6890 } \\
\text { (IQT) }\end{array}$ & $\begin{array}{c}\text { ASTM } \\
\text { Method } \\
\text { D7170 } \\
\text { (FIT) }\end{array}$ & $\begin{array}{l}\text { Other } \\
\text { Ignition } \\
\text { Delay } \\
\text { Method }\end{array}$ & Blend & $\begin{array}{l}\text { Unknown } \\
\text { Method }\end{array}$ & Ref. & Notes \\
\hline tripalmitin & С51H98O6 & $555-44-2$ & & & & 89 & & & 19 & \\
\hline triolein & C57H104O6 & $122-32-7$ & & & & 45 & & & 19 & \\
\hline tristearin & C57H110O6 & $555-43-1$ & & & & 85 & & & 19 & \\
\hline trilinolenin & $\mathrm{C} 57 \mathrm{H} 92 \mathrm{O} 6$ & $14465-68-0$ & & & & 23 & & & 19 & \\
\hline trilinolein & C57H98O6 & $537-40-6$ & & & & 32 & & & 19 & \\
\hline
\end{tabular}


A. 8 Acids

\begin{tabular}{|c|c|c|c|c|c|c|c|c|c|c|}
\hline Compound & Formula & CAS & $\begin{array}{l}\text { ASTM } \\
\text { Method } \\
\text { D613 } \\
\text { (CFR) }\end{array}$ & $\begin{array}{c}\text { ASTM } \\
\text { Method } \\
\text { D6890 } \\
\text { (IQT) }\end{array}$ & $\begin{array}{l}\text { ASTM } \\
\text { Method } \\
\text { D7170 } \\
\text { (FIT) }\end{array}$ & $\begin{array}{l}\text { Other } \\
\text { Ignition } \\
\text { Delay } \\
\text { Method }\end{array}$ & Blend & $\begin{array}{l}\text { Unknown } \\
\text { Method }\end{array}$ & Ref. & Notes \\
\hline decanoic acid & $\mathrm{C} 10 \mathrm{H} 20 \mathrm{O} 2$ & $334-48-5$ & & & & & 48 & & 31 & $\begin{array}{l}{[29],} \\
{[31]}\end{array}$ \\
\hline linolenic acid & $\mathrm{C} 18 \mathrm{H} 30 \mathrm{O} 2$ & $463-40-1$ & & & & 20 & & & 34 & \\
\hline linoleic acid & $\mathrm{C} 18 \mathrm{H} 32 \mathrm{O} 2$ & $60-33-3$ & & & & 31 & & & 34 & \\
\hline oleic acid & $\mathrm{C} 18 \mathrm{H} 34 \mathrm{O} 2$ & $112-80-1$ & & & & 46 & & & 34 & \\
\hline stearic acid & $\mathrm{C} 18 \mathrm{H} 36 \mathrm{O} 2$ & $57-11-4$ & & & & 62 & & & 34 & [5] \\
\hline
\end{tabular}




\section{A.9 Furans}

\begin{tabular}{|c|c|c|c|c|c|c|c|c|c|c|}
\hline Compound & Formula & CAS & $\begin{array}{l}\text { ASTM } \\
\text { Method } \\
\text { D613 } \\
\text { (CFR) }\end{array}$ & $\begin{array}{c}\text { ASTM } \\
\text { Method } \\
\text { D6890 } \\
\text { (IQT) }\end{array}$ & $\begin{array}{l}\text { ASTM } \\
\text { Method } \\
\text { D7170 } \\
\text { (FIT) }\end{array}$ & $\begin{array}{l}\text { Other } \\
\text { Ignition } \\
\text { Delay } \\
\text { Method }\end{array}$ & Blend & $\begin{array}{l}\text { Unknown } \\
\text { Method }\end{array}$ & Ref. & Notes \\
\hline furan & $\mathrm{C} 4 \mathrm{H} 4 \mathrm{O}$ & $110-00-9$ & & 7.0 & & & & & 72 & new \\
\hline furfural & $\mathrm{C} 5 \mathrm{H} 4 \mathrm{O} 2$ & $98-01-1$ & & 13.9 & & & & & 76 & new \\
\hline \multirow[t]{2}{*}{ 2-methyl furan } & \multirow[t]{2}{*}{$\mathrm{C} 5 \mathrm{H} 6 \mathrm{O}$} & \multirow[t]{2}{*}{$534-22-5$} & & 8.9 & & & & & 72 & new \\
\hline & & & & 9.1 & & & & & 76 & new \\
\hline \multirow[t]{2}{*}{ 2-furfuryl alcohol } & \multirow[t]{2}{*}{$\mathrm{C} 5 \mathrm{H} 6 \mathrm{O} 2$} & \multirow[t]{2}{*}{$98-00-0$} & & 10.8 & & & & & 72 & new \\
\hline & & & & 9.7 & & & & & 76 & new \\
\hline 2,5 dimethyl furan & $\mathrm{C} 6 \mathrm{H} 8 \mathrm{O}$ & $625-86-5$ & & 10.9 & & & & & 72 & new \\
\hline 2-ethyl furan & $\mathrm{C} 6 \mathrm{H} 8 \mathrm{O}$ & $3208-16-0$ & & 10.2 & & & & & 72 & new \\
\hline 2-butyl furan & $\mathrm{C} 8 \mathrm{H} 12 \mathrm{O}$ & $4466-24-4$ & & 13.1 & & & & & 72 & new \\
\hline furfuryl ethyl ether & $\mathrm{C} 7 \mathrm{H} 10 \mathrm{O} 2$ & $6270-56-0$ & & 18.4 & & & & & 41 & new \\
\hline
\end{tabular}




\section{Notes}

[1] $20 \%$ blend.

[2] All new data updated to use D6890-06 DCN correlation: $\mathrm{DCN}=4.460+186.6 /$ ignition delay (except when ignition delay is outside range of 3.3 to 6.4 milliseconds, then use original equation) including data collected before spec came into effect.

[3] Data already in Compendium, also updated with new correlation between ignition delay for IQT in ASTM D6890-06 and later versions.

[4] Primary reference fuel, value set by ASTM D6890 for IQT.

[5] Compound solid at room temperature, must be heated to test.

[6] Blend tested on IQT.

[7] Compound was treated with silica gel to remove contaminants.

[8] Calculated from cetene value $\times 0.875$.

[9] Primary reference fuel value set by ASTM D613 for CFR.

[10] cis-Isomer surmised from boiling point and density data.

[11] Original reference says this is an "extrapolated value."

[12] Estimated DCN is $\mathrm{Y}$-intercept from regression of compound blends with $\mathrm{n}$-heptane.

[13] Sample contained oxidation inhibitor.

[14] Original source states: "These compounds are apparently two isomers on n-butyltetralin," but does not specify which is which.

[15] Because of small difference in blend $\mathrm{CN}$ and base fuel $\mathrm{CN}$, blend calculation is not very meaningful.

[16] Delay values are from correlations with reference fuels and with diesel fuels, respectively.

[17] Actual value may be much less than 5 .

[18] ISO 5165, European equivalent to ASTM D613.

[19] Value is from double blend procedure with potential for large errors.

[20] Fuel reservoir and line heated to $55^{\circ} \mathrm{C}$.

[21] Used BASF engine and DIN 51773.

[22] Reference says cetane number collected by "engine method."

[23] Cetane number changed from 2004 Compendium.

[24] Based on approx. 20\% blend.

[25] Based on a $30 \%$ blend. 
[26] Based on a 5\%-7\% blend.

[27] Measured using ASTM D613-43T (a temporary version of standard).

[28] Based on $80 \%$ blend.

[29] Based on $70 \%$ blend.

[30] Measured using ASTM D613-59T (a temporary version of the standard).

[31] Blend tested using ASTM D613.

[32] From 14 ternary mixtures of n-decane, iso-octane, and toluene.

[33] From 43 mixtures of n-dodecane/iso-octane/1,3,5-trimethylbenzene/n-propylbenzene.

[34] Optimized from [32] and another set of 15 blends.

[35] Test method changed from 2004 Compendium.

[36] Name of compound changed from 2004 Compendium.

[37] Measurement not included in 2004 Compendium, although reference had been included.

[38] Dipropylene glycol methyl ether, mixed isomers, aka DOWANOL DPM glycol ether. 


\section{Appendix B. Sources for Cetane Number Data}

\begin{tabular}{|c|c|}
\hline Number & Reference \\
\hline 1 & $\begin{array}{l}\text { Olson, D.R.; Meckel, N.T.; Quillian, R.D. (1960). "Combustion Characteristics of } \\
\text { Compression Ignition Engine Fuel Components." SAE Technical Paper } 600112 . \\
\text { http://dx.doi.org/10.4271/600112 }\end{array}$ \\
\hline 2 & $\begin{array}{l}\text { Hardenberg, H.O. (1984). "Zundwilligkeit und Cetanzahl reiner Kohlenwasserstoffe." } \\
\text { Mineraloeltechnik (29); p. } 13 .\end{array}$ \\
\hline 3 & $\begin{array}{l}\text { Puckett, A.D.; Caudle, B.H. (1948). Ignition Qualities of Hydrocarbons in the Diesel Fuel } \\
\text { Boiling Range. Washington, DC: U.S. Department of the Interior, Bureau of Mines } \\
\text { Information Circular 7474. }\end{array}$ \\
\hline 4 & $\begin{array}{l}\text { Rose, J.W.; Cooper, J.R., eds. (1977). "Detonation of Liquid Fuels." In Technical Data on } \\
\text { Fuel, British National Committee, World Energy Conference, p. } 285 .\end{array}$ \\
\hline 5 & $\begin{array}{l}\text { Hurn, R.W.; Smith, H.M. (1951). "Hydrocarbons in the Diesel Boiling Range." Industrial and } \\
\text { Engineering Chemistry (43:12); pp. 2788-2793. http://dx.doi.org/10.1021/ie50504a044 }\end{array}$ \\
\hline 6 & $\begin{array}{l}\text { Gulder, O.L.; Glavincevski, B.; Kallio, N.N. (1989). "A Rapid Cetane Number Prediction } \\
\text { Method for Petroleum Liquids and Pure Hydrocarbons Using Proton NMR." SAE Technical } \\
\text { Paper 892073. http://dx.doi.org/10.4271/892073 }\end{array}$ \\
\hline 7 & $\begin{array}{l}\text { Chevron. (2007). Diesel Fuels Technical Review FTR- } \\
\text { 2. https://www.google.com/url?q=http://www.chevronwithtechron.ca/products/documents/D } \\
\text { iesel Fuel Tech Review.pdf\&sa=U\&ei=eFiGU6GSIcubyATEm4DoDQ\&ved=0CCIQFjABO } \\
\text { Ao\&usg=AFQjCNEbGC9eSkLXIUSKcYGid5NaGIPK-A }\end{array}$ \\
\hline 8 & $\begin{array}{l}\text { American Society for Testing and Materials. (1958). Knocking Characteristics of Pure } \\
\text { Hydrocarbons. ASTM Special Technical Publication 225, developed under American } \\
\text { Petroleum Institute Research Project } 45 .\end{array}$ \\
\hline 9 & $\begin{array}{l}\text { Ryan, T.W.; Stapper, B. (1987). "Diesel Fuel Ignition Quality as Determined in a Constant } \\
\text { Volume Combustion Bomb." SAE Technical Paper } \\
\text { 870586. http://dx.doi.org/10.4271/870586 }\end{array}$ \\
\hline 10 & Petrov, A.D. (1946). "Cetene Number Data." Bull. Acad. Sci. USSR (4) p. 543. \\
\hline 11 & Guibet, J.-C. (1999). Fuels and Engines. (1); p. 339. \\
\hline 12 & $\begin{array}{l}\text { McCormick, R.L.; Grabowski, M.S.; Alleman, T.L.; Herring, A.M.; Tyson, K.S. (2001). } \\
\text { "Impact of Biodiesel Source Material and Chemical Structure on Emissions of Criteria } \\
\text { Pollutants from a Heavy-Duty Engine." Environ. Sci. Tech. (35:9) p. 1742- } \\
\text { 1747. http://dx.doi.org/10.1021/es001636t }\end{array}$ \\
\hline 13 & $\begin{array}{l}\text { Kroeger, C.A. (1986). "A Neat Methanol Direct Injection Combustion System for Heavy- } \\
\text { Duty Applications." SAE Technical Paper 861169. http://dx.doi.org/10.4271/861169 }\end{array}$ \\
\hline 14 & $\begin{array}{l}\text { McCormick, R.L. (2002). "Technical Barriers to the Use of Ethanol in Diesel Fuel." 7th } \\
\text { Annual National Ethanol Conference, } 27 \text { Feb-1 Mar } 2002 .\end{array}$ \\
\hline 15 & $\begin{array}{l}\text { Tijm, P.J.A. (1998). "Overview of Cetaner for Diesel Fuel and an AET Study of Cetaner } \\
\text { Blended with Low Cetane Diesel Fuel." Windsor Workshop, 8-10 June } 1998 .\end{array}$ \\
\hline 16 & $\begin{array}{l}\text { Hardenberg, H.O.; Schaefer, A.J. (1981). "The Use of Ethanol as a Fuel for Compression } \\
\text { Ignition Engines." SAE Technical Paper 811211. http://dx.doi.org/10.4271/811211 }\end{array}$ \\
\hline
\end{tabular}




\begin{tabular}{|c|c|}
\hline Number & Reference \\
\hline 17 & $\begin{array}{l}\text { Hardenberg, H.O.; Ehnert, E.R. "Ignition Quality Determination Problems with Alternative } \\
\text { Fuels for Compression Ignition Engines." SAE Technical Paper } \\
\text { 811212. http://dx.doi.org/10.4271/811212 }\end{array}$ \\
\hline 18 & $\begin{array}{l}\text { Freedman, B.; Bagby, M.O. (1990). "Predicting Cetane Numbers of n-Alcohols and Methyl } \\
\text { Esters from their Physical Properties." J. American Oil Chemists Society (67:9) pp. 565- } \\
\text { 571. http://dx.doi.org/10.1007/BF02540768 }\end{array}$ \\
\hline 19 & $\begin{array}{l}\text { Freedman, B.; Bagby, M.; Callahan, T.; Ryan, T. (1990). "Cetane Numbers of Fatty Esters, } \\
\text { Fatty Alcohols and Triglycerides Determined in a Constant Volume Combustion Bomb." } \\
\text { SAE Technical Paper 900343. http://dx.doi.org/10.4271/900343 }\end{array}$ \\
\hline 20 & $\begin{array}{l}\text { Fleisch, T.; McCarthy, C.; Basu, A.; Udovich, C.; Charbonneau, P.; Slodowske, W.; } \\
\text { Mikkelsen, S.-E.; McCandless, J. (1995). "A New Clean Diesel Technology: Demonstration } \\
\text { of ULEV Emissions on a Navistar Diesel Engine Fueled with Dimethyl Ether." SAE } \\
\text { Technical Paper 950061. http://dx.doi.org/10.4271/950061 }\end{array}$ \\
\hline 21 & $\begin{array}{l}\text { Moulton, D.F.; Naegeli, D.W. (1998). “Oxygenates for Diesel Fuel.” Windsor Workshop } \\
\text { paper, } 9 \text { June } 1998 .\end{array}$ \\
\hline 22 & $\begin{array}{l}\text { Shay, E.G. (1993). "Diesel Fuel from Vegetable Oils: Status and Opportunities." Biomass } \\
\text { and Bioenergy (4:4); pp. 227-242. http://dx.doi.org/10.1016/0961-9534(93)90080-N }\end{array}$ \\
\hline 23 & $\begin{array}{l}\text { Vertin, K.; Ohi, J.; Naegeli, D.; Childress, K.; Hagen, G.P.; McCarthy, C.I.; Cheng, A.S.; } \\
\text { Dibble, R.W. (1999). "Methylal and Methylal-Diesel Blended Fuels for Use in Compression- } \\
\text { Ignition Engines." SAE Technical Paper 1999-01-1508. http://dx.doi.org/10.4271/1999-01- } \\
\underline{1508}\end{array}$ \\
\hline 24 & $\begin{array}{l}\text { Natarajan, M.; Frame, E.A.; Naegeli, D.W.; Asmus, T.; Clark, W.; Garbak, J.; Manue, A.; } \\
\text { Gonzales, D.; Liney, E.; Piel, W.; Wallace III, J.P. (2001). "Oxygenates for Advanced } \\
\text { Petroleum-Based Diesel Fuels: Part 1. Screening and Selection Methodology for the } \\
\text { Oxygenates." SAE Technical Paper 2001-01-3631. http://dx.doi.org/10.4271/2001-01-3631 }\end{array}$ \\
\hline 25 & $\begin{array}{l}\text { Lambiotte et Cie. (1998). The Use of Lambiotte Acetals in Diesel Fuels. Lambiotte report, } \\
28 \text { May. }\end{array}$ \\
\hline 26 & $\begin{array}{l}\text { Beatrice, C.; Bertoli, C.; D'Alessio, J.; del Giocomo, N.; Lazzaro, M.; Massoli, P. (1996). } \\
\text { "Experimental Characterization of Combustion Behavior of New Diesel Fuels for Low } \\
\text { Emission Engines." Combustion Science and Technology (120) pp. 335- } \\
\text { 355. http://dx.doi.org/10.1080/00102209608935580 }\end{array}$ \\
\hline 27 & $\begin{array}{l}\text { Miyamoto, N.; Ogawa, H.; Nurun, N.; Obata, K.; Arima, T. (1998). "Smokeless, Low NOx, } \\
\text { High Thermal Efficiency, and Low Noise Diesel Combustion with Oxygenated Agents as } \\
\text { Main Fuel." SAE Technical Paper 980506. DOI: } 10.4271 / 980506\end{array}$ \\
\hline 28 & $\begin{array}{l}\text { Zhu, J.; Cao, X.-L.; Pigeon, R.; Mitchell, K. (2003).”Comparison of Vehicle Exhaust } \\
\text { Emissions from Modified Diesel Fuels," J. Air Waste Manag. Assoc. (53:1); p. 67- } \\
\text { 76. http://dx.doi.org/10.1080/10473289.2003.10466125 }\end{array}$ \\
\hline 29 & $\begin{array}{l}\text { Pigeon, R. (Jan. 14, 2002). "Pure and Blending Properties of Two Di-ether Diesel Blends." } \\
\text { Personal communication. }\end{array}$ \\
\hline 30 & Deleted. \\
\hline 31 & $\begin{array}{l}\text { Klopfenstein, W.E. (1985). "Effect of Molecular Weights of Fatty Acid Esters on Cetane } \\
\text { Numbers as Diesel Fuels." J. Am. Oil Chemists Soc. (62:6); pp. 1029-1031. } \\
\text { DOI: http://dx.doi.org/10.1007/BF02935708 }\end{array}$ \\
\hline
\end{tabular}




\begin{tabular}{|c|c|}
\hline Number & Reference \\
\hline 32 & $\begin{array}{l}\text { Serdari, A.; Lois, E.; Stournas, S. (1999). "Impact of Esters of Mono- and Dicarboxylic } \\
\text { Acids on Diesel Fuel Quality." Ind. Eng. Chem. Res. (38:9) pp. 3543-3548. } \\
\text { http://dx.doi.org/10.1021/ie9900115 }\end{array}$ \\
\hline 33 & $\begin{array}{l}\text { Knothe, G.; Matheaus, A.C.; Ryan III, T.W. (2003). "Cetane Numbers of Branched and } \\
\text { Straight-Chain Fatty Esters Determined in an Ignition Quality Tester." Fuel (82:8); pp. 971- } \\
\text { 975. http://dx.doi.org/10.1016/S0016-2361(02)00382-4 }\end{array}$ \\
\hline 34 & $\begin{array}{l}\text { Knothe, G.; Bagby, M.O.; Ryan III, T.W. (1997). "Cetane Numbers of Fatty Compounds: } \\
\text { Influence of Compound Structure and of Various Potential Cetane Improvers." SAE } \\
\text { Technical Paper 971681. http://dx.doi.org/10.4271/971681 }\end{array}$ \\
\hline 35 & Deleted \\
\hline 36 & $\begin{array}{l}\text { Abou-Rachid, H.; El Marrouni, K.; Kaliaguine, S. (2003). "DFT Studies of the Hydrogen } \\
\text { Abstraction from Primary Alcohols by } \mathrm{O}_{2} \text { in Relation with Cetane Number Data." J. } \\
\text { Molecular Structure: THEOCHEM. }(631: 1-3) \text {; pp. } 241- \\
\text { 250. http://dx.doi.org/10.1016/S0166-1280(03)00257-4 }\end{array}$ \\
\hline 37 & $\begin{array}{l}\text { Abou-Rachid, H.; Bonnevoit, L.; Xu G.; Kaliaguine, S. (2003). "On the Correlation between } \\
\text { Kinetic Rate Constants in the Auto-Ignition Process of Some Oxygenates and Their } \\
\text { Cetane Number: A Quantum Mechanical Study." J. Molecular Structure (621:3) pp. 293- } \\
\text { 304. http://dx.doi.org/10.1016/S0166-1280(02)00676-0 }\end{array}$ \\
\hline 38 & Deleted. \\
\hline 39 & Deleted. \\
\hline 40 & Deleted. \\
\hline 41 & NREL IQT data, most previously unpublished. \\
\hline 42 & $\begin{array}{l}\text { Knothe, G. (2008). “'Designer' Biodiesel; Optimizing Fatty Ester Composition to Improve } \\
\text { Fuel Properties. Energy Fuels 22(2) pp.1358-1364. http://dx.doi.org/10.1021/ef700639e }\end{array}$ \\
\hline 43 & $\begin{array}{l}\text { Moser, B.R.; Knothe, G.; Cermack, S.C. (2010). "Biodiesel from Meadowfoam (Limnanthes } \\
\text { alba L.) Seed Oil: Oxidative Stability and Unusual Fatty Acid Composition." Energy } \\
\text { Environ. Sci. 3(3); pp. 318-327. http://dx.doi.org/10.1039/b923740m }\end{array}$ \\
\hline 44 & $\begin{array}{l}\text { Knothe, G. (2012). "Fuel Properties of Highly Unsaturated Fatty Acid Methyl Esters. } \\
\text { Prediction of Fuel Properties of Algal Biodiesel." Energy Fuels (26:8); pp. 5265- } \\
\text { 5273. http://dx.doi.org/10.1021/ef300700v }\end{array}$ \\
\hline 45 & $\begin{array}{l}\text { Knothe G. (2013). "Fuel properties of Methyl Esters of Borage and Black Currant Oils } \\
\text { Containing Methyl y-linolenate." Eur. J. Lipid Sci. Technol. (115:8); pp. 901- } \\
\text { 908. http://dx.doi.org/10.1002/ej|t.201300061 }\end{array}$ \\
\hline 46 & $\begin{array}{l}\text { Knothe, G. (2014). "A Comprehensive Evaluation of the Cetane Numbers of Fatty Acid } \\
\text { Methyl Esters." Fuel (119); pp. 6-13. http://dx.doi.org/10.1016/j.fuel.2013.11.020 }\end{array}$ \\
\hline 47 & $\begin{array}{l}\text { Boot, M.; Frijters, P.; Luijten, C.; Somers, L.; Baert, R.; Donkerbroek, A.; Klein-Douwel, } \\
\text { R.J.H.; Dam, N. (2009). "Cyclic Oxygenates: A New Class of Second-Generation Biofuels } \\
\text { for Diesel Engines?" Energy and Fuels (23:4); pp. 1808- } \\
\text { 1817. http://dx.doi.org/10.1021/ef8003637 }\end{array}$ \\
\hline 48 & $\begin{array}{l}\text { Hellier, P.; Ladommatos, N.; Allan, R.; Filip, S.; Rogerson, J. (2013). "The Importance of } \\
\text { Double Bond Position and cis-trans Isomerisation in Diesel Combustion and Emissions." } \\
\text { Fuel (105); pp. 477-489. http://dx.doi.org/10.1016/j.fuel.2012.08.007 }\end{array}$ \\
\hline 49 & $\begin{array}{l}\text { Lilik, G.; Boehman, A. (2013). "Effects of Fuel Ignition Quality on Critical Equivalence Ratio } \\
\text { for Autoignition." Energy Fuels (27:3); pp. 1586-1600. http://dx.doi.org/10.1021/ef301600h }\end{array}$ \\
\hline
\end{tabular}




\begin{tabular}{|c|c|}
\hline Number & Reference \\
\hline 50 & $\begin{array}{l}\text { Smagala, T.G., Christensen, E.; Chistison, K.; Mohler, R.E.; Gjersing, E.; McCormick, R.L. } \\
\text { (2013). "Hydrocarbon Renewable and Synthetic Diesel Fuel Blendstocks: Composition and } \\
\text { Properties." Energy Fuels (27:1), pp. 237-246. http://dx.doi.org/0.1021/ef3012849 }\end{array}$ \\
\hline 51 & $\begin{array}{l}\text { Lilik, G.K.; Boehman, A.L. (2013). "Effects of Fuel Composition on Critical Equivalence } \\
\text { Ratio for Autoignition." Energy Fuels, (27:3); pp. 1601- } \\
\text { 1612. http://dx.doi.org/10.1021/ef3016014 }\end{array}$ \\
\hline 52 & $\begin{array}{l}\text { Jenkins, R.W., Munro, M.; Nash, S.; Chuck, C.J. (2013). "Potential Renewable Oxygenated } \\
\text { Biofuels for the Aviation and Road Transport Sectors." Fuel (103); pp. 593- } \\
\text { 599. http://dx.doi.org/10.1016/j.fuel.2012.08.019 }\end{array}$ \\
\hline 53 & $\begin{array}{l}\text { Burger, J.; Siegert, M.; Strofer, E.; Hasse, H. (2010). "Poly(oxymethylene) Dimethyl Ethers } \\
\text { as Components of Tailored Diesel Fuel: Properties, Synthesis and Purification Concepts." } \\
\text { Fuel (89:11); pp. 3315-3319. http://dx.doi.org/10.1016//.fuel.2010.05.014 }\end{array}$ \\
\hline 54 & $\begin{array}{l}\text { Santana, R.C.; Do, P.T.; Santikunaport, M.; Alvarez, W.E.; Taylor, J.D.; Sughrue, E.L.; } \\
\text { Resasco, D.E. (2006). "Evaluation of Different Reaction Strategies for the Improvement of } \\
\text { Cetane Number in Diesel Fuels." Fuel (85:5-6); pp. 643- } \\
\text { 656. http://dx.doi.org/10.1016/j.fuel.2005.08.028 }\end{array}$ \\
\hline 55 & $\begin{array}{l}\text { Heyne, J.S.; Boehman, A.L.; Kirby, S. (2009). "Autoignition Studies of trans- and cis- } \\
\text { Decalin in an Ignition Quality Tester (IQT) and the Development of a High Thermal Stability } \\
\text { Unifuel/Single Battlefield Fuel." Energy Fue/s, (23:12); pp. 5879- } \\
\text { 5885. http://dx.doi.org/10.1021/ef900715m }\end{array}$ \\
\hline 56 & $\begin{array}{l}\text { Bonnhoff, K.; Sausen, E. "Vefahren zur Erhohung der Zundwilligkeit von als Dieselkraftstoff } \\
\text { verwendeten Dialkoxialkanen, insebesondere 1,1-Diethoxyethan." German Patent No. } \\
\text { 3136030, (1981); cited in Schonborn, A.; Hellier, P.; Aliev, A.E.; Ladomatos, N. (2010) } \\
\text { "Ignition Control of Homogeneous-Charge Compression Ignition (HCCl) Combustion } \\
\text { through Adaptation of the Fuel Molecular Structure by Reaction with Ozone." Fuel (89:11); } \\
\text { pp. 3178-3184. http://dx.doi.org/10.1016/j.fuel.2010.06.005 }\end{array}$ \\
\hline 57 & $\begin{array}{l}\text { Haas, F.M.; Ramcharan, A.; Dryer, F.L. (2011). "Relative Reactivities of the Isomeric } \\
\text { Butanols and Ethanol in an Ignition Quality Tester." Energy Fuels (25:9); pp. 3909- } \\
\text { 3916. http://dx.doi.org/10.1021/ef2008024 }\end{array}$ \\
\hline 58 & $\begin{array}{l}\text { Haas, F.M.; Dryer, F.L. (2013). "Prediction of Biofuel Ignition Quality using a DCN } \leftrightarrow \text { RON } \\
\text { Interconversion Tool." In Fall Technical Meeting of the Eastern States Section of the } \\
\text { Combustion Institute, Clemson University, Clemson, SC. }\end{array}$ \\
\hline 59 & $\begin{array}{l}\text { ASTM Standard D6890. (2009). Standard Test Method for Determination of Ignition Delay } \\
\text { and Derived Cetane Number (DCN) of Diesel Fuel Oils by Combustion in a Constant } \\
\text { Volume Chamber. ASTM International: West Conshohocken, PA. }\end{array}$ \\
\hline 60 & $\begin{array}{l}\text { Won, S.H.; Dooley, S.; Veloo, P.S.; Wang, H.; Oehlschlaeger, M.A.; Dryer, F.L.; Ju, Y. } \\
\text { (2014). "The Combustion Properties of 2,6,10-Trimethyl Dodecane and a Chemical } \\
\text { Functional Group Analysis." Combustion and Flame (161:3); pp. 826- } \\
\text { 834. http://dx.doi.org/10.1016/j.combustflame.2013.08.010 }\end{array}$ \\
\hline 61 & $\begin{array}{l}\text { Dooley, S.; Won, S.H.; Jahangirian, S.; Ju, Y.; Dryer, F.L.; Wang, H.; Oehlschlaeger, M.A. } \\
\text { (2012). "The Combustion Kinetics of a Synthetic Paraffinic Jet Aviation Fuel and a } \\
\text { Fundamentally Formulated Experimentally Validated Surrogate Fuel." Combustion and } \\
\text { Flame (159:10); pp. 3014-3020. http://dx.doi.org/10.1016/j.combustflame.2012.04.010 }\end{array}$ \\
\hline 62 & $\begin{array}{l}\text { Dooley, S.; Won, S.H.; Chaos, M.; Heyne, J.; Ju, Y.; Dryer, F.L.; Kumar, K.; Sung, C.-J.; } \\
\text { Wang, H.; Oehlschlaeger, M.A.; Santoro, R.J.; Litzinger, T.A. (2010). "A Jet Fuel Surrogate } \\
\text { Formulated by Real Fuel Properties." Combustion and Flame (157:12); pp. 2333- } \\
\text { 2339. http://dx.doi.org/10.1016/j.combustflame.2010.07.001 }\end{array}$ \\
\hline
\end{tabular}




\begin{tabular}{|c|c|}
\hline Number & Reference \\
\hline 63 & $\begin{array}{l}\text { Dooley, S.; Won, S.H.; Heyne, J.; Farouk, T.I.; Ju, Y.; Dryer, F.L.; Kumar, K.; Hui, X.; } \\
\text { Sung, C.-J.; Wang, H.; Oehlschlaeger, M.A.; Iyer, V.; Iyer, S.; Litzinger, T.A.; Santoro, R.J.; } \\
\text { Malewicki, T.; Brezinsky, K. (2012). "The Experimental Evaluation of a Methodology for } \\
\text { Surrogate Fuel Formulation to Emulate Gas Phase Combustion Kinetic Phenomena." } \\
\text { Combustion and Flame (159:4); pp. 1444- } \\
\text { 1466. http://dx.doi.org/10.1016/j.combustflame.2011.11.002 }\end{array}$ \\
\hline 64 & $\begin{array}{l}\text { Ramcharan, A.M. (2011). Formulation of a Biodiesel Surrogate Fuel through Investigation } \\
\text { of the Ignition Delay Using an Ignition Quality Tester. Undergraduate Thesis. Princeton, } \\
\text { NJ: Princeton University. }\end{array}$ \\
\hline 65 & $\begin{array}{l}\text { Baumgardner, M.E.; Sarathy, S.M.; Marchese, A.J. (2013). "Autoignition Characterization } \\
\text { of Primary Reference Fuels and } n \text {-Heptane/ } n \text {-Butanol Mixtures in a Constant Volume } \\
\text { Combustion Device and Homogeneous Charge Compression Ignition Engine." Energy } \\
\text { Fuels (27:12); pp. 7778-7789. http://dx.doi.org/10.1021/ef4015982 }\end{array}$ \\
\hline 66 & $\begin{array}{l}\text { Szybist, J.P.; McFarlane, J.; Bunting, B. (2007). "Comparison of Simulated and } \\
\text { Experimental Combustion of Biodiesel Blends in a Single Cylinder Diesel HCCI Engine." } \\
\text { SAE 2007-01-4010. http://dx.doi.org/10.4271/2007-01-4010 }\end{array}$ \\
\hline 67 & Anderson, J.E., (2014) personal communication. \\
\hline 68 & $\begin{array}{l}\text { Knothe, G., (2008). " ‘Designer' Biodiesel: Optimizing Fatty Ester Composition to Improve } \\
\text { Fuel Properties." Energy Fuels (22:2); pp. 1358- } \\
\text { 1364. http://pubs.acs.org/doi/abs/10.1021/ef700639e }\end{array}$ \\
\hline 69 & $\begin{array}{l}\text { McCormick, R.L.; Ratcliff, M.A.; Christensen, E.; Fouts, L.; Luecke, J.; Chupka, G.M.; } \\
\text { Yanowitz, J.; Tian, M.; Boot, M. (2015). "Properties of Oxygenates Found in Upgraded } \\
\text { Biomass Pyrolysis Oil as Components of Spark and Compression Ignition Engine Fuels." } \\
\text { Energy Fuels (29:4); pp. 2453-2461. http://dx.doi.org/10.1021/ef502893g. }\end{array}$ \\
\hline 70 & $\begin{array}{l}\text { McCormick, R.L.; Ratcliff, M.; Christensen, E.; Yanowitz, J.; Marchese, A.J.; Olsen, D.; } \\
\text { Vaughn, T.L.; Drenth, A.; Lakshminarayanan, A. (2014). "Impact Of Lignocellulosic } \\
\text { Biomass-Derived Oxygenates on Diesel Fuel Properties and Engine Emissions." SAE } \\
\text { International Powertrain, Fuels and Lubricants Conference, Birmingham, UK, October 22, } \\
2014 .\end{array}$ \\
\hline 71 & $\begin{array}{l}\text { Bönnhoff, K.; Sausen, E. (1981). Verfahren zur Erhöhung der Zündwilligkeit von als } \\
\text { Dieselkraftstoff verwendeten Dialkoxialkanen, insbesondere 1,1-Diethoxyethan. German } \\
\text { Patent No. } 3136030 .\end{array}$ \\
\hline 72 & $\begin{array}{l}\text { Sudholt, A.; Cai, L.; Heyne, J.; Haas, F.M.; Pitsch, H.; Dryer, F.L. (2014). "Ignition } \\
\text { Characteristics of a Bio-Derived Class of Saturated and Unsaturated Furans for Engine } \\
\text { Applications." Proc. Comb. Inst. (35:3); pp.2957- } \\
\text { 2965. http://dx.doi.org/10.1016//.proci.2014.06.147 }\end{array}$ \\
\hline 73 & Pacific Northwest National Laboratory, unpublished. \\
\hline 74 & $\begin{array}{l}\text { Harvey, B.G.; Merriman, W.W.; Koontz, T.A. (2015). "High-Density Renewable Diesel and } \\
\text { Jet Fuels Prepared from Multicyclic Sesquiterpanes and a 1-Hexene-Derived Synthetic } \\
\text { Paraffinic Kerosene." Energy Fuels (29:4); pp. 2431-2436. } \\
\text { http://dx.doi.org/10.1021/ef5027746. }\end{array}$ \\
\hline 75 & $\begin{array}{l}\text { Harvey, B.G.; Harrison, K.W.; Davis, M.C.; Chafin, A.P.; Baca, J.B.; Merriman, W.W. } \\
\text { (2016). "Molecular Design and Characterization of High-Cetane Alkyl Diamondoid Fuels." } \\
\text { Energy Fuels (30:12); pp. 10171- } \\
\text { 10178. http://dx.doi.org/10.1021/acs.energyfuels.6b01865. }\end{array}$ \\
\hline
\end{tabular}




\begin{tabular}{|l|l|}
\hline Number & Reference \\
\hline 76 & $\begin{array}{l}\text { Dahmen, M.; Marquardt, W. (2015). "A Novel Group Contribution Method for the Prediction } \\
\text { of the Derived Cetane Number of Oxygenated Hydrocarbons." Energy Fuels (29:9); pp. } \\
\text { 5781-5801. http://pubs.acs.org/doi/full/10.1021/acs.energyfuels.5b01032 }\end{array}$ \\
\hline 77 & $\begin{array}{l}\text { Jameel, A.G.A.; Naser, N.; Emwas, A.; Dooley, S.; Sarathy, S.M. (2016). "Predicting Fuel } \\
\text { Ignition quality Using 1H NMR Spectroscopy and Multiple Linear Regression." Energy Fuels } \\
\text { (30:11); pp. 9819-9835. http://dx.doi.org/10.1021/acs.energyfuels.6b01690 }\end{array}$ \\
\hline
\end{tabular}




\section{References}

1. For a more complete discussion, see Obert, E.F. (1973). Internal Combustion Engines and Air Pollution. New York: Intext Educational, p. 299ff.

2. Dec, J. (1997). "A Conceptual Model of DI Diesel combustion Based on Laser-Sheet Imaging." SAE Technical Paper 970873: Accessed July 31, 2014:

http://dx.doi.org/10.4271/970873.

3. Yu, Y.C.; Uyehara, O.A.; Myers, P.S.; Collins, R.N.; Mahadevan, K. (1956). "Physical and Chemical Ignition Delay in an Operating Diesel Engine Using the Hot-Motored Technique." SAE Technical Paper 560061. Accessed May 30, 2014: http://dx.doi.org/10.4271/560061

4. Bogin, G.E.; DeFilippo, A.; Chen, J.Y.; Chin, G.; Ratcliff, M.A.; Zigler, B.T.; Dean, A.M. (2011). "Numerical and Experimental Investigation of $n$-Heptane Autoignition in the Ignition Quality Tester (IQT).” Energy Fuels (25:12); pp. 5562-5572. Accessed May 30, 2014: http://dx.doi.org/10.1021/ef201079g

5. Boerlage G.D.; Broeze, J.J. (1933). “Knock Rating for High-Speed C.I. Engine Fuels.” World Petroleum Congress Proceedings, (2); p. 271.

6. J. of Institution of Petroleum Technol. (24); 1938, p. 170, cited in Miner, D.; Seastone, J.B. eds. (1955). Handbook of Engineering Materials. New York: John Wiley and Sons.

7. Falk, K.G. (1906). "The Ignition Temperatures of Hydrogen-Oxygen Mixtures.” J. American Chemical Society (28); p.1517. Accessed May 30, 2014: http://dx.doi.org/10.1021/ja01977a001

8. Dixon H.B.; Howard, H.F. (1909). "Ignition Temperatures of Gases." J. Chem. Soc., (95); p. 514.

9. Mullins, B.P. (1955). Spontaneous Ignition of Liquid Fuels. London, England: Butterworths Scientific Publications. 117 pp.

10. Hurn, R.W.; Hughes, K.J. (1952). "Combustion Characteristics of Diesel Fuels as Measured in a Constant-Volume Combustion Bomb - A Report of the Coordinating Research Council, Inc.” SAE Technical Paper 520210. Accessed May 30, 2014: http://dx.doi.org/10.4271/520210

11. Ryan, T.W. (1985). "Correlation of Physical and Chemical Ignition Delay to Cetane Number.” SAE Technical Paper 852103. Accessed May 30, 2014: http://dx.doi.org/10.4271/852103

12. Ryan, T.W.; Callahan, T.J. (1988). "Engine and Constant Volume Bomb Studies of Diesel Ignition and Combustion." SAE Technical Paper 881626. Accessed May 30, 2014: http://dx.doi.org/10.4271/881626

13. Allard, L.N.; Webster, G.D.; Hole, N.J.; Ryan, T.W.; Ott, D.; Fairbridge, C.W. (1996). "Diesel Fuel Ignition Quality as Determined in the Ignition Quality Tester (IQT)." SAE Technical Paper 961182. Accessed May 30, 2014: http://dx.doi.org/10.4271/961182 
14. Allard, L.N.; Hole, N.J.; Webster, G.D.; Ryan, T.W.; Ott, D.; Beregszazy, A.; Fairbridge, C.W.; Cooley, J.; Mitchell, K.; Richardson, E.K.; Elliot, N.G.; Rickeard, D.J. (1997). "Diesel Fuel Ignition Quality as Determined in the Ignition Quality Tester (IQT) - Part II." SAE Technical Paper 971636. Accessed May 30, 2014: http://dx.doi.org/10.4271/971636

15. Allard, L.N.; Webster, G.D.; Ryan, T.W.; Baker, G.; Beregszaszy, A.; Fairbridge, C.W.; Ecker, A.; Rath, J. (1999). "Analysis of the Ignition Behaviour of the ASTM D-613 Primary Reference Fuels and Full Boiling Range Diesel Fuels in the Ignition Quality Tester (IQT) - Part III." SAE Technical Paper 1999-01-3591. Accessed May 30, 2014:

http://dx.doi.org/10.4271/1999-01-3591

16. Yates, A.D.B.; Viljoen, C.L.; Swarts, A. (2004). "Understanding the Relation between Cetane Number and Combustion Bomb Ignition Delay Measurements." SAE Technical Paper 2004-01-2017. Accessed May 30, 2014: http://dx.doi.org/10.4271/2004-01-2017

17. Serdari, A.; Lois, E.; Stournas, S. (1999). "Impact of Esters of Mono- and Dicarboxylic Acids on Diesel Fuel Quality.” Ind. Eng. Chem. Res. (38:9); pp. 3543-3548. Accessed May 30, 2014: http://dx.doi.org/10.1021/ie9900115

18. Guibet, J.-C. (1999). Fuels and Engines. (1); p. 339.

19. Rose, J.W.; Cooper, J.R., eds. (1977). Technical Data on Fuel, Seventh Edition. New York: Wiley. p. 290.

20. Puckett, A.D.; Caudle, B.H. (July 1948). "Ignition Qualities of Hydrocarbons in the DieselFuel Boiling Range.” United States Bureau of Mines Information Circular 7474,

21. Chevron. (2007). Diesel Fuels Technical Review FTR-2. Accessed May 30, 2014: https://www.google.com/url?q=http://www.chevronwithtechron.ca/products/documents/Diesel_F uel Tech Review.pdf\&sa=U\&ei=eFiGU6GSIcubyATEm4DoDQ\&ved=0CCIQFjABOAo\&usg $=$ AFQjCNEbGC9eSkLXIUSKcYGid5NaGIPK-A

22. Knothe, G.; Matheaus, A.C.; Ryan III, T.W. (2003). "Cetane Numbers of Branched and Straight-Chain Fatty Esters Determined in an Ignition Quality Tester.” Fuel (82:8); 971-975. Accessed May 30, 2014: http://dx.doi.org/10.1016/S0016-2361(02)00382-4

23. Knothe, G.; Bagby, M.O.; Ryan III, T.W. (1997). "Cetane Numbers of Fatty Compounds: Influence of Compound Structure and Various Potential Cetane Improvers." SAE Technical Paper 971681. Accessed May 30, 2014: http://dx.doi.org/10.4271/971681

24. Freedman, B.; Bagby, M.O.; Callahan, T.J.; Ryan, T.W. (1990). "Cetane Numbers of Fatty Esters, Fatty Alcohols and Triglycerides Determined in a Constant Volume Combustion Bomb." SAE Technical Paper 900343. Accessed May 30, 2014: http://dx.doi.org/10.4271/900343

25. Hashimoto, K.; Nakajima, T.; Arai, M; and Tamura, M. (2003). Journal of the Japan Petroleum Institute (46:2); pp. 142-147. 
26. Robbins, W.E.; Audette, R.R.; Reynolds N.E. (1951). "Performance and Stability of Some Diesel Fuel Ignition Quality Improvers." SAE Technical Paper 510200. Accessed May 30, 2014: http://dx.doi.org/10.4271/510200

27. Mueller, C.J.; Cannella, W.J.; Bruno, T.J.; Bunting, B.; Dettman, H.D.; Franz, J.A.; Huber, M.L.; Natarajan, M.; Pitz, W.J.; Ratcliff, M.A.; Wright, K. (2012). "Methodology for Formulating Diesel Surrogate Fuels with Accurate Compositional, Ignition-Quality, and Volatility Characteristics." Energy Fuels (26:6); pp. 3284-3303. Accessed June 26, 2014 : http://pubs.acs.org/doi/abs/10.1021/ef300303e

28. Bowden, J.N.; Johnston, A.A.; Russell, J.A. (1974). “Octane-Cetane Relationship.” Southwest Research Institute, prepared for U.S. Army Mobility Equipment and Development Research and Development Center. Accessed June 26, 2014:

http://www.dtic.mil/dtic/tr/fulltext/u2/779512.pdf

29. Ladommatos, N.; Goacher, J. (1995). "Equations for Predicting the Cetane Number of Diesel Fuels from Their Physical Properties." Fuel (74:7); pp. 1083-1093. Accessed May 30, 2014: http://dx.doi.org/10.1016/0016-2361(95)00040-C

30. Ghosh, P.; Jaffe, S.B. (2006). "Detailed Composition-Based Model for Predicting the Cetane Number of Diesel Fuels." Ind. Eng. Chem. Res. (45:1); 346-351. Accessed May 30, 2014: http://dx.doi.org/10.1021/ie0508132

31. Taylor, J.; McCormick, R.L.; Clark, W. (2004). Report on the Relationship between Molecular Structure and Compression Ignition Fuels, Both Conventional and HCCI. NREL/MP540-36726. Golden, CO: National Renewable Energy Laboratory. Accessed May 30, 2014: http://www.nrel.gov/docx/gen/old/36726.pdf

32. American Society for Testing and Materials. (1958). Knocking Characteristics of Pure Hydrocarbons. ASTM Special Technical Publication No. 225, developed under American Petroleum Institute Research Project 45.

33. Livingston, H.K. (1951). "Knock Resistance of Pure Hydrocarbons.” Ind. Eng. Chem. (43:12); pp. 2834-2840. Accessed May 30, 2014: http://dx.doi.org/10.1021/ie50504a052 